\title{
Aplicação do método do Gradiente Espectral Projetado ao problema de \\ Compressive Sensing
}

\author{
Boris Chullo Llave \\ DissertaÇÃo DE MESTRAdo APRESEnTAdA \\ $\mathrm{AO}$ \\ Instituto De Matemática e Estatística \\ DA \\ Universidade De São Paulo \\ Programa: Ciências da Computação \\ Orientador: Prof. Ernesto G. Birgin
}

Durante o desenvolvimento deste trabalho o autor recebeu auxílio financeiro da CAPES

São Paulo, outubro de 2012 


\section{Aplicação do método do Gradiente Espectral Projetado ao problema de Compressive Sensing}

Esta versão da dissertação contém as correções e alterações sugeridas pela Comissão Julgadora durante a defesa da versão original do trabalho, realizada em 19/09/2012. Uma cópia da versão original está disponível no

Instituto de Matemática e Estatística da Universidade de São Paulo.

Comissão Julgadora:

- Prof. Dr. Ernesto Julian Goldberg Birgin (orientador) - IME-USP

- Prof. Dr. Ronaldo Fumio Hashimoto - IME-USP

- Prof. Dr. Luis Felipe Cesar da Rocha Bueno - UNIFESP 
Dedico este trabalho a Deus que com sua companhia e santa presença foi possivel finalizar este trabalho. Dedico também este trabalho a minha familia, pela paciência e amor, que me motivaram a continuar na luta diária, especialmente nos momentos difíceis. Dedico também este trabalho à mulher que se torno em minha amiga e companheira, sem cujo amor não teria sido possivel finalizar este trabalho: minha noiva Ruth Tito Champi. 


\section{Resumo}

A teoria de Compressive Sensing proporciona uma nova estratégia de aquisição e recuperação de dados com bons resultados na área de processamento de imagens. Esta teoria garante recuperar um sinal com alta probabilidade a partir de uma taxa reduzida de amostragem por debaixo do limite de Nyquist-Shanon. O problema de recuperar o sinal original a partir das amostras consiste em resolver um problema de otimização. O método de Gradiente Espectral Projetado é um método para minimizar funções suaves em conjuntos convexos que tem sido aplicado com frequência ao problema de recuperar o sinal original a partir do sinal amostrado. Este trabalho dedica-se ao estudo da aplicação do Método do Gradiente Espectral Projetado ao problema de Compressive Sensing.

Palavras-chave: Compressive Sensing, Otimização Contínua, Processamento de Imagens, Gradiente Espectral Projetado. 


\section{Abstract}

The theory of compressive sensing has provided a new acquisition strategy and data recovery with good results in the image processing area. This theory guarantees to recover a signal with high probability from a reduced sampling rate below the Nyquist-Shannon limit. The problem of recovering the original signal from the samples consists in solving an optimization problem. The Spectral Projected Gradient (SPG) is a method to minimize continuous functions over convex sets which often has been applied to the problem of recovering the original signal from sampled signals. This work is dedicated to the study and application of the Spectral Projected Gradient method to Compressive Sensing problems.

Keywords: Compressive Sensing, Continuous Optimization, Image Processing, Spectral Projected Gradient. 


\section{Sumário}

1 Introdução 1

2 Visão global de Compressive Sensing 4

2.1 O problema de Compressive Sensing . . . . . . . . . . . . . . . . 4

$2.2 \quad$ A base Wavelet $\Psi \ldots \ldots \ldots \ldots \ldots \ldots \ldots$

2.2.1 A transformada de Haar . . . . . . . . . . . . . . . . . . . . . . . . . . . . . . . . . . . . 11

2.2.2 Funções base da transformada Haar . . . . . . . . . . . . . . . . . 12

2.3 A matriz de medição $\Phi \ldots \ldots \ldots \ldots \ldots \ldots \ldots$

3 Resolução do problema de otimização 18

3.1 Reformulação do problema . . . . . . . . . . . . . . . . . . . 18

3.2 Análise do custo de avaliar a função objetivo e seu gradiente . . . . . . . . . . . . 19

3.3 Gradiente Espectral Projetado . . . . . . . . . . . . . . . . . . 25

4 Experimentos computacionais $\quad 30$

4.1 Experimentos preliminares . . . . . . . . . . . . . . . . . . . . 30

4.2 Experimentos sem ruido . . . . . . . . . . . . . . . . . . . . 33

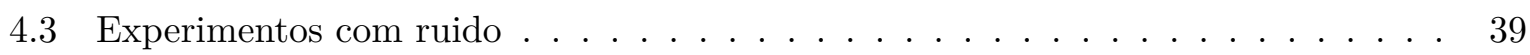

4.4 Experimentos de grande porte . . . . . . . . . . . . . . . . 48

$\begin{array}{llr}5 & \text { Conclusões } & 61\end{array}$

$\begin{array}{ll}\text { Referências Bibliográficas } & 63\end{array}$ 


\section{Capítulo 1}

\section{Introdução}

A aquisição e reconstrução de sinais é um ramo essencial no estudo de sistemas de processamento de sinais. O teorema de Nyquist-Shannon garante que é possível recuperar exatamente um sinal sempre que o sinal seja de banda limitada e a taxa de amostragem seja pelo menos duas vezes a maior frequência do espectro do sinal [38]. Este teorema, desde sua formulação, tem sido usado como ferramenta para estabelecer as frequências de amostragem mínimas de sinais analógicos. Recentemente, uma nova teoria chamada Compressive Sensing (CS), também conhecida como Compressed Sensing ou Compressive Sampling, foi proposta como um paradigma eficiente de aquisição e reconstrução de dados.

A teoria de Compressive Sensing foi desenvolvida inicialmente por Emmanuel Candès em [18] e Donoho em [51] e assegura que um sinal pode ser recuperado dado um número pequeno de medições lineares aleatórias. Isto significa que é possível reconstruir um sinal $x \in \mathbb{R}^{n} k$-esparso no domínio $\Psi$, mediante $m$ medições lineares $y=\Phi x$, em que $m \gtrsim k \log n$. Comparado com a teoria tradicional de amostragem de Nyquist-Shannon, o CS provê uma grande redução na taxa de amostragem, consumo de energia e complexidade computacional na aquisição e representação de um sinal esparso.

Compressive Sensing é um campo que tem sido impulsado largamente por avanços teóricos e práticos que fornecem tanto a motivação como a direção para as aplicações. Dentre as aplicações que envolvem o uso de Compressive Sensing na aquisição e recuperação de sinais podemos mencionar: a câmera de um pixel, ressonância magnética em imagens médicas e prospecção geofísica ou sismologia, dentre outras.

O processo de amostragem pode ser interpretado como produtos internos do sinal a considerar $x \in \mathbb{R}^{n}$ com uma coleção de vetores de teste $\left\{\phi_{j}\right\}_{j=1}^{m}$. Esta interpretação fornece uma ideia prática para fazer a amostragem dos dados sem a aquisição completa do sinal $x$. A câmera de um pixel [30] foi desenvolvida na Universidade de Rice [42] e é o dispositivo mais conhecido em Compressive Sensing. Para fazer a amostragem, este dispositivo usa um "computador óptico" e é composta de um dispositivo micro-espelho digital (DMD), duas lentes, um detector de fóton e um conversor analógico-digital [30]. O DMD consiste de um arranjo de $n$ micro-espelhos, cada um dos quais corresponde a um pixel em $x$. Cada micro-espelho é configurado de forma a refletir 
a luz em direção ao único pixel de recebimento, ou em alguma outra direção. Por sua vez, a lente foca a luz para o detector, que lê o produto interno (como uma tensão) entre a configuração atual dos micro-espelhos (um vector de uns e zeros) e os pixels de $x$. Esta tensão é digitalizada e o processo é repetido para $m$ diferentes configurações aleatórias dos espelhos. A configuração do sistema permite dar valores de \pm 1 nas entradas de cada $\phi_{i}$ e assim, obter as $m$ amostras $y=\Phi x$. Considerando que a imagem é compressível numa base adequada, pode-se então obter uma aproximação da imagem original usando um algoritmo de CS. Além dos benefícios usuais de CS, esta arquitetura permite o uso de vários tipos de detectores [30], incluindo espectrômetros e fotodiodos que são, por exemplo, sensíveis a diferentes comprimentos de onda. Uma vez que o custo de um arranjo de $m$ detectores pode ser elevado, o uso deste aplicativo restringe-se a situações nas quais o custo do sensoreamento é excessivamente alto.

$\mathrm{Na}$ área de imagens médicas, em especial, na Ressonância Magnética (MR), em que se medem os coeficientes de Fourier das imagens, a teoria de CS encontra uma aplicação importante. As imagens de MR são implicitamente esparsas. Algumas imagens de MR, tais como angiogramas, são esparsas em seu próprio domínio de representação (base canônica), enquanto outras imagens de MR mais complexas são esparsas em outro domínio como, por exemplo, as wavelet. Como sabemos, o processo de Ressonância Magnética em geral é muito custoso no que se refere ao tempo de aquisição das imagens. Assim, seria muito útil reduzir o tempo de amostragem sem sacrificar a qualidade da imagem. Em [41], explora-se a esparsidade das imagens de MR para, juntamente com CS, conseguir reduzir significativamente o tempo de aquisição dos dados.

Os dados sísmicos são coletados geralmente em tabelas multidimensionais e envolvem informação acima dos Terabytes. De forma simplificada, o processo de aquisição é realizado como segue. No processo de aquisição utilizam-se várias fontes colocadas na superfície da terra. Estas fontes transmitem energia através do subsolo. Subsequentemente, a resposta de cada receptor é recolhida e armazenada. Motivados pela teoria de CS, Hennenfent e Herrmann [37] propuseram um paradigma alternativo de aquisição que consiste na utilização de um número reduzido de fontes e receptores colocados numa grade subamostrada aleatoriamente perturbada. Observando-se que os dados sísmicos são esparsos, por exemplo, no domínio curvelet [37], a recuperação do sinal pode-se modelar como um problema de CS.

O CS baseia-se nos conceitos de "esparsidade" e "incoerência", que são propriedades do sinal de interesse e da matriz de medição, respectivamente. A esparsidade indica o grau em que a informação contida no sinal pode ser concisamente representada em uma base $\Psi$ escolhida adequadamente. Em outras palavras, o numero de coeficientes não nulos no domínio da base fornece uma medida da compressibilidade do sinal. A incoerência, por outro lado, proporciona uma medida do grau de semelhança entre a matriz de medição $\Phi$ e a base $\Psi$. Para alcançar um elevado grau de precisão na reconstrução de um sinal, um esquema de CS requer que o sinal de interesse seja muito esparso no domínio $\Psi$ e que a matriz de medição seja o mais incoerente possível com relação à matriz da base esparsa $\Psi$.

Infelizmente, encontrar $\Psi$ e $\Phi$ adequadas não é uma tarefa trivial. Por um lado, a natureza 
de $\Phi$ esta ligada fortemente à construção de dispositivos de aquisição, quer dizer, o desenho da matriz de aquisição $\Phi$ deve ser simples. Por outro lado, a coerência com relação à matriz base esparsa $\Psi$ deve ser muito baixa. O modelo de matriz de medição que utilizaremos neste texto é descrito em [52], onde brevemente são estudadas as matrizes de medição existentes e propõe-se um novo desenho altamente incoerente com as muitas bases conhecidas. Após feita a amostragem, algoritmos de reconstrução devem ser aplicados para recuperar o sinal original a partir das amostras. Esta reconstrução baseia-se na resolução de um problema de otimização. $\mathrm{O}$ método do Gradiente Espectral Projetado (SPG) introduzido em $[12,16]$ é um método utilizado para minimizar funções suaves em conjuntos convexos. Neste trabalho de mestrado estudaremos a aplicação do SPG à versão ponderada do problema de Compressive Sensing considerada em $[33]$.

Este trabalho esta organizado como segue. No Capítulo 2 introduziremos formalmente o problema de Compressive Sensing e estudaremos o problema de otimização que consiste em recuperar o sinal original a partir das amostras. No Capítulo 3 analisaremos em detalhe o problema de otimização e a forma de resolução usando o SPG. No Capítulo 4 apresentaremos e analisaremos experimentos computacionais. Conclusões finais serão apresentadas no Capítulo 5.

\section{Notação:}

Dado $x \in \mathbb{R}^{n}$, definimos a norma $\ell_{p}$ de $x \in \mathbb{R}^{n}$ como:

$$
\|x\|_{p}=\left(\sum_{j=1}^{n}\left|x_{j}\right|^{p}\right)^{1 / p}, 0<p<\infty .
$$

Em particular, para $p=1,2, \infty$, temos que:

$$
\|x\|_{1}=\sum_{i=1}^{n}\left|x_{i}\right|, \quad\|x\|_{2}=\left(\sum_{i=1}^{n} x_{i}^{2}\right)^{1 / 2}, \quad\|x\|_{\infty}=\max _{i=1, \ldots, n}\left|x_{i}\right| .
$$

Seja um subconjunto de índices $T \subseteq\{1, \ldots, n\}$, denotamos por $x_{T} \in \mathbb{R}^{n}$ o vetor com entradas $x_{i}$ para cada $i \in T$, e zero para os índices fora de $T$. Similarmente, se $A \in \mathbb{R}^{n \times n}$ então $A_{T} \in$ $\mathbb{R}^{n \times|T|}$ denota as colunas de $A$ correspondentes aos índices em $T$, em que $|T|$ é a cardinalidade de $T . T^{c}=\{1, \ldots, n\} \backslash T$ denota o complemento de $T$. O núcleo da matriz $A$ é denotado como $\mathcal{N}(A)=\left\{x \in \mathbb{R}^{n} \mid A x=0\right\}$.

Uma base de um espaço vetorial $\mathbb{V}$ é um conjunto de vetores em $\mathbb{V}$ linearmente independentes que geram esse espaço. Se os vetores de uma base $\mathbb{V}$ são ortogonais entre si e tem norma Euclidiana unitária, dizemos que $\mathbb{V}$ é uma base ortonormal. 


\section{Capítulo 2}

\section{Visão global de Compressive Sensing}

Atualmente, muitas técnicas de compressão de imagens como JPG e JPG2000 [27] baseiam-se em estratégias adaptativas de recolha de informação. Estes algoritmos constroem uma representação esparsa completa de um sinal amostrado, para depois armazenar os coeficientes mais significativos ${ }^{1}$, definindo assim um sinal menor ou um sinal comprimido.

Em essência, a teoria de CS é motivada pela seguinte questão citada por Donoho [51]: "Por quê fazer tanto esforço para adquirir todos os dados quando a maior parte deles será descartada? Não podemos simplesmente medir diretamente a parte que não será descartada?". Se fossem conhecidas a priori as posições dos coeficientes mais significativos, teríamos a imagem comprimida medindo estes coeficientes e ignorando a informação restante. No entanto, por um lado não conhecemos a informação inteira do sinal, e muito menos as posições dos coeficientes significativos, e, por outro lado contamos apenas com um esquema de medição não adaptativo no sentido de que o mesmo esquema é utilizado no procedimento de captura de sinais diversos. Assim, CS é um paradigma de aquisição de dados que tenta realizar simultaneamente o processo de amostragem e a redução de dimensionalidade, no pressuposto de que o sinal é esparso.

Neste capítulo apresentamos formalmente o problema de Compressive Sensing e apresentamos exemplos de bases esparsas e matrizes de medição. O capítulo termina com a formulação do problema de otimização relacionado à recuperação da imagem original a partir das amostras.

\subsection{O problema de Compressive Sensing}

Nesta seção apresentamos a formulação matemática do problema de Compressive Sensing e as definições formais de algumas terminologias que serão utilizadas mas adiante.

Seja um sinal $x \in \mathbb{R}^{n}$ e uma base ortonormal $\left\{\psi_{1}, \psi_{2}, \cdots, \psi_{n}\right\}$ de $\mathbb{R}^{n}$, tal que a representação do sinal na base é dada por:

$$
x=\sum_{i=1}^{n} s_{i} \psi_{i}
$$

\footnotetext{
${ }^{1}$ Esta etapa é chamada codificação e tem como objetivo mapear os coeficientes significativos na menor sequência de bits possível.
} 
onde $s=\left(s_{1}, s_{2}, \ldots, s_{n}\right)^{T}$ é o vetor de coeficientes da expansão de $x$. Se denotamos $\Psi=\left[\psi_{1} \psi_{2} \cdots \psi_{n}\right]^{T}$ temos

$$
x=\Psi^{T} s .
$$

Como $\Psi$ é uma matrix ortogonal segue-se que $s=\Psi x$. Substituindo $s$ em (2.2) temos que

$$
x=\Psi^{T} \Psi x=\sum_{i=1}^{n}\left(\psi_{i}^{T} x\right) \psi_{i} .
$$

Quer dizer, temos $s_{i}=\left\langle\psi_{i}, x\right\rangle$. Se $s$ é esparso então é possível descartar os coeficientes menos significativos de $s$ sem ter uma perda perceptível no sinal.

Formalmente, dado $p$, definimos o conjunto $S_{p}$, formado pelos índices dos $p$ coeficientes de maior módulo em $s$ e consideramos $\hat{x}=\Psi^{T} s_{S_{p}}$ ou $s_{S_{p}}=\Psi \hat{x}$. Nestas condições, o vetor $s_{S_{p}}$ é chamado $\left|S_{p}\right|$-esparso dado que tem no máximo $\left|S_{p}\right|$ elementos diferentes de zero. Uma vez que $\Psi$ é uma matriz ortogonal, temos $\|x-\hat{x}\|_{2}=\|\Psi x-\Psi \hat{x}\|_{2}=\left\|s-s_{S_{p}}\right\|_{2}$ e, se $s_{S_{p}}$ é uma boa aproximação esparsa de $s$, no sentido de que todas as componentes significativas de $s$ fazem parte de $s_{S_{p}}$ então $\left\|s-s_{S_{p}}\right\|_{2}$ é pequena e, portanto, $\hat{x}$ é uma boa aproximação de $x$ [20].

Mais genericamente, o nível de esparsidade de um sinal indica quão aproximado pode ser o sinal resultante após a reconstrução. Determina também quão reduzido pode ser o número de amostras necessárias para recuperar o sinal.

Seja um sinal $x \in \mathbb{R}^{n} k$-esparso no domínio $\Psi$. Quer dizer, $x=\Psi^{T} s$ e $s$ é $k$-esparso (i.e. tem no máximo $k$ componentes não nulas). O esquema de aquisição consiste em reunir amostras a partir de produtos internos do sinal $x$ com vetores de teste $\left\{\phi_{i}\right\}_{i=1}^{m}$, em que $m \ll n$ (veja a Figura 2.1). Assim, adquirimos $m$ medições $y_{i}=\left\langle\phi_{i}, x\right\rangle, i \in\{1, \ldots, m\}$, isto é

$$
y_{1}=\left\langle\phi_{1}, x\right\rangle, y_{2}=\left\langle\phi_{2}, x\right\rangle, \ldots, y_{m}=\left\langle\phi_{m}, x\right\rangle
$$

Em notação matricial, podemos representá-lo sucintamente como $y=\Phi x$, onde a matriz de medição $\Phi$ é composta de linhas $\phi_{i}^{T}$ e $y \in \mathbb{R}^{m}$. Logo, temos que

$$
y=\Phi x=\Phi \Psi^{T} s=\Theta s,
$$

onde $\Theta=\Phi \Psi^{T}, \Theta \in \mathbb{R}^{m \times n}$, com $m \ll n$ e $m \gtrsim k \log n$. Veja a Figura 2.2 .

Dado que o número de equações é menor que o numero de variáveis, existem infinitas soluções que satisfazem (2.5). Neste ponto a esparsidade joga um papel muito importante já que, dentre todas estas soluções, procuramos a solução mais esparsa. Mesmo nos restringindo a soluções $k$-esparsas, não é possível garantir que a solução será única. A unicidade pode ser conseguida se a matriz de medição $\Phi$ e a matriz da base $\Psi$ forem incoerentes entre si [17].

Apenas como exemplo, note que se $\phi_{i}=(1, \ldots, 1)^{T}, i=1, \ldots, m$, então teríamos $y_{i}=\sum_{j=1}^{n} x_{j}, i=$ 


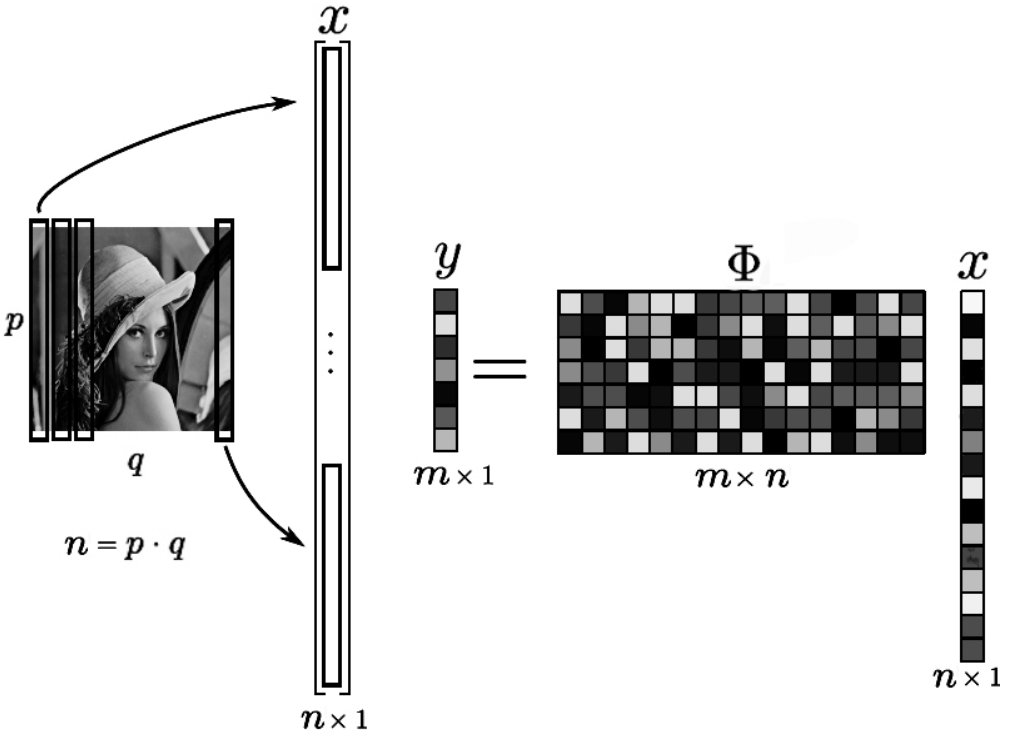

Figura 2.1: Vectorização de uma imagem e extração das m medições lineares (extraída de [48]).

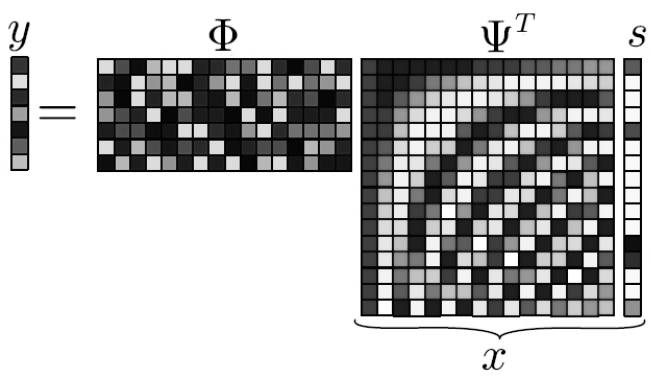

(a)

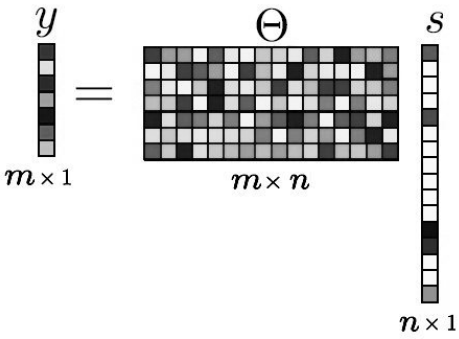

(b)

Figura 2.2: (a) Representação esparsa do sinal $x$ no domínio $\Psi$ (as entradas claras em $s$ representam coeficientes pouco significativos) e extração das amostras y de $x$ utilizando a matriz de amostragem $\Phi$. (b) Representação gráfica do problema geral (veja que $\Theta=\Phi \Psi^{T}$ ) (extraída de [48]).

$1, \ldots, m$, e seria impossível recuperar o sinal $x$ dessas $m$ medições idênticas.

A propriedade de isometria restrita é um conceito introduzido em [21] que demonstrou ser muito útil na teoria geral de CS. Como será mostrado posteriormente, este conceito fornece uma ferramenta muito conveniente para determinar condições suficientes que garantam a reconstrução exata do sinal esparso para diferentes algoritmos de reconstrução.

Definição 1 [21]: Seja $\Theta \in \mathbb{R}^{m \times n}$ e $1 \leq k \leq n$. Diz-se que $\Theta$ satisfaz a $k$-ésima propriedade de isometria restrita se existe uma constante $\delta_{k}$ tal que

$$
\left(1-\delta_{k}\right)\|s\|_{2}^{2} \leq\|\Theta s\|_{2}^{2} \leq\left(1+\delta_{k}\right)\|s\|_{2}^{2},
$$

para todo $s k$-esparso, $s \in \mathbb{R}^{n}$.

Se uma matriz $\Theta$ satisfaz a $k$-ésima propriedade de isometria restrita com $\delta_{k}$ "pequeno" então 
$\Theta$ comporta-se quase como uma matriz ortogonal quando enxergada como uma transformação linear aplicada a vetores $k$-esparsos.

O seguinte exemplo, apresentado em [21], reflete a conexão entre a propriedade de isometria restrita e a teoria de CS. Suponha que desejamos recuperar um sinal $p$-esparso com $p \leq k$ a partir de $m$ amostras feitas no sinal. Isto é, procuramos $s^{\prime}$ o mais esparso possível que satisfaça $\Theta s^{\prime}=y$, o que nos leva a formular o problema conhecido como minimização $\ell_{0}$, dado por

$$
\min \|s\|_{0} \text { sujeita a } \Theta s=y,
$$

onde $\|s\|_{0}=\#\left\{i \in\{1, \ldots, n\} \mid s_{i} \neq 0\right\}$.

É possível ver que se a matriz $\Theta$ satisfizer a $2 k$-ésima propriedade de isometria restrita com constante $\delta_{2 k}<1$ então o problema (2.7) terá solução $p$-esparsa com $p \leq k$ única. Suponha por contradição que existem duas soluções diferentes $p$-esparsas $s_{1}$ e $s_{2}$ com $p \leq k$ tais que $\Theta s_{1}=\Theta s_{2}=y$. Consideremos $z=s_{1}-s_{2}$, que, claramente, tem no máximo $2 k$ entradas diferentes de zero, isto é, o vetor $z$ é $2 k$-esparso. Logo

$$
\Theta z=\Theta\left(s_{1}-s_{2}\right)=\Theta s_{1}-\Theta s_{2}=0
$$

De (2.8) segue que $\|\Theta z\|_{2}^{2}=0$. Logo, da Definição 1, segue-se que

$$
\left(1-\delta_{2 k}\right)\|z\|_{2}^{2} \leq\|\Theta z\|_{2}^{2}=0
$$

De $(2.9)$ temos que $\left(1-\delta_{2 k}\right) \leq 0$, pois $\|z\|_{2}^{2}>0$ já que $z=s_{1}-s_{2}$ e $s_{1} \neq s_{2}$ por hipótese, contradizendo a hipótese de que $\delta_{2 k}<1$.

As constantes de isometria restrita são difíceis de calcular [5] e referem-se à matriz $\Theta=\Phi \Psi^{T}$. Veremos agora uma propriedade que relaciona a matriz de medição $\Phi$ e a matriz da base esparsa $\Psi$.

Definição 2 [29]: Sejam $\Phi=\left[\phi_{1}, \ldots, \phi_{m}\right]^{T} \in \mathbb{R}^{m \times n}$ e $\Psi=\left[\psi_{1}, \ldots, \psi_{n}\right]^{T} \in \mathbb{R}^{n \times n}$ tais que $\left\|\phi_{i}\right\|_{2}=1, i=1, \ldots, m$ e $\left\|\psi_{j}\right\|_{2}=1, j=1, \ldots, n$. Definimos a coerência entre $\Phi$ e $\Psi$ como

$$
\mu(\Phi, \Psi)=\sqrt{n} \max _{1 \leq i \leq m, 1 \leq j \leq n}\left\{\left|\left\langle\phi_{i}, \psi_{j}\right\rangle\right|\right\}
$$

Uma propriedade muito importante da matriz de medição é a incoerência, introduzida por Donoho e Huo em [29]. Este conceito pede que a matriz de medição $\Phi$ seja tal que, dada a matriz da base esparsa $\Psi, \mu(\Phi, \Psi)$ em (2.10) seja o menor possível. Isto é, se, por exemplo, a matriz de medição $\Phi$ fosse formada por $m$ linhas da base esparsa $\Psi$ selecionadas aleatoriamente, então um grande número de coeficientes nulos seria amostrado e não haveria informação suficiente para reconstruir o sinal. No entanto, se $\Phi$ e $\Psi$ são incoerentes, no sentido de que não existe relação entre as linhas de $\Phi$ e as colunas de $\Psi$, uma grande informação será adquirida do sinal. Assim, 
em cada medição, aprende-se algo novo sobre o sinal.

Se $\Phi$ e $\Psi$ contêm elementos correlacionados, a coerência entre elas será grande. Caso contrário, será pequena. É possível ver que $\mu(\Phi, \Psi) \in[1, \sqrt{n}]$. No exemplo do parágrafo anterior, $\mu(\Phi, \Psi)=$ $\sqrt{n}$ (máxima coerência). Da definição (2.10) e a desigualdade Cauchy-Schwarz temos que

$$
\mu(\Phi, \Psi)=\sqrt{n} \max _{1 \leq i \leq m, 1 \leq j \leq n}\left\{\left|\left\langle\phi_{i}, \psi_{j}\right\rangle\right|\right\} \leq \sqrt{n} \max _{1 \leq i \leq m, 1 \leq j \leq n}\left\{\left\|\phi_{i}\right\|\left\|\psi_{j}\right\|\right\}=\sqrt{n} .
$$

Por outro lado, da ortonormalidade da matriz $\Psi$, temos que

$$
\sum_{j=1}^{n}\left(\left|\left\langle\phi_{i}, \psi_{j}\right\rangle\right|\right)^{2}=\left\|\Psi^{T} \phi_{i}\right\|_{2}^{2}=\left\|\phi_{i}\right\|_{2}^{2}=1, \text { para } i=1, \ldots, m .
$$

$\sum_{j=1}^{n}\left(\left|\left\langle\phi_{i}, \psi_{j}\right\rangle\right|\right)^{2}=1$ para $i=1, \ldots, m$ implica que, dado $1 \leq i \leq m$, existe $1 \leq j \leq n$ tal que $\left|\left\langle\phi_{i}, \psi_{j}\right\rangle\right| \geq 1 / \sqrt{n}$. Logo, $\max _{1 \leq i \leq m, 1 \leq j \leq n}\left\{\left|\left\langle\phi_{i}, \psi_{j}\right\rangle\right|\right\} \geq 1 / \sqrt{n}$ e, portanto, $\mu(\Phi, \Psi) \geq 1$. Concluímos que $\mu(\Phi, \Psi) \in[1, \sqrt{n}]$ como queriamos mostrar.

Dados $\Phi$ e $\Psi$, calcular a coerência $\mu(\Phi, \Psi)$ é uma tarefa simples que envolve calcular o máximo entre $m n$ produtos internos. Por outro lado verificar se um valor dado corresponde à $k$-ésima contante de isometria restrita da matriz $\Theta=\Phi \Psi^{T}$ é uma tarefa difícil que envolve verificar (2.6) para todos os possíveis $\left(\begin{array}{l}n \\ k\end{array}\right)$ subconjuntos de $k$ colunas da matriz $\Theta$. Mesmo que isso fosse possível, ainda restaria verificar que a constante dada é a menor dentre todas as que satisfazem (2.6) já que, casso isso não fosse feito, teríamos apenas um limitante superior da $k$-ésima constante de isometria restrita da matriz $\Theta$. Em [7] foi mostrada uma relação entre a coerência das matrizes $\Phi$ e $\Psi$ e as constantes de isometria restrita da matriz $\Theta=\Phi \Psi^{T}$ :

$$
\delta_{k} \leq(k-1) \mu(\Phi, \Psi) / \sqrt{n}
$$

Logo, se baseando apenas na incoerência das matrizes $\Phi$ e $\Psi$ é possível determinar se a matriz $\Theta=\Phi \Psi^{T}$ é uma matriz adequada à formulação do problema de CS ou não.

Infelizmente resolver o problema de minimização $\ell_{0}$ (2.7) é NP-difícil [35]. Porém, abordagens mais eficientes do ponto de vista computacional cujas soluções podem ser interpretadas como aproximações da solução de (2.7) são a minimização $\ell_{1}$ e a minimização $\ell_{2}$, que serão abordadas a seguir.

O problema de minimização de norma $\ell_{2}$ consiste em

$$
\min \|s\|_{2} \text { sujeita a } \Theta s=y,
$$

que é equivalente a resolver

$$
\min \frac{1}{2}\|s\|_{2}^{2} \text { sujeita a } \Theta s=y
$$


As condições KKT para o problema (2.13) são:

$$
\begin{aligned}
s+\Theta^{T} \lambda & =0, \\
\Theta s & =y .
\end{aligned}
$$

Isolando $s$ de (2.14) e substituindo em (2.15) temos

$$
-\Theta \Theta^{T} \lambda=y .
$$

Se $\Theta \in \mathbb{R}^{m \times n}$, com $m \leq n$, tiver posto completo, então temos que $\Theta \Theta^{T}$ é não singular e de (2.16) segue-se que

$$
\lambda=-\left(\Theta \Theta^{T}\right)^{-1} y .
$$

Substituindo (2.17) em (2.14) resulta

$$
s^{*}=\Theta^{T}\left(\Theta \Theta^{T}\right)^{-1} y .
$$

Infelizmente esta solução nem sempre fornece soluções esparsas.

O problema de minimização de norma $\ell_{1}$ consiste em

$$
\min \|s\|_{1} \text { sujeita a } \Theta s=y \text {. }
$$

O problema (2.19) é conhecido como problema basis pursuit (BP) [22]. Surpreendentemente, este "relaxamento" muitas vezes recupera o sinal original desde que o sinal seja esparso no domínio $\Psi$. Note-se que a matriz $\Theta$ é dada e fixada antecipadamente e não depende do sinal. Porém, se o sinal for esparso e as matrizes $\Phi$ e $\Psi$ forem incoerentes a minimização da norma $\ell_{1}$ fornecera uma boa aproximação da solução do problema (2.7) [18] (veja a Figura 2.3).

Em aplicações reais, as amostras podem conter erros nas medições, isto é

$$
y=\Theta s+\eta,
$$

onde $\eta$ é o erro derivado das medições. Neste contexto, procuramos um $s$ que minimize o resíduo $\|y-\Theta s\|_{2}^{2}$ do sistema linear $\Theta s=y$ e, além disso, seja esparso. Então, nosso objetivo agora consiste em minimizar simultaneamente $\|s\|_{1}$ e $\|y-\Theta s\|_{2}^{2}$. Isto está dado pelo problema:

$$
\min _{s \in \mathbb{R}^{n}}\left(\|s\|_{1},\|y-\Theta s\|_{2}^{2}\right) .
$$

O problema (2.21) é um tipo de problema de otimização com dois objetivos, conhecido como problema de otimização multiobjetivo [44]. Para uma descrição detalhada do problema de otimização multiobjetivo e as diferentes técnicas de resolução veja, por exemplo, [47].

Se o nível de ruido nos dados é conhecido ou pode ser estimado, o problema (2.21) pode ser 


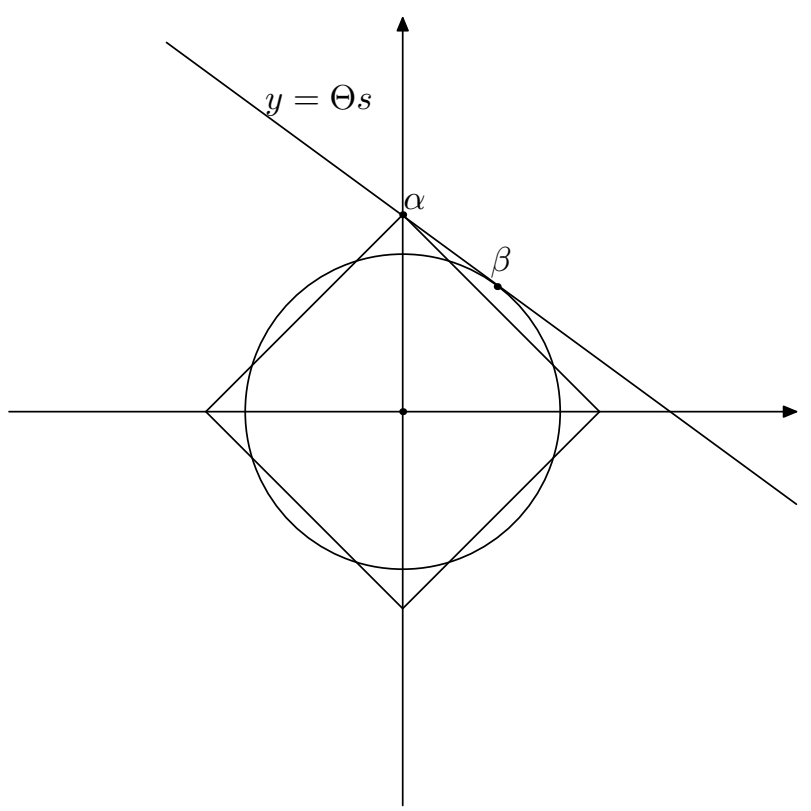

Figura 2.3: $\alpha=\operatorname{argmin}\|s\|_{1}$ sujeita a $\Theta s=y$ e $\beta=\operatorname{argmin}\|s\|_{2}$ sujeita a $\Theta s=y$. Note que $\alpha_{2}=0$ enquanto que $\beta_{1} \neq 0$ e $\beta_{2} \neq 0$. De alguma forma o gráfico sugere que a minimização da norma $\ell_{1}$ resulta ter soluções mais esparsas do que a minimização da norma $\ell_{2}$.

reduzido a resolver um problema da forma

$$
\min \|s\|_{1} \text { sujeita a }\|y-\Theta s\|_{2}^{2} \leq \epsilon,
$$

conhecido como problema basis pursuit denoise $\left(\mathrm{BP}_{\varepsilon}\right)$, onde o parâmetro $\varepsilon>0$ é uma estimativa do nível de ruido nos dados. Claramente, se $\varepsilon=0$, a solução do problema $\mathrm{BP}_{\varepsilon}$ corresponde com a solução do problema BP.

O problema $\left(\mathrm{BP}_{\varepsilon}\right)$ (2.22) é uma das formas possíveis de atacar o problema (2.21). Outras formas possíveis incluem o problema de quadrados mínimos penalizado $\left(\mathrm{QP}_{\gamma}\right)$ [22] dado por

$$
\min _{s \in \mathbb{R}^{n}}\|y-\Theta s\|_{2}^{2}+\gamma\|s\|_{1}
$$

e o problema Lasso $\left(\mathrm{LS}_{\tau}\right)[50]$ dado por

$$
\min _{s \in \mathbb{R}^{n}}\|y-\Theta s\|_{2}^{2} \text { sujeita a }\|s\|_{1} \leq \tau .
$$

Para valores apropriados dos parâmetros $\varepsilon, \gamma$ e $\tau$, as soluções dos problemas $\mathrm{BP}_{\varepsilon}, \mathrm{QP}_{\gamma}$ e $\mathrm{LS}_{\tau}$ coincidem e, portanto, estes três problemas são de alguma forma equivalentes [31].

Em [31] aborda-se o problema $\mathrm{BP}_{\varepsilon}$. O algoritmo proposto baseia-se no fato de que o problema $\mathrm{BP}_{\varepsilon}$ pode ser resolvido aplicando o método do Gradiente Espectral Projetado a uma sequência de problemas $\mathrm{LS}_{\tau}$. A aplicação eficiente do SPG ao problema $\mathrm{LS}_{\tau}$ depende de forma crucial de um algoritmo com complexidade $O(n \log n)$ para calcular a projeção no conjunto convexo dado por $\|s\| \leq \tau$. Em [33], o problema $\mathrm{QP}_{\lambda}$ é abordado. A técnica utilizada consiste em 
reformular o problema $\mathrm{QP}_{\lambda}$ (2.23) como um problema contínuo e diferenciável de minimizar uma função quadrática sujeita a restrições de caixa e resolve-lo utilizando o método SPG. Neste trabalho, seguindo a metodologia descrita em [33], consideraremos a resolução de uma sequência de problemas da forma (2.23).

\section{$2.2 \quad$ A base Wavelet $\Psi$}

As transformadas de wavelets podem ser vistas como mecanismos para decompor ou quebrar sinais nas suas partes constituintes, permitindo analisar os dados em diferentes domínios de frequências com a resolução de cada componente associada à sua escala.

As wavelets mais usadas (Haar [36] e Daubechies [43]) formam um sistema ortonormal de funções com suportes compactos construídos a partir de dilatações e translações. Ou seja, mediante as dilatações, elas podem distinguir as características locais de um sinal em diferentes escalas. Por outro lado, pelas translações, elas cobrem toda a região na qual o sinal é analisado. Se pudéssemos escolher os coeficientes das wavelets que melhor se adaptam aos dados, ou ignorássemos os coeficientes menores do que um valor previamente estabelecido, os dados poderiam ser esparsamente representados. Esta codificação esparsa faz das wavelets uma excelente ferramenta no campo de compressão de dados. Nas próximas subseções daremos ênfase à análise de sinais com wavelets de Haar.

\subsubsection{A transformada de Haar}

Nesta subseção discutiremos a descomposição de um sinal usando wavelets de Haar. Descreveremos ainda como é que essa descomposição, que chamaremos daqui em diante Transformada de Haar, pode levar a uma técnica simples de compressão de sinais.

Para ter uma noção de como funcionam as wavelets, começamos com um exemplo bem simples. Suponha que temos uma imagem unidimensional com uma resolução de 4 pixels, tendo os seguintes valores

$$
\left[\begin{array}{llll}
8 & 6 & 5 & 7
\end{array}\right] \text {. }
$$

Podemos representar essa imagem na base de Haar, calculando uma transformada wavelet. Para fazer isto, calculamos a média aritmética para pares de pixels consecutivos na imagem. Este resultado será denominado coeficiente médio. No exemplo, os coeficientes médios da imagem são:

$$
\left[\begin{array}{ll}
7 & 6
\end{array}\right]
$$

já que $\frac{1}{2}(8+6)=7$ e $\frac{1}{2}(5+7)=6$ Claramente notamos que alguma informação foi perdida neste processo. Para recuperar a imagem completa, precisamos armazenar informação adicional, que chamaremos coeficientes de detalhe, e armazenarão a informação desperdiçada. Em nosso exemplo, temos 1 como nosso primeiro coeficiente de detalhe, dado que o primeiro coeficiente médio computado é 1 menos do que 8 (primeiro pixel da imagem original) e 1 mais do que 6 (segundo pixel da imagem original). Este número apenas permite-nos recuperar os primeiros 
dois pixels da imagem original de um total de 4 pixels. Similarmente, o segundo coeficiente de detalhe é -1 , visto que $6+(-1)=5$ e $6-(-1)=7$.

Assim, decompomos a imagem original em uma imagem com uma resolução mais baixa. Repetindo o processo iterativamente sobre os coeficientes médios calculados temos a descomposição completa:

\begin{tabular}{|c|c|c|}
\hline Resolução & Média & Detalhe \\
\hline 4 & {$\left[\begin{array}{llll}8 & 6 & 5 & 7\end{array}\right]$} & \\
\hline 2 & {$\left[\begin{array}{ll}7 & 6\end{array}\right]$} & {$\left[\begin{array}{ll}1 & -1\end{array}\right]$} \\
\hline 1 & {$[6.5]$} & {$[0.5]$} \\
\hline
\end{tabular}

Finalmente, a transformada wavelet (chamada também decomposição wavelet) da imagem original de 4 pixels é formada pelo único coeficiente que representa a média global da imagem original, seguido pelos coeficientes de detalhe, que permitem recuperar a resolução original. Assim, a transformada wavelet da imagem original é dada por

$$
\left[\begin{array}{llll}
6.5 & 0.5 & 1 & -1
\end{array}\right] .
$$

Armazenar os coeficientes da imagem transformada, ao invés da imagem em si, trás uma série de vantagens. Uma vantagem da transformada wavelet é que muitas vezes muitos dos coeficientes de detalhe acabam por ser muito pequenos em magnitude. Truncar ou ignorar esses coeficientes pequenos, pode apresentar erros desprezíveis na imagem reconstruída, dando uma representação comprimida da imagem com pouca perda de informação.

\subsubsection{Funções base da transformada Haar}

Nesta subseção analisaremos em detalhe as funções que compõem a transformada de Haar, a imagem e sua representação esparsa.

Alternativamente, trataremos as imagens como funções com valores constantes no intervalo $[0,1)$. Uma imagem de um pixel é uma função com valor constante em todo o intervalo [0,1). O espaço vetorial para estas funções será definido como $V_{0}$. Assim, o espaço $V_{1}$ contém todas as imagens de dois pixels, que tomam valores constantes nos intervalos $[0,1 / 2)$ e $[1 / 2,1)$ e o espaço $V_{2}$ incluirá todas as imagens de quatro pixels, constantes nos intervalos $[0,1 / 4),[1 / 4,1 / 2),[1 / 2,3 / 4)$ e $[3 / 4,1)$. Generalizando, o espaço $V_{j}$ incluirá todas as imagens de $2^{j}$ pixels, constantes em $2^{j}$ subintervalos iguais do intervalo $[0,1)$.

Podemos pensar em cada imagem unidimensional com $2^{j}$ pixels como um elemento, ou como um vetor de $V_{j}$. Note que estas funções são definidas em um intervalo unitário, então todos os vetores em $V_{j}$ também estão contidos em $V_{j+1}$. Isto é,

$$
V_{0} \subset V_{1} \subset V_{2} \subset \ldots
$$


Precisamos definir uma base para cada espaço vetorial $V_{j}$. As funções base para os espaços $V_{j}$ são chamadas funções escala, e são denotadas usualmente como $\phi$. A base para $V_{j}$ é dada pelo conjunto de funções escala:

$$
\phi_{j, k}(x)=\phi\left(2^{j} x-k\right), \quad k=0, \ldots, 2^{j}-1,
$$

em que

$$
\phi(x)= \begin{cases}1, & x \in[0,1), \\ 0, & \text { caso contrário. }\end{cases}
$$

Como exemplo, as Figuras 2.4(a-b) ilustram as duas e as quatro funções base para os espaços $V_{1}$ e $V_{2}$, respectivamente.
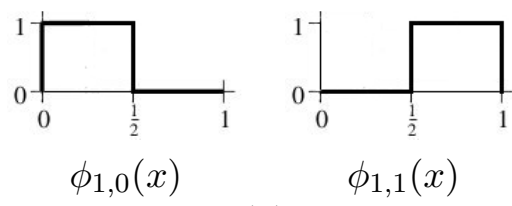

(a)
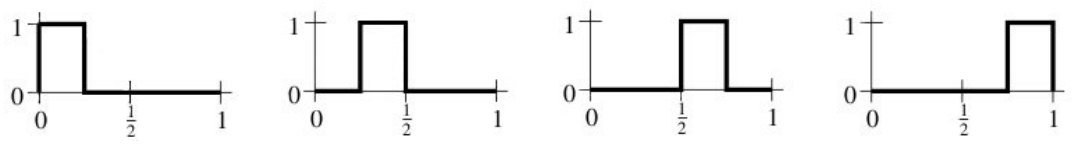

$$
\phi_{2,0}(x)
$$

$$
\phi_{2,1}(x)
$$

(b)

$$
\phi_{2,3}(x)
$$

Figura 2.4: (a) Funções base de $V_{1}$. (b) Funções base de $V_{2}$

Definimos um novo espaço vetorial $W_{j}$ como o complemento ortogonal de $V_{j}$ em $V_{j+1}$. Em outras palavras, $W_{j}$ será o espaço das funções em $V_{j+1}$ ortogonais a todas as funções em $V_{j}$. Isto é,

$$
V_{j+1}=V_{j} \oplus W_{j}
$$

Definimos a função wavelet dada por:

$$
\psi_{j, k}(x)=\psi\left(2^{j} x-k\right), k=0, \ldots, 2^{j}-1,
$$

onde $\psi$ no caso da wavelet de Haar é definida como:

$$
\psi(x)=\left\{\begin{array}{rc}
1, & x \in[0,1 / 2), \\
-1, & x \in[1 / 2,1), \\
0, & \text { caso contrário. }
\end{array}\right.
$$

Em [26] mostra-se que o conjunto $\left\{\psi_{j, k}(x)\right\}_{k=0, \ldots, 2^{j}-1}$ forma uma base para o espaço $W_{j}$. As funções $\psi$ e $\phi$ possuem duas propriedades importantes:

1. As funções base $\psi_{j, k}(x)$ do espaço $W_{j}$, sempre formam, com as funções base $\phi_{j, k}(x)$ do espaço $V_{j}$, uma base para o espaço $V_{j+1}[26]$. 
2. Todas as funções base $\psi_{j, k}(x)$ do espaço $W_{j}$ são ortogonais a todas as funções base $\phi_{j, k}(x)$ do espaço $V_{j}[26]$.

Assim, notamos que os coeficientes de detalhe da Seção 2.2.1 são de fato os coeficientes da base de funções wavelet. As Figura 2.5(a-b) mostram as duas e as quatro wavelets de Haar qie geram o espaço $W_{1}$ e $W_{2}$, respectivamente.

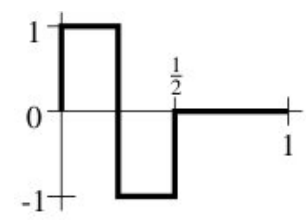

$\psi_{1,0}(x)$

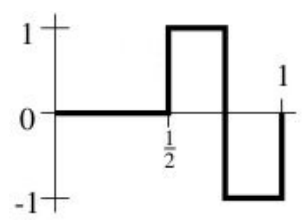

$\psi_{1,1}(x)$

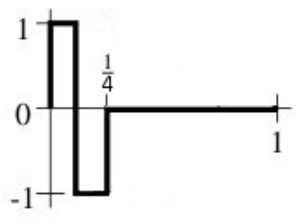

$\psi_{2,0}(x)$

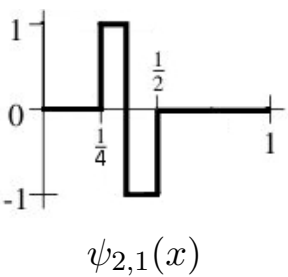

(a)

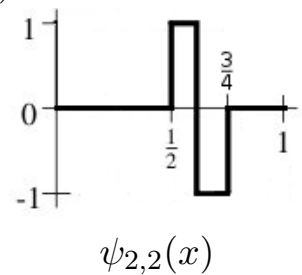

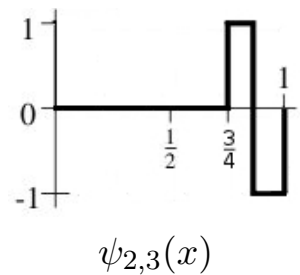

$\psi_{2,3}(x)$

(b)

Figura 2.5: (a) As wavelets de Haar para o espaço $W_{1}$. (b) As wavelets de Haar para o espaço $W_{2}$.

Denotemos por $\mathfrak{I}(x)$ a imagem de 4 pixels do exemplo da Seção 2.2.1. Então $\mathfrak{I}(x)$ pode ser escrita como combinação linear dos elementos da base de $V_{2}$ :

$$
\mathfrak{I}(x)=c_{2,0} \phi_{2,0}(x)+c_{2,1} \phi_{2,1}(x)+c_{2,2} \phi_{2,2}(x)+c_{2,3} \phi_{2,3}(x),
$$

$\operatorname{com} c_{2,0}=8, c_{2,1}=6, c_{2,2}=5$ e $c_{2,3}=7$.

Mostramos uma versão gráfica da representação:

$$
\begin{array}{rlll}
\mathfrak{I}(x) & = & \times \sqrt{\square} \\
& +6 & \times \sqrt{\square} \\
& +5 & \times \sqrt{\square} \\
& +
\end{array}
$$

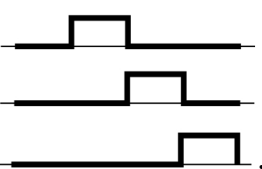

Note que os coeficientes $c_{2,0}, \ldots, c_{2,3}$ são exatamente os 4 pixels da imagem $\left[\begin{array}{llll}8 & 6 & 5 & 7\end{array}\right]$. Podemos reescrever a expressão para $\mathfrak{I}(x)$ em termos das bases de $V_{1}$ e $W_{1}$ já que, em virtude de (2.25), $V_{2}=V_{1} \oplus W_{1}$.

$$
\mathfrak{I}(x)=c_{1,0} \phi_{1,0}(x)+c_{1,1} \phi_{1,1}(x)+d_{1,0} \psi_{1,0}(x)+d_{1,1} \psi_{1,1}(x),
$$

com $c_{1,0}=7, c_{1,1}=6, d_{1,0}=1$ e $d_{1,1}=-1$, ou, graficamente, 


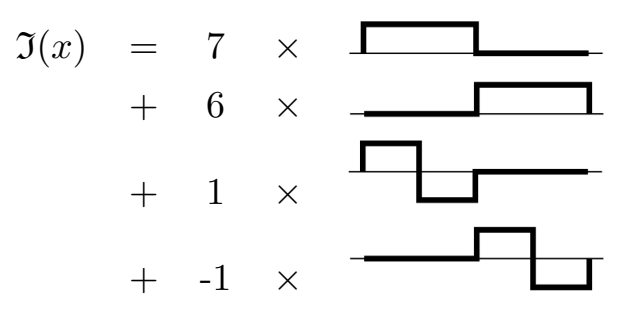

Finalmente, reescrevemos $\mathfrak{I}(x)$ como uma soma das bases de $V_{0}, W_{0}$, e $W_{1}$ :

$$
\mathfrak{I}(x)=c_{0,0} \phi_{0,0}(x)+d_{0,0} \psi_{0,0}(x)+d_{1,0} \psi_{1,0}(x)+d_{1,1} \psi_{1,1}(x),
$$

com $c_{0,0}=6.5, d_{0,0}=0.5, d_{1,0}=1$ e $d_{1,1}=-1$, ou, graficamente,

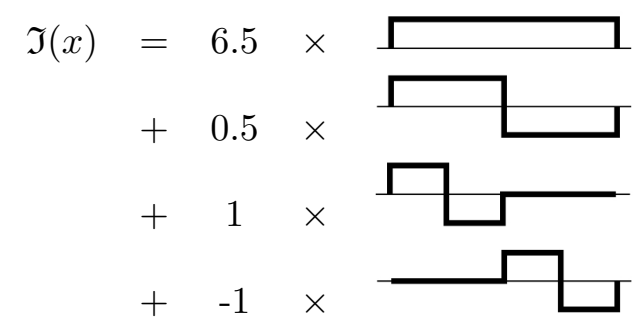

Os coeficientes $\left[\begin{array}{llll}6.5 & 0.5 & 1 & -1\end{array}\right]$ constituem a transformada de Haar da imagem original. As 4 funções $\phi_{0,0}(x), \psi_{0,0}(x), \psi_{1,0}(x)$ e $\psi_{1,1}(x)$ constituem a base Haar para $V_{2}$. Generalizando, em virtude de (2.25). Temos

$$
\begin{aligned}
V_{j} & =V_{j-1} \oplus W_{j-1}, \\
V_{j} & =V_{j-2} \oplus W_{j-2} \oplus W_{j-1}, \\
\vdots & \\
V_{j} & =V_{0} \oplus W_{0} \oplus \cdots \oplus W_{j-1} .
\end{aligned}
$$

Isto é, as funções base dos espaços $V_{0}, W_{0}, \ldots, W_{j-1}$ formam uma base para o espaço $V_{j}$. Esta base é a base Haar para $V_{j}$. Em [26] mostra-se que os vetores da base Haar são ortogonais. Podemos normalizar a base Haar substituindo as definições anteriores por

$$
\begin{aligned}
& \phi_{j, k}(x)=2^{j / 2} \phi\left(2^{j} x-k\right), \\
& \psi_{j, k}(x)=2^{j / 2} \psi\left(2^{j} x-k\right),
\end{aligned}
$$

onde o fator $2^{j / 2}$ é escolhido apenas para satisfazer a normalidade. Assim, no exemplo anterior, os coeficientes da transformada de Haar na base ortonormal de $V_{2}$ são

$$
\left[\begin{array}{llll}
13 & 1 & \sqrt{2} & -\sqrt{2}
\end{array}\right]
$$

Em CS, a base $\Psi$ expressa-se como uma matriz ortogonal. Para sinais $x \in \mathbb{R}^{4}$ a matriz base esparsa $\Psi \in \mathbb{R}^{4 \times 4}$, é dada por $\Psi=H_{2} H_{1}$ com $H_{1}$ e $H_{2}$ dadas pelas seguintes matrizes: 


$$
H_{1}=\left[\begin{array}{cccc}
\frac{1}{\sqrt{2}} & \frac{1}{\sqrt{2}} & 0 & 0 \\
0 & 0 & \frac{1}{\sqrt{2}} & \frac{1}{\sqrt{2}} \\
\frac{1}{\sqrt{2}} & -\frac{1}{\sqrt{2}} & 0 & 0 \\
0 & 0 & \frac{1}{\sqrt{2}} & -\frac{1}{\sqrt{2}}
\end{array}\right] \quad H_{2}=\left[\begin{array}{cccc}
\frac{1}{\sqrt{2}} & \frac{1}{\sqrt{2}} & 0 & 0 \\
\frac{1}{\sqrt{2}} & -\frac{1}{\sqrt{2}} & 0 & 0 \\
0 & 0 & 1 & 0 \\
0 & 0 & 0 & 1
\end{array}\right]
$$

onde ambas matrizes $H_{1}$ e $H_{2}$ definem as etapas da transformada de Haar para o sinal $x$. $\mathrm{O}$ número de etapas vem dado pelo logaritmo na base 2 da dimensão do sinal a tratar, neste caso é $\log _{2} 4=2$. Logo, a matriz $\Psi$ é

$$
\Psi=\left[\begin{array}{l}
\phi_{0,0} \\
\psi_{0,0} \\
\psi_{1,0} \\
\psi_{1,1}
\end{array}\right]=\left[\begin{array}{cccc}
\frac{1}{2} & \frac{1}{2} & \frac{1}{2} & \frac{1}{2} \\
\frac{1}{2} & \frac{1}{2} & -\frac{1}{2} & -\frac{1}{2} \\
\frac{1}{\sqrt{2}} & -\frac{1}{\sqrt{2}} & 0 & 0 \\
0 & 0 & \frac{1}{\sqrt{2}} & -\frac{1}{\sqrt{2}}
\end{array}\right] .
$$

Notamos que as linhas da matriz $\Psi$ são as funções da base Haar, quer dizer $\left\{\phi_{0,0}, \psi_{0,0}, \psi_{1,0}, \psi_{1,1}\right\}$.

\subsection{A matriz de medição $\Phi$}

Um dos problemas mais recorrentes na teoria de CS é o desenho da matriz de medição. A matriz de medição $\Phi$ deve permitir a reconstrução do sinal $x \in \mathbb{R}^{n}$ a partir de $m \gtrsim k \log n$ medições lineares $y=\Phi x$. Na literatura existem muitos esquemas de amostragem. De acordo a sua complexidade podemos classifica-las como segue: (i) Matrizes aleatórias: Matrizes densas onde os elementos são independentes e identicamente distribuídos, por exemplo, matrizes aleatórias gaussianas [51], (ii) Matrizes não-binárias ortogonais: Estas matrizes são o resultado de selecionar um subconjunto de linhas da matriz da transformada de Fourier cujas colunas foram previamente permutadas, por exemplo, Scramble Fourier Matrix (SF) [19], (iii) Matrizes binárias ortogonais: Matrizes que usam funções Noiselet [24] como funções de teste, um exemplo destas matrizes é Partial Noiselet Matrix (PN) [17], (iv) Matrizes esparsas bloco diagonais: Por exemplo, Scramble Block Hadamard (SBH) [28].

Infelizmente nem todas estas matrizes são simples. O termo simples refere-se a que as matrizes não são esparsas e binárias, o que representa uma baixa eficiência de sensoreamento e dificuldade na implementação de hardware. Por conta disso, um novo desenho de matriz de aquisição foi proposto por Zaixing et al. [52] chamado Binary Permuted Block Diagonal Matrix (BPBD). Na seguinte subseção analisaremos o desenho desta matriz.

Dado $L \leq m$, consideremos $L$ submatrizes bloco diagonais $\hat{\mathbf{A}}_{1}=\operatorname{diag}\left(\mathbf{a}_{1}^{1}, \mathbf{a}_{2}^{1}, \ldots, \mathbf{a}_{m_{1}}^{1}\right) \in \mathbb{R}^{m_{1} \times n}$, $\hat{\mathbf{A}}_{2}=\operatorname{diag}\left(\mathbf{a}_{1}^{2}, \mathbf{a}_{2}^{2}, \ldots, \mathbf{a}_{m_{2}}^{2}\right) \in \mathbb{R}^{m_{2} \times n}, \ldots, \hat{\mathbf{A}}_{L}=\operatorname{diag}\left(\mathbf{a}_{1}^{L}, \mathbf{a}_{2}^{L}, \ldots, \mathbf{a}_{m_{L}}^{L}\right) \in \mathbb{R}^{m_{L} \times n} \operatorname{com} m_{1}+m_{2}+$ $\cdots+m_{L}=m$ e blocos $\mathbf{a}_{j}^{i} \in \mathbb{R}^{1 \times k}$ com $k \in\left\{\left\lfloor\frac{n L}{m}\right\rfloor,\left\lfloor\frac{n L}{m}\right\rfloor+1\right\}$. Consideramos também $L$ matrizes de permutação $\mathbf{P}_{1}, \mathbf{P}_{2}, \ldots, \mathbf{P}_{L}$, com $\mathbf{P}_{i} \in \mathbb{R}^{n \times n}$. Construímos a Permuted Block Diagonal Matrix (PBD) $\Phi \in \mathbb{R}^{m \times n}$ permutando as matrizes bloco diagonais com suas respectivas matrizes de 
permutação, quer dizer,

$$
\Phi=\left[\begin{array}{c}
\mathbf{A}_{1} \\
\mathbf{A}_{2} \\
\vdots \\
\mathbf{A}_{L}
\end{array}\right]=\left[\begin{array}{c}
\hat{\mathbf{A}}_{1} \mathbf{P}_{1} \\
\hat{\mathbf{A}}_{2} \mathbf{P}_{2} \\
\vdots \\
\hat{\mathbf{A}}_{L} \mathbf{P}_{L}
\end{array}\right]=\left[\begin{array}{c}
\operatorname{diag}\left(\mathbf{a}_{1}^{1}, \ldots, \mathbf{a}_{m_{1}}^{1}\right) \times \mathbf{P}_{1} \\
\operatorname{diag}\left(\mathbf{a}_{1}^{2}, \ldots, \mathbf{a}_{m_{2}}^{2}\right) \times \mathbf{P}_{2} \\
\vdots \\
\operatorname{diag}\left(\mathbf{a}_{1}^{L}, \ldots, \mathbf{a}_{m_{L}}^{L}\right) \times \mathbf{P}_{L}
\end{array}\right]
$$

As permutações aleatórias são usadas apenas para produzir incoerência com a matriz da base esparsa $\Psi$. As matrizes $\mathbf{A}_{1}, \mathbf{A}_{2}, \ldots, \mathbf{A}_{L}$ são chamadas submatrizes de $\Phi$. A matriz PBD com somente entradas binárias é chamada BPBD. Um exemplo da matriz BPBD é dado por:

$$
\left.\left.\left[\begin{array}{cccccccccc}
1 & 1 & 1 & & & & & & & \\
& & & 1 & 1 & 1 & & & & \\
& & & & & & \ddots & & & \\
& & & & & & & 1 & 1 & 1
\end{array}\right) \times \mathbf{P}_{1}\right] \times \mathbf{P}_{2}\right]
$$

onde $L=2$ e $\mathbf{a}_{j}^{i}=\left[\begin{array}{lll}1 & 1 & 1\end{array}\right], j=1, \ldots, m_{i}$ e $i=1, \ldots, L$.

Um dos grandes aportes da estrutura da matriz BPBD é a facilidade de implementação a nível de hardware e a alta eficiência de sensoreamento. Além disso, uma vez que existem $k$ '1's em cada linha da matriz BPBD, quando amostramos o sinal $x \in \mathbb{R}^{n}$, apenas $k$ elementos de $x$ são considerados. Assim, podemos obter $n / k$ amostras por vez e, realizando $L$ vezes o mesmo processo, todas as $m$ amostras são obtidas. Em muitos algoritmos de reconstrução, por exemplo, Orthonormal Matching Pursuit (OMP)[8] e Tree Matching Pursuit (TMP)[40], são considerados apenas produtos matriz por vetor, que são computados economicamente, dado que a matriz de medição é armazenada de forma implícita. A matriz BPBD tem os requisitos mínimos de armazenamento e fornece uma forma rápida e econômica de fazer o produto matriz-vetor.

Em [52] mostra-se que a incoerência da BPBD com qualquer base esparsa é $O(\sqrt{k})$. Estes resultados obtidos indicam a eficiência do sensoreamento desta matriz. Como a matriz é binária, a implementação é menos custosa e mais tratável. 


\section{Capítulo 3}

\section{Resolução do problema de otimização}

Neste capítulo, seguindo o conteúdo de [33], analisaremos o problema de otimização que consiste em recuperar um sinal esparso a partir de um conjunto de amostras. Inicialmente, apresentaremos uma reformulação contínua e diferenciável do problema (2.23) e analisaremos o custo computacional da avaliação da função objetivo e as derivadas do problema reformulado. Concluiremos o capítulo descrevendo brevemente o método do Gradiente Espectral Projetado.

\subsection{Reformulação do problema}

O problema considerado é basicamente o problema (2.23) e consiste em

$$
\min _{s \in \mathbb{R}^{n}} \gamma\|s\|_{1}+\frac{(1-\gamma)}{2}\|y-\Theta s\|_{2}^{2},
$$

onde:

- $n$ representa o tamanho do sinal $x \in \mathbb{R}^{n}$ que deve ser recuperado,

- $\Psi \in \mathbb{R}^{n \times n}$ é a matriz cujas linhas formam uma base na qual $x$ tem representação esparsa, quer dizer, $\Psi^{T} s=x$ e $s \in \mathbb{R}^{n}$ tem muitos elementos nulos,

- $m$ é número de amostras,

- $\Phi \in \mathbb{R}^{m \times n}$ é a matriz de medição,

- $y=\Phi x \in \mathbb{R}^{m}$ é o vetor de amostras,

- $\Theta=\Phi \Psi^{T}$ e

- $\gamma \in[0,1]$ é um parâmetro dado.

O problema (3.1) é um problema de minimização irrestrita com função objetivo não diferenciável o que impede a sua resolução utilizando algoritmos clássicos de programação não linear que requerem diferenciabilidade da função objetivo. Usando a mudança de variáveis $s=u-v$, com 
$u, v \geq 0$, o problema (3.1) pode ser reescrito como

$$
\min \gamma\left[\sum_{i=1}^{n} u_{i}+\sum_{i=1}^{n} v_{i}\right]+\frac{(1-\gamma)}{2}\|y-\Theta(u-v)\|_{2}^{2} \text { sujeita a } u, v \geq 0
$$

O problema (3.2) consiste em minimizar uma quadrática sujeita a restrições de caixa (não negatividade) nas variáveis. Em formato matricial, o problema (3.2) pode ser reescrito como

$$
\min \frac{(1-\gamma)}{2} z^{T} B z+c^{T} z \text { sujeita a } z \geq 0
$$

onde $z=(u, v)^{T}, 1_{2 n}=[1,1, \ldots, 1]^{T} \in \mathbb{R}^{2 n}$,

$$
B=\left[\begin{array}{rr}
\Theta^{T} \Theta & -\Theta^{T} \Theta \\
-\Theta^{T} \Theta & \Theta^{T} \Theta
\end{array}\right] \text { e } c=\gamma 1_{2 n}+(1-\gamma)\left[\begin{array}{r}
-\Theta^{T} y \\
\Theta^{T} y
\end{array}\right]
$$

Observamos que, a dimensão do problema (3.3) é duas vezes maior do que a dimensão do problema (3.1): $s \in \mathbb{R}^{n}$ enquanto $z \in \mathbb{R}^{2 n}$. No entanto, este incremento não é de grande impacto devido a que o custo de calcular a função objetivo de (3.3) está diretamente relacionado a custo do produto da matriz $B$ por um vetor arbitrário $z$. Este produto pode ser realizado de forma econômica como segue:

$$
B z=B\left[\begin{array}{l}
u \\
v
\end{array}\right]=\left[\begin{array}{rr}
\Theta^{T} \Theta & -\Theta^{T} \Theta \\
-\Theta^{T} \Theta & \Theta^{T} \Theta
\end{array}\right]\left[\begin{array}{l}
u \\
v
\end{array}\right]=\left[\begin{array}{r}
\Theta^{T} \Theta(u-v) \\
-\Theta^{T} \Theta(u-v)
\end{array}\right]=\left[\begin{array}{r}
w \\
-w
\end{array}\right],
$$

onde $w=\Theta^{T} \Theta(u-v)$. Quer dizer, o produto $B z$ pode ser encontrado calculando o vetor diferença $u-v \in \mathbb{R}^{n}$ e, em seguida, multiplicando-o por $\Theta$ e depois por $\Theta^{T}$, com $\Theta \in \mathbb{R}^{m \times n}$. Poder-se-ia concluir que, como o custo de calcular a função objetivo de (3.1) envolve apenas um único produto pela matriz $\Theta$, o preço a pagar por trabalhar com a reformulação diferenciável (3.3) seria dobrar o custo da avaliação da função objetivo. Este preço poderia ser considerado modesto diante da vantagem de poder trabalhar com um problema diferenciável. Porém, como veremos em seguida, o custo de avaliar a função de (3.3) é de fato idêntico ao custo de avaliar a função de (3.1).

\subsection{Análise do custo de avaliar a função objetivo e seu gradiente}

A função objetivo de (3.3) está definida como

$$
F(z)=\frac{(1-\gamma)}{2} z^{T} B z+c^{T} z
$$

Analisando primeiro o produto $z^{T} B z$, temos

$$
z^{T} B z=\left[\begin{array}{ll}
u^{T} & v^{T}
\end{array}\right]\left[\begin{array}{rr}
\Theta^{T} \Theta & -\Theta^{T} \Theta \\
-\Theta^{T} \Theta & \Theta^{T} \Theta
\end{array}\right]\left[\begin{array}{l}
u \\
v
\end{array}\right] .
$$


Substituindo (3.5) em (3.7), temos

$$
z^{T} B z=\left[\begin{array}{ll}
u^{T} & v^{T}
\end{array}\right]\left[\begin{array}{r}
\Theta^{T} \Theta(u-v) \\
-\Theta^{T} \Theta(u-v)
\end{array}\right]=(u-v)^{T} \Theta^{T} \Theta(u-v)=\|\Theta(u-v)\|_{2}^{2} .
$$

Concluímos então que, efetivamente, o cálculo da função objetivo $F(z)$ de (3.3), requer apenas de uma única multiplicação pela matriz $\Theta \in \mathbb{R}^{m \times n}$. Supondo que o vetor constante $c$ definido em (3.4), que depende de $\Theta^{T} y$, é pré-computado e armazenado no início do método, concluímos que custo de avaliar a função objetivo $F(z)$ de (3.3) é equivalente ao custo de avaliar a função objetivo não diferenciável da formulação original (3.1).

Para completar a análise do custo computacional da avaliação objetivo, precisamos analisar o custo do produto matriz-vetor $\Theta(u-v)=\Phi \Psi^{T}(u-v)$. Para isso mostramos a seguir as regras de formação das matrizes $\Phi$ e $\Psi^{T}$, onde $\Phi$ é a matriz de amostragem diagonal por blocos e $\Psi$ é a matriz da transformada de Haar definidas no Capítulo 2. Já que $u-v \in \mathbb{R}^{n}$, faremos a análise considerando $s=u-v$.

Começaremos pela matriz $\Psi^{T}$ analisando o produto $x=\Psi^{T} s$ e, para simplificar a tarefa, analisaremos um exemplo com $n=8=2^{3}, s \in \mathbb{R}^{n}, \Psi \in \mathbb{R}^{n \times n}, \Psi^{T}=\left(H_{3} H_{2} H_{1}\right)^{T}$ com $H_{1}, H_{2}$ e $H_{3}$ dadas por

$$
\begin{gathered}
H_{1}=\left(\begin{array}{cccccccc}
\frac{1}{\sqrt{2}} & \frac{1}{\sqrt{2}} & 0 & 0 & 0 & 0 & 0 & 0 \\
0 & 0 & \frac{1}{\sqrt{2}} & \frac{1}{\sqrt{2}} & 0 & 0 & 0 & 0 \\
0 & 0 & 0 & 0 & \frac{1}{\sqrt{2}} & \frac{1}{\sqrt{2}} & 0 & 0 \\
0 & 0 & 0 & 0 & 0 & 0 & \frac{1}{\sqrt{2}} & \frac{1}{\sqrt{2}} \\
\frac{1}{\sqrt{2}} & -\frac{1}{\sqrt{2}} & 0 & 0 & 0 & 0 & 0 & 0 \\
0 & 0 & \frac{1}{\sqrt{2}} & -\frac{1}{\sqrt{2}} & 0 & 0 & 0 & 0 \\
0 & 0 & 0 & 0 & \frac{1}{\sqrt{2}} & -\frac{1}{\sqrt{2}} & 0 & 0 \\
0 & 0 & 0 & 0 & 0 & 0 & \frac{1}{\sqrt{2}} & -\frac{1}{\sqrt{2}}
\end{array}\right), \\
H_{2}=\left(\begin{array}{cccccccc}
\frac{1}{\sqrt{2}} & \frac{1}{\sqrt{2}} & 0 & 0 & 0 & 0 & 0 & 0 \\
0 & 0 & \frac{1}{\sqrt{2}} & \frac{1}{\sqrt{2}} & 0 & 0 & 0 & 0 \\
\frac{1}{\sqrt{2}} & -\frac{1}{\sqrt{2}} & 0 & 0 & 0 & 0 & 0 & 0 \\
0 & 0 & \frac{1}{\sqrt{2}} & -\frac{1}{\sqrt{2}} & 0 & 0 & 0 & 0 \\
0 & 0 & 0 & 0 & 1 & 0 & 0 & 0 \\
0 & 0 & 0 & 0 & 0 & 1 & 0 & 0 \\
0 & 0 & 0 & 0 & 0 & 0 & 1 & 0 \\
0 & 0 & 0 & 0 & 0 & 0 & 0 & 1
\end{array}\right)
\end{gathered}
$$


$\mathrm{e}$

$$
H_{3}=\left(\begin{array}{cccccccc}
\frac{1}{\sqrt{2}} & \frac{1}{\sqrt{2}} & 0 & 0 & 0 & 0 & 0 & 0 \\
\frac{1}{\sqrt{2}} & -\frac{1}{\sqrt{2}} & 0 & 0 & 0 & 0 & 0 & 0 \\
0 & 0 & 1 & 0 & 0 & 0 & 0 & 0 \\
0 & 0 & 0 & 1 & 0 & 0 & 0 & 0 \\
0 & 0 & 0 & 0 & 1 & 0 & 0 & 0 \\
0 & 0 & 0 & 0 & 0 & 1 & 0 & 0 \\
0 & 0 & 0 & 0 & 0 & 0 & 1 & 0 \\
0 & 0 & 0 & 0 & 0 & 0 & 0 & 1
\end{array}\right)
$$

Como queremos calcular $x=\Psi^{T} s$, calculamos $x=\left(H_{3} H_{2} H_{1}\right)^{T} s=H_{1}^{T}\left(H_{2}^{T}\left(H_{3}^{T} s\right)\right)$. A regra de formação das matrizes $H_{i}$ permite que cada um desses produtos matriz-vetor sejam calculados eficientemente sem sequer precisar construir as matrizes $H_{i}$. O Algoritmo 1 implementa o produto $x=\Psi^{T} s$ e o produto $s=\Psi x$ (que será necessário no cálculo do gradiente da função objetivo), dependendo do valor do parâmetro de entrada work. Quando work $=T$, o Algoritmo 1 recebe como parâmetro de entrada $\lambda \in \mathbb{R}^{n}$ e devolve $\mu=\Psi^{T} \lambda$ (linhas 5-13). Quando work $\neq T$, o Algoritmo 1 recebe como parâmetro de entrada $\lambda \in \mathbb{R}^{n}$ e devolve $\mu=\Psi \lambda$ (linhas 15-23).

Para calcular $\mu=\Psi^{T} \lambda=H_{1}^{T}\left(H_{2}^{T}\left(H_{3}^{T} \lambda\right)\right)$, o Algoritmo 1 pré-multiplica o vetor $\lambda$ pela matriz $H_{3}^{T}$, depois multiplica o resultado pela matriz $H_{2}^{T}$ e, finalmente, multiplica o resultado pela matriz $H_{1}^{T}$. Estas operações são realizadas no Algoritmo 1 no laço das linhas 5-13 para $i=1$, $i=2$ e $i=3$, respectivamente. Quer dizer, quando $i=1$ calcula-se $H_{3}^{T} \lambda$, quando $i=2$ aplica-se a transformação $H_{2}^{T}$ e quando $i=3$ aplica-se a transformação $H_{1}^{T}$. Basicamente, o produto $\mu=\Psi^{T} \lambda$ requer $\log _{2} n$ pré-multiplicações pelas matrizes $H_{i}^{T}$ e cada um desses produtos requer $2^{i}$ operações. A Tabela 3.1 mostra o custo computacional destas operações. Logo, o custo computacional de calcular $\mu=\Psi^{T} \lambda$ é dado por

$$
\begin{aligned}
T(n) & =O(1)+O(1)+O(n)+\left(\log _{2} n\right) O(1)+\left(\sum_{i=1}^{\log _{2} n} 2^{i-1}\right) O(1)+\left(\sum_{i=1}^{\log _{2} n} 2^{i}\right) O(1) \\
& =O(n)+O\left(\log _{2} n\right)+\left(\sum_{i=1}^{\log _{2} n} 2^{i-1}\right) O(1)+\left(\sum_{i=1}^{\log _{2} n} 2^{i}\right) O(1) \\
& =O(n)+O(n) O(1)+O(n) O(1) \\
& =O(n) .
\end{aligned}
$$

O cálculo do custo computacional para a avaliação de $\mu=\Psi \lambda$ (que aparecerá no cálculo do gradiente), que corresponde às linhas 15-23 é análogo e também é dado por $O(n)$.

Resta agora analisar, para completar a análise do custo da avaliação da função objetivo $F(z)$ de (3.3) definida em (3.6), o custo do produto $y=\Phi x$. Ilustraremos esta análise considerando a 


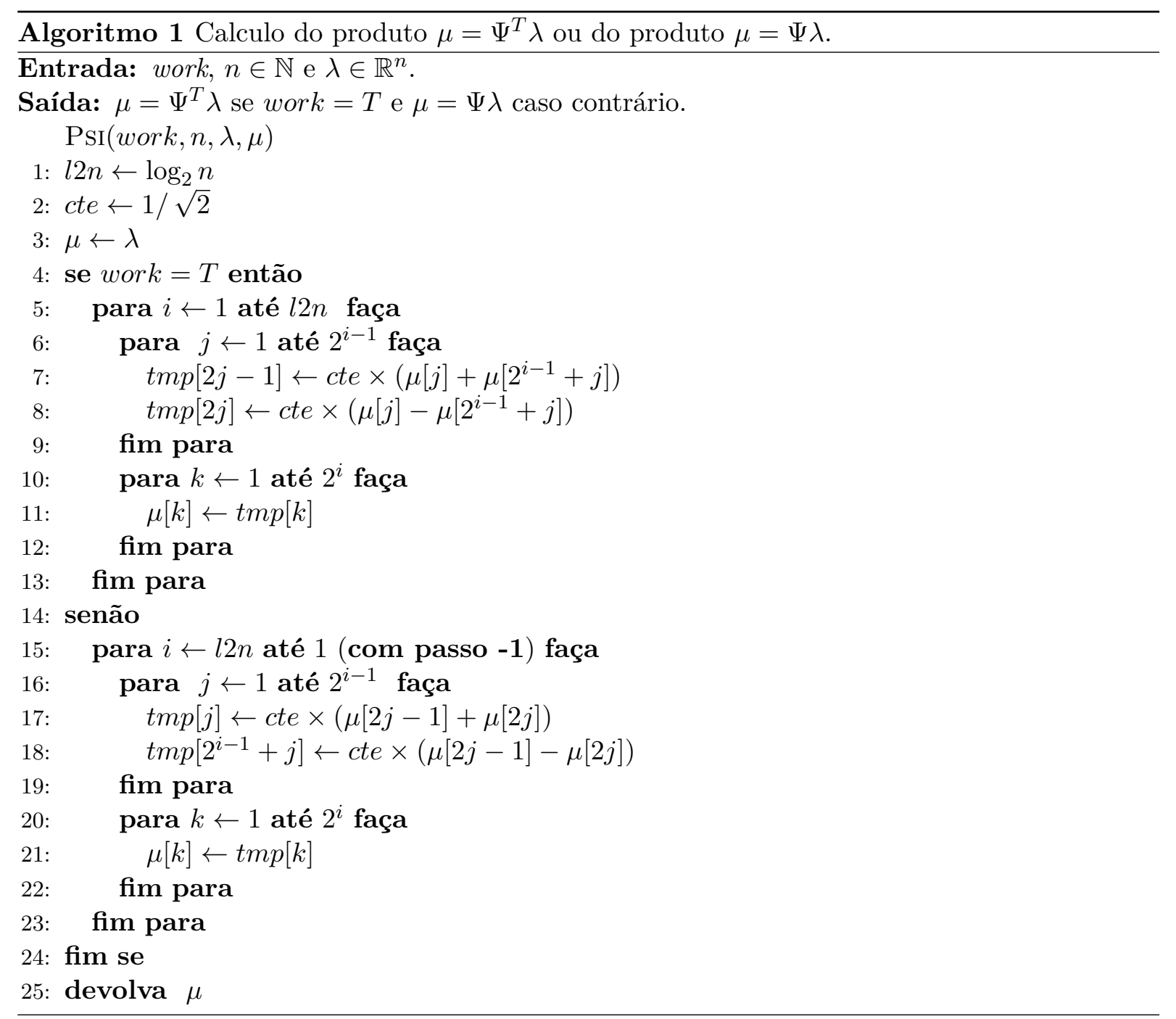

\begin{tabular}{ccc}
\hline linha & custo & \# vezes \\
\hline 1 & $O(1)$ & 1 \\
2 & $O(1)$ & 1 \\
3 & $O(n)$ & 1 \\
5 & $O(1)$ & $\log _{2} n$ \\
$6-9$ & $O(1)$ & $\sum_{i=1}^{\log _{2} n} 2^{i-1}$ \\
& & $\sum_{i=1}^{\log _{2} n} 2^{i}$ \\
\hline
\end{tabular}

Tabela 3.1: Custo computacional das linhas 5-13 do Algoritmo 1 que calculam o produto $\mu=\Psi^{T} \lambda$.

matriz de medição estudada no Capítulo 2 e dada por

$$
\Phi=\left[\begin{array}{c}
\mathbf{A}_{1} \\
\mathbf{A}_{2} \\
\vdots \\
\mathbf{A}_{L}
\end{array}\right]=\left[\begin{array}{c}
\hat{\mathbf{A}}_{1} \mathbf{P}_{1} \\
\hat{\mathbf{A}}_{2} \mathbf{P}_{2} \\
\vdots \\
\hat{\mathbf{A}}_{L} \mathbf{P}_{L}
\end{array}\right]=\left[\begin{array}{c}
\operatorname{diag}\left(\mathbf{a}_{1}^{1}, \ldots, \mathbf{a}_{m_{1}}^{1}\right) \times \mathbf{P}_{1} \\
\operatorname{diag}\left(\mathbf{a}_{1}^{2}, \ldots, \mathbf{a}_{m_{2}}^{2}\right) \times \mathbf{P}_{2} \\
\vdots \\
\operatorname{diag}\left(\mathbf{a}_{1}^{L}, \ldots, \mathbf{a}_{m_{L}}^{L}\right) \times \mathbf{P}_{L}
\end{array}\right],
$$


onde $L \leq m$ é um parâmetro dado, $\hat{\mathbf{A}}_{1}, \hat{\mathbf{A}}_{2}, \ldots, \hat{\mathbf{A}}_{L}$ são matrizes bloco diagonais de $m_{i} \times n$ com $\sum_{i=1}^{L} m_{i}=m$ e $m_{i}=\lfloor m / L\rfloor$ ou $m_{i}=\lfloor m / L\rfloor+1$ e $\mathbf{P}_{1}, \mathbf{P}_{2}, \ldots, \mathbf{P}_{L}$ são matrizes de permutação aleatórias de $n \times n$. Consideremos o seguinte exemplo de matriz de medição com $n=8, m=4$ e $L=2$ dada por

$$
\Phi=\left[\begin{array}{l}
\mathbf{A}_{1} \\
\mathbf{A}_{2}
\end{array}\right]=\left[\begin{array}{c}
\hat{\mathbf{A}}_{1} \mathbf{P}_{1} \\
\hat{\mathbf{A}}_{2} \mathbf{P}_{2}
\end{array}\right]
$$

onde

$$
\begin{aligned}
& \hat{\mathbf{A}}_{1}=\left(\begin{array}{llllllll}
1 & 1 & 1 & 0 & 0 & 0 & 0 & 0 \\
0 & 0 & 0 & 1 & 1 & 1 & 0 & 0 \\
0 & 0 & 0 & 0 & 0 & 0 & 1 & 1
\end{array}\right), \quad \hat{\mathbf{A}}_{2}=\left(\begin{array}{llllllll}
1 & 1 & 1 & 0 & 0 & 0 & 0 & 0 \\
0 & 0 & 0 & 1 & 1 & 1 & 0 & 0 \\
0 & 0 & 0 & 0 & 0 & 0 & 1 & 1
\end{array}\right), \\
& \mathbf{P}_{1}=\left(\begin{array}{llllllll}
0 & 0 & 0 & 0 & 0 & 0 & 0 & 1 \\
0 & 0 & 0 & 1 & 0 & 0 & 0 & 0 \\
0 & 0 & 0 & 0 & 0 & 0 & 1 & 0 \\
0 & 1 & 0 & 0 & 0 & 0 & 0 & 0 \\
0 & 0 & 1 & 0 & 0 & 0 & 0 & 0 \\
0 & 0 & 0 & 0 & 1 & 0 & 0 & 0 \\
1 & 0 & 0 & 0 & 0 & 0 & 0 & 0 \\
0 & 0 & 0 & 0 & 0 & 1 & 0 & 0
\end{array}\right), \quad \mathbf{P}_{2}=\left(\begin{array}{llllllll}
1 & 0 & 0 & 0 & 0 & 0 & 0 & 0 \\
0 & 0 & 0 & 0 & 0 & 0 & 0 & 1 \\
0 & 1 & 0 & 0 & 0 & 0 & 0 & 0 \\
0 & 0 & 0 & 0 & 0 & 0 & 1 & 0 \\
0 & 0 & 0 & 0 & 1 & 0 & 0 & 0 \\
0 & 0 & 1 & 0 & 0 & 0 & 0 & 0 \\
0 & 0 & 0 & 1 & 0 & 0 & 0 & 0 \\
0 & 0 & 0 & 0 & 0 & 1 & 0 & 0
\end{array}\right),
\end{aligned}
$$

quer dizer

$$
\Phi=\left(\begin{array}{llllllll}
0 & 0 & 0 & 1 & 0 & 0 & 1 & 1 \\
0 & 1 & 1 & 0 & 1 & 0 & 0 & 0 \\
1 & 0 & 0 & 0 & 0 & 1 & 0 & 0 \\
1 & 1 & 0 & 0 & 0 & 0 & 0 & 1 \\
0 & 0 & 1 & 0 & 1 & 0 & 1 & 0 \\
0 & 0 & 0 & 1 & 0 & 1 & 0 & 0
\end{array}\right)
$$

Cada matriz de permutação $P_{i}, i=1, \ldots, L$, pode ser armazenada como uma coluna de uma matriz $\mathbf{P} \in \mathbb{R}^{n \times L}$. As matrizes $\mathbf{P}_{1}$ e $\mathbf{P}_{2}$ podem ser representadas por vetores de permutação da seguinte maneira

$$
p_{1}=\left(\begin{array}{llllllll}
7 & 4 & 5 & 4 & 6 & 8 & 8 & 8
\end{array}\right)^{T} \text { e } p_{2}=\left(\begin{array}{llllllll}
1 & 3 & 6 & 7 & 5 & 8 & 7 & 8
\end{array}\right)^{T} .
$$

Logo, a matriz $\mathbf{P}$ é dada por

$$
\mathbf{P}=\left[\begin{array}{l}
p_{1}^{T} \\
p_{2}^{T}
\end{array}\right]^{T}=\left(\begin{array}{llllllll}
7 & 4 & 5 & 4 & 6 & 8 & 8 & 8 \\
1 & 3 & 6 & 7 & 5 & 8 & 7 & 8
\end{array}\right)^{T}
$$

Dado $\omega \in \mathbb{R}^{n}$, calcular $P_{j} \omega$ usando a $j$-esima coluna da matriz $\mathbf{P}$ consiste em fazer: para $i=1, \ldots, n-1$, permutar $\omega_{i}$ com $\omega_{P_{i j}}$. Claramente, esta operação tem custo $O(n)$. O produto $P_{j}^{T} \omega$ consistem em fazer: para $i=n-1, \ldots, 1$ (com passo -1 ), permutar $\omega_{i}$ com $\omega_{P_{i j}}$. O 
Algoritmo 2 implementa estas operações.

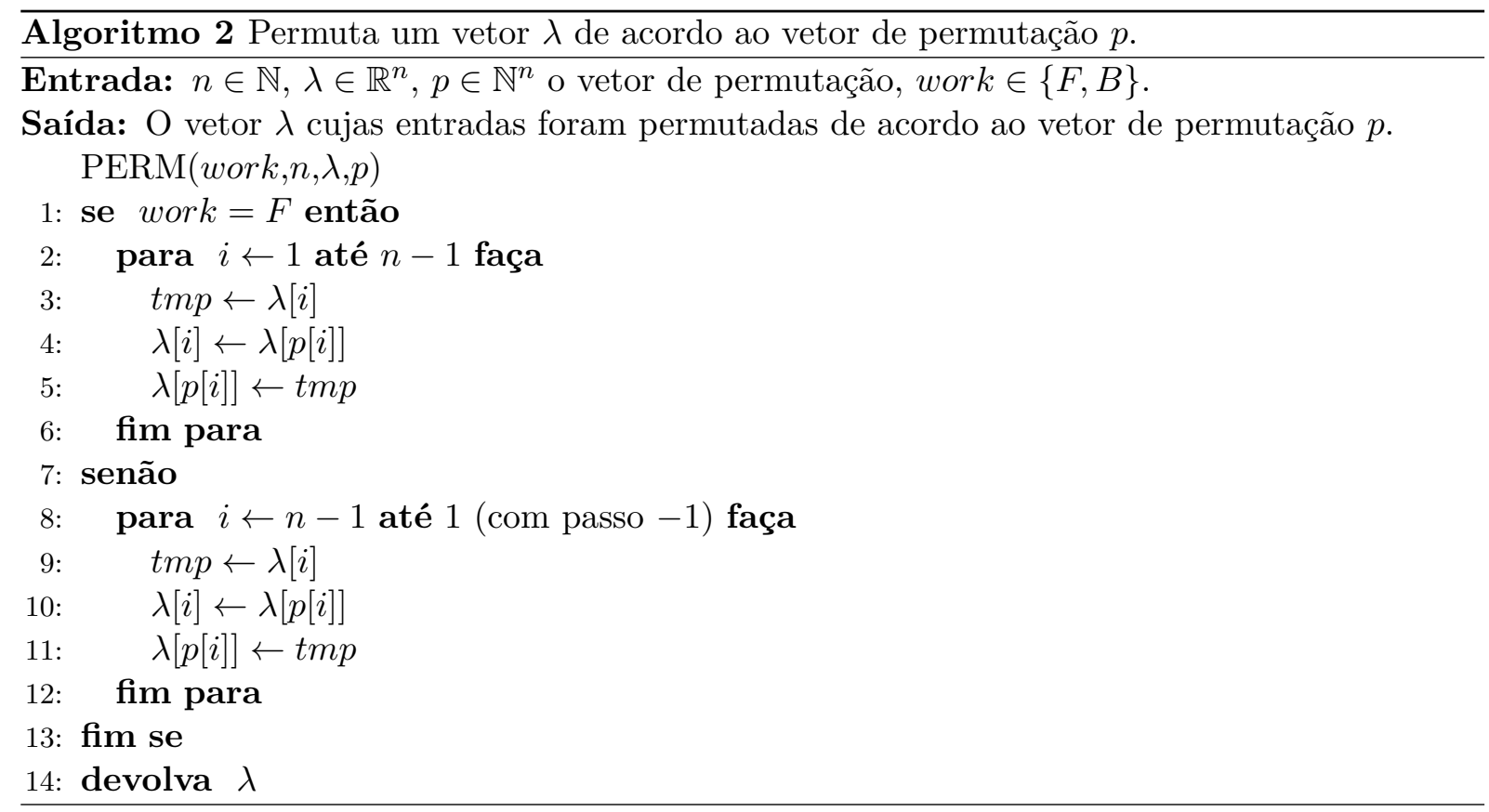

Para calcular $y=\Phi x$ no nosso exemplo, realizamos as seguintes operações

$$
y=\Phi x=\left[\begin{array}{c}
\hat{\mathbf{A}}_{1} \mathbf{P}_{1} \\
\hat{\mathbf{A}}_{2} \mathbf{P}_{2}
\end{array}\right] x=\left[\begin{array}{c}
\hat{\mathbf{A}}_{1}\left(\mathbf{P}_{1} x\right) \\
\hat{\mathbf{A}}_{2}\left(\mathbf{P}_{2} x\right)
\end{array}\right] .
$$

Quer dizer, pré-multiplicamos o vetor $x$ pelas matrizes de permutação $\mathbf{P}_{1}$ e $\mathbf{P}_{2}$, pré-multiplicamos depois pelas matrizes bloco-diagonais $\hat{\mathbf{A}}_{1}$ e $\hat{\mathbf{A}}_{2}$ e finalmente "concatenamos" os resultados.

Como já mencionamos acima, o custo de pré-multiplicar por cada matriz de permutação é $O(n)$. A regra de formação das matrizes $\mathbf{A}_{i}$ permite que cada produto seja calculado em $O(n L)$ sem sequer precisar armazenar a matriz $\mathbf{A}_{i}$. O Algoritmo 3 implementa o produto $y=\Phi x$ e o produto $\hat{x}=\Phi^{T} y$ (que será necessário no calculo do gradiente da função objetivo), dependendo do valor do parâmetro work. Quando work $=T$, o Algoritmo 3 recebe como parâmetro de entrada $\lambda \in \mathbb{R}^{m}$ e devolve $\mu=\Phi^{T} \lambda$. Quando work $\neq T$, o Algoritmo 3 recebe como parâmetro de entrada $\lambda \in \mathbb{R}^{n}$ e devolve $\mu=\Phi \lambda$. A Tabela 3.2 mostra o custo computacional destas operações. Logo, o custo computacional de calcular $\mu=\Phi \lambda$ é dado por

$$
\begin{aligned}
T(n)= & O(1)+O(1)+O(1)+L O(1)+L O(n)+L O(n)+L O(1)+n O(1)+ \\
& n O(1)+n O(1)+n L O(1)+L O(1) \\
= & O(1)+O(L)+O(n L)+O(L)+O(n)+O(n)+O(n)+O(n L)+O(L) \\
= & O(L)+O(n)+O(n L) \\
= & O(n L) .
\end{aligned}
$$

A analise do cálculo de $\mu=\Phi^{T} \lambda$ é análoga à análise do cálculo de $\mu=\Phi \lambda$ e é fácil ver que o 


\begin{tabular}{ccc}
\hline linha & custo & \# vezes \\
\hline 1 & $O(1)$ & 1 \\
2 & $O(1)$ & 1 \\
3 & $O(1)$ & 1 \\
$4-9$ & $O(1)$ & $L$ \\
$10-12$ & $O(n)$ & $L$ \\
$13-15$ & $O(n)$ & $L$ \\
$16-18$ & $O(1)$ & $L$ \\
19 & $O(1)$ & $n$ \\
$20-24$ & $O(1)$ & $n$ \\
30 & $O(1)$ & $n$ \\
$31-33$ & $O(1)$ & $n L$ \\
39 & $O(1)$ & $L$
\end{tabular}

Tabela 3.2: Custo computacional do Algoritmo 3 no cálculo do produto $\mu=\Phi \lambda$.

seu custo é também $O(n L)$.

Havendo analisado os custos computacionais de pré-multiplicar pelas matrizes $\Phi$ e $\Psi$ (ou suas transpostas), podemos concluir que o custo de pré-multiplicar por $\Theta$ ou $\Theta^{T}$ é $O(n L)$. Desta forma, o custo de avaliação da função objetivo do problema (3.3) é $O(n L)$.

Concluímos esta seção analisando o custo de avaliar o gradiente da função objetivo do problema (3.3) dado por

$$
\nabla F(z)=(1-\gamma) B z+c
$$

com $B$ e $c$ como definidos em (3.4). Denotando $z=(u, v)^{T}$, temos que

$$
\nabla F(z)=(1-\gamma)\left[\begin{array}{r}
\Theta^{T} \Theta(u-v) \\
-\Theta^{T} \Theta(u-v)
\end{array}\right]+c
$$

É fácil ver que o cálculo do gradiente da função objetivo (3.3) requer uma pré-multiplicação pela matriz $\Theta$ e uma pré-multiplicação pela matriz $\Theta^{T}$. Logo, o custo de calcular o gradiente é também $O(n L)$. Mais ainda, se armazenássemos o produto pela matriz $\Theta$ utilizado na avaliação da função objetivo, apenas um único produto adicional seria necessário.

\subsection{Gradiente Espectral Projetado}

O método do Gradiente Espectral Projetado (SPG) aplica-se ao problema

$$
\text { Minimizar } f(x) \text { sujeita a } x \in \Omega \text {, }
$$

onde $f: \mathbb{R}^{n} \rightarrow \mathbb{R}$ é continua e diferenciável e $\Omega$ é um conjunto convexo. Por utilizar apenas informação de primeira ordem e precisar de uns poucos vetores de tamanho $n$ na sua implementação, o SPG é adequado para problemas de grande porte. A eficiência do método esta diretamente relacionada à existência de uma forma eficiente de projetar um ponto arbitrário $x \in \mathbb{R}^{n}$ no 


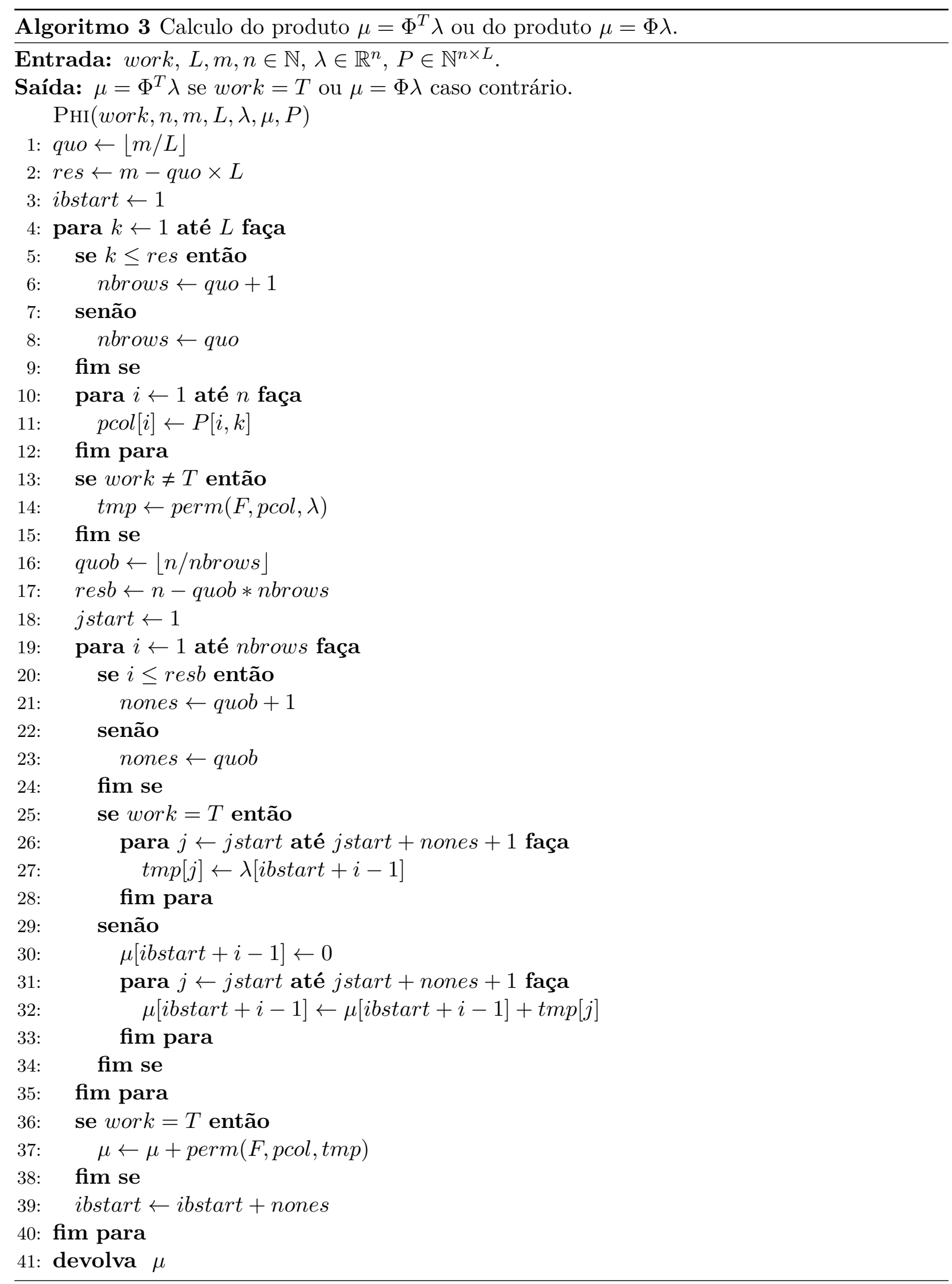

conjunto convexo $\Omega$. O método do Gradientes Espectral Projetado foi introduzido em [12, 16] e combina o método clássico de gradiente projetado com dois ingredientes: o passo de Barzilai- 
Borwein [6] e a busca linear não-monótona de Grippo, Lampariello e Lucidi [39]. Na ultima década, o SPG foi utilizado com sucesso em diversas aplicações práticas. Dentre elas destacamos a resolução dos problemas de otimização associados a máquinas de suporte vetorial [25, 49], ao calculo de perturbações condicionais não-lineares ótimas [46] e a compressive sensing [31, 33], dentre outros. A seguir, descrevemos brevemente o método SPG.

As iterações do método SPG são da forma

$$
x_{k+1}=x_{k}+\alpha_{k} d_{k},
$$

onde

$$
d_{k}=P_{\Omega}\left(x_{k}-\lambda_{k} \nabla f\left(x_{k}\right)\right)-x_{k}
$$

e $\alpha_{k}$ é tal que $x_{k+1}$ satisfaz a condição de Armijo não monótona dada por

$$
f\left(x_{k+1}\right) \leq f_{\max }+\alpha_{k} \gamma_{\operatorname{arm}} \nabla f\left(x_{k}\right)^{T} d_{k} .
$$

$\operatorname{Em}(3.9), P_{\Omega}(\cdot)$ representa a projeção Euclidiana no conjunto convexo $\Omega$, i.e.

$$
P_{\Omega}(\tilde{x})=\arg \min _{x} \frac{1}{2}\|x-\tilde{x}\|_{2}^{2} \text { sujeita a } x \in \Omega,
$$

e $\lambda_{k}$ é o passo espectral dado por

$$
\lambda_{k}=\max \left\{\lambda_{\min }, \min \left\{s_{k-1}^{T} s_{k-1} / s_{k-1}^{T} y_{k-1}, \lambda_{\max }\right\}\right\}
$$

onde $s_{k-1}=x_{k}-x_{k-1}, y_{k-1}=\nabla f\left(x_{k}\right)-\nabla f\left(x_{k-1}\right)$ e $0<\lambda_{\min } \leq \lambda_{\max }<+\infty$ são salvaguardas dadas. Na pratica, utiliza-se $\lambda_{\min }=10^{-30}$ e $\lambda_{\max }=10^{30}$.

Em (3.10) $\gamma_{\text {arm }}>0$ é a constante de Armijo (tipicamente $\gamma_{\text {arm }}=10^{-4}$ ),

$$
f_{\max }=\max \left\{f\left(x_{k-j}\right) \mid 0 \leq j \leq \min \{k, M-1\}\right\}
$$

e $M \geq 1$ é uma constante que regula a não monotonicidade da busca linear. A condição (3.10) garante decréscimo suficiente a cada $M$ iterações. Se $M=1$ então (3.10) coincide com o critério clássico de Armijo. Em [12] sugere-se $M=10$ como valor default.

O paso espectral $\lambda_{k}$ definido em (3.11) relaciona o método SPG com os métodos do tipo secante. Os métodos quase-Newton [32] do tipo secante obedecem a fórmula $x_{k+1}=x_{k}+\alpha_{k} d_{k}$ com

$$
d_{k}=B_{k}^{-1} \nabla f\left(x_{k}\right),
$$

onde as matrizes $B_{k}$ satisfazem a equação secante

$$
B_{k} s_{k-1}=y_{k-1} .
$$


Naturalmente, o custo de resolver o sistema linear (3.12) é o fator decisivo do custo da iteração dos métodos quase-Newton e a escolha da matriz $B_{k}$ pode fazer o método estar tão próximo de um método do tipo gradiente o do método de Newton quanto desejado. Se impusermos $B_{k}=\tau I$ então a equação secante (3.13) fica $\tau s_{k-1}=y_{k-1}$. Esta equação muito provavelmente não terá solução, mas se considerarmos a solução de quadrados mínimos teremos

$$
\tau_{k}=s_{k-1}^{T} y_{k-1} / s_{k-1}^{T} s_{k-1}
$$

quer dizer, $B_{k}=\tau_{k} I$ e $d_{k}=\hat{\lambda}_{k} \nabla f\left(x_{k}\right)$ com $\hat{\lambda}_{k}=1 / \tau_{k}=s_{k-1}^{T} s_{k-1} / s_{k-1}^{T} y_{k-1}$. O passo espectral $\lambda_{k}$ em (3.11) é a projeção de $\hat{\lambda}_{k}$ no intervalo $\left[\lambda_{\min }, \lambda_{\max }\right]$. Quer dizer, o passo espectral proporciona ao SPG algum tipo de informação de segunda ordem com custo mínimo. O uso da busca linear não monótona do tipo Armijo (no lugar da versão monótona) faz com que a primeira tentativa de passo na direção $d_{k}$, que como acabou de ser explicado carrega alguma informação de segunda ordem, tenha mais chances de ser aceita.

Sejam $M \geq 1,0<\lambda_{\min } \leq \lambda_{\max }<+\infty, 0<\sigma_{1} \leq \sigma_{2}<1, \gamma_{\text {arm }}>0$ e $\varepsilon>0$ parâmetros dados. $\mathrm{O}$ método começa com $x_{0} \in \Omega$ e $\lambda_{0} \in\left[\lambda_{\min }, \lambda_{\max }\right]$ arbitrários. Dados $x_{k} \in \Omega$ e $\lambda_{k} \in\left[\lambda_{\min }, \lambda_{\max }\right]$, o Algoritmo 4 descreve como obter $x_{k+1}$ e $\lambda_{k+1}$ e como terminar o processo.

\section{Algoritmo 4.}

Passo 1. Critério de parada

Se $\left\|P_{\Omega}\left(x_{k}-\nabla f\left(x_{k}\right)\right)-x_{k}\right\| \leq \varepsilon$, pare declarando que $x_{k}$ é um ponto estacionário.

Passo 2. Backtracking

Passo 2.1. Calcule $d_{k}=P_{\Omega}\left(x_{k}-\lambda_{k} \nabla f\left(x_{k}\right)\right)-x_{k}$ e faça $\alpha \leftarrow 1$.

Passo 2.2. Se

$$
f\left(x_{k}+\alpha d_{k}\right) \leq \max _{0 \leq j \leq \min \{k, M-1\}} f\left(x_{k-j}\right)+\gamma_{\operatorname{arm}} \alpha \nabla f\left(x_{k}\right)^{T} d_{k}
$$

defina $\alpha_{k}=\alpha, x_{k+1}=x_{k}+\alpha d_{k}, s_{k}=x_{k+1}-x_{k}, y_{k}=\nabla f\left(x_{k+1}\right)-\nabla f\left(x_{k}\right)$ e va para o Passo 3 .

Caso contrário, defina $\alpha_{\text {new }} \in\left[\sigma_{1} \alpha, \sigma_{2} \alpha\right]$, faça $\alpha \leftarrow \alpha_{\text {new }}$ e volte ao inicio do Passo 2.2.

Passo 3. Cálculo do passo espectral

Se $s_{k}^{T} y_{k} \leq 0$, defina $\lambda_{k+1}=\lambda_{\max }$, senão, defina $\lambda_{k+1}=\max \left\{\lambda_{\min }, \min \left\{s_{k}^{T} s_{k} / s_{k}^{T} y_{k}, \lambda_{\max }\right\}\right\}$.

No Passo 2.2, o cálculo de $\alpha_{\text {new }}$ utiliza uma interpolação quadrática unidimensional salvaguardada tomando passo $\alpha_{\text {new }}=\alpha / 2$ quando o minimizador da quadrática unidimensional fica fora do intervalo $\left[\sigma_{1}, \sigma_{2} \alpha\right]$. Note que o processo de salvaguarda baseia-se em $\left[\sigma_{1}, \sigma_{2} \alpha\right]$ no lugar da escolha usual $\left[\sigma_{1} \alpha, \sigma_{2} \alpha\right]$. Isto significa que, quando a interpolação tende a rejeitar $90 \%$ (para $\left.\sigma_{1}=0.1\right)$ do intervalo original $[0,1]$, julga-se que a previsão não é confiável a o processo mais conservativo de biseção é utilizado. Experimentos numéricos em [12] mostraram que este procedimento obtêm (ao menos para os problemas considerados em [12]) melhores resultados do que 
o procedimento clássico. O Algoritmo 5 descreve a busca linear em detalhe.

\section{Algoritmo 5.}

Passo 1. Calcule $f_{\max }=\max \left\{f\left(x_{k-j}\right) \mid 0 \leq j \leq \min \{k, M-1\}\right\}$ e $\delta=\nabla f\left(x_{k}\right)^{T} d_{k}$ e faça $\alpha \leftarrow 1$.

Passo 2. Faça $x_{+} \leftarrow x_{k}+\alpha d_{k}$. Se

$$
f\left(x_{+}\right) \leq f_{\max }+\gamma_{\operatorname{arm}} \alpha \delta
$$

faça $\alpha_{k}=\alpha$ e termine.

Passo 3. Faça $\alpha_{\mathrm{tmp}}=\frac{1}{2} \alpha^{2} \delta /\left(f\left(x_{+}\right)-f\left(x_{k}\right)-\alpha \delta\right)$.

Se $\alpha_{\text {tmp }} \geq \sigma_{1}$ e $\alpha_{\text {tmp }} \leq \sigma_{2} \alpha$ então faça $\alpha \leftarrow \alpha_{\text {tmp }}$. Caso contrário, faça $\alpha \leftarrow \alpha / 2$. Va para o Passo 2.

A boa definição e a teoria de convergência do SPG pode ser encontrada em [12], onde também são descritos experimentos numéricos relacionados à aplicação do SPG a problemas de minimização em caixas. Em [16] experimentos numéricos com uma família de problemas de grande porte (com milhões de variáveis e restrições) associados à alocação de pontos no plano são apresentados. A extensão do SPG para o caso de projeções inexatas foi apresentada em [13] e a extensão para problemas com restrições lineares foi introduzida em [1]. Para uma contextualização histórica do SPG e uma analise detalhada das suas variantes, veja [14]. 


\section{Capítulo 4}

\section{Experimentos computacionais}

Neste capítulo apresentamos experimentos computacionais relacionados a recuperar um sinal a partir do sinal amostrado. Na Seção 4.1 analisamos com experimentos preliminares a influência do valor do parâmetro $L$ na composição da matriz de amostragem $\Phi$ definida no Capítulo 2 . Na Seção 4.2 abordamos os problemas de programação linear (2.19) provenientes de considerar um ambiente em que o sinal é amostrado sem ruido. O caso em que há ruido na amostragem do sinal, e o problema a ser resolvido é o problema (3.3), é considerado na Seção 4.3. Finalmente, a Seção 4.4 inclui experimentos com problemas de grande porte.

Para a resolução dos problemas de programação linear (2.19) consideraremos os software CPLEX (IBM ILOG AMPL/CPLEX 12.1.0) [9] e Algencan 2.4.0 [3, 4]. Para os problemas do tipo (3.3) consideraremos o método do Gradiente Espectral Projetado [12, 16]. Algencan e SPG estão programados na linguagem Fortran 77 e foram compilados com o GNU Fortran (gfortran) versão 4.6.1 utilizando a opção de compilação "-O4". Todos os experimentos deste capítulo foram conduzidos em um computador com processador InTEL XeON com 2.67GHz e 8GB de memória RAM, rodando o sistema operacional GNU/LinUX.

\subsection{Experimentos preliminares}

Nesta seção estamos interessados em avaliar a influência do parâmetro $L$ na construção da matriz de medição $\Phi$ do tipo BPBD (veja a equação (2.29) na Seção 2.3).

Consideremos a imagem $x^{*}$ com $n=4 \times 8=32$ pixels da Figura 4.1. O sinal $x^{*} \in \mathbb{R}^{32}$ tem componentes $x_{i}^{*} \in[0,1]$ que representam uma escala de cinza $(0=$ preto e $1=$ branco $)$. Se considerarmos como base a matriz $\Psi \in \mathbb{R}^{32 \times 32}$ associada às funções base da transformada de Haar, temos que $x^{*}$ pode ser representado como $x^{*}=\Psi^{T} s^{*}$ com

$$
s^{*}=(4.24,0,1,-1,0,0,0,0,0,0,1,-1,1,-1,0,0,0,0,0,0,0,0,0,0,0,0,0,0,0)^{T} .
$$

Note que, conhecendo $x^{*}$ e $\Psi$, o valor de $s^{*}$ pode ser calculado simplesmente como $s^{*}=\Psi x^{*}$. Para $s^{*}$ temos que $k=\left\|s^{*}\right\|_{0}=7$ e, se definimos a densidade de $x^{*}$ como

$$
\delta\left(x^{*}\right)=\frac{\left\|s^{*}\right\|_{0}}{n} \times 100 \%,
$$


temos que a $\delta\left(x^{*}\right)=7 / 32 \times 100 \%=21.875 \%$.

\begin{tabular}{|c|c|c|c|c|c|}
\hline \begin{tabular}{l|l}
1.0 & 1.
\end{tabular} & 1.01 .0 & 1.0 & 1.0 & 1.0 & 1.0 \\
\hline & $\begin{array}{ll}1.0 & 0.0\end{array}$ & 0.0 & 0.0 & & \\
\hline $\begin{array}{ll}1.0 & 1 .\end{array}$ & $\begin{array}{l}1.0 \\
0.0\end{array}$ & 0.0 & & 0.0 & \\
\hline $\begin{array}{ll}1.0 & 1 .\end{array}$ & & & & & 1.0 \\
\hline
\end{tabular}

Figura 4.1: Sinal $x^{*}$ com $n=4 \times 8=32$ pixels.

O problema (2.19) pode ser reformulado como um problema de programação linear substituindo $s$ por $s=u-v$ com $u \geq 0$ e $v \geq 0$ como segue:

$$
\begin{array}{ll}
\text { Minimizar } & \sum_{i=1}^{n} u_{i}+\sum_{i=1}^{n} v_{i} \\
\text { sujeita a } & \Theta(u-v)=y \\
& u, v \geq 0
\end{array}
$$

onde $\Theta=\Phi \Psi^{T} \in \mathbb{R}^{m \times n}$, $\Phi$ é a matriz de medição do tipo BPBD com parâmetro $L$ e $\Psi$ é a matriz associada às funções base da transformada de Haar.

Desejamos agora, resolver diferentes problemas de programação linear da forma (4.3) para recuperar a imagem $x^{*}$ a partir de experimentos simulados com diferentes valores $m$ para o número de amostras e valores do parâmetro $L$ na construção da matriz de amostragem $\Phi$ do tipo BPBD (2.29). Consideramos então $m \in\{k, 1.5 k, 2 k, 2.5 k, 3 k\}$ e $L \in\{1,2,3\}$ e, usando as 15 combinações de $m$ e $L$, geramos as matrizes de amostragem $\Phi$ obtendo 15 problemas de programação linear diferentes. Os problemas foram resolvidos utilizando CPLEX com todos os seus parâmetros default que, em todos os casos, demorou menos do que uma centésima de segundo de tempo de CPU. As Tabelas 4.1-4.3 mostram os coeficientes $s=u-v$ encontrados e as Figuras 4.2-4.4 ilustram as imagens reconstruídas utilizando os coeficientes encontrados.

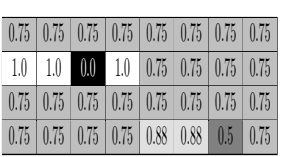

(a) $m=k$

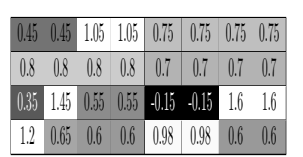

(b) $m=1.5 k$

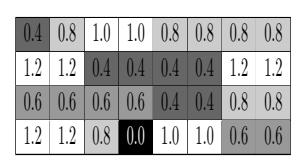

(c) $m=2 k$

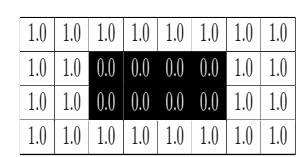

(d) $m=2.5 k$

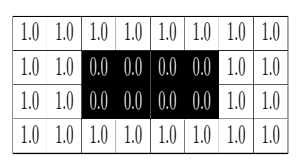

(e) $m=3 k$

Figura 4.2: Imagens reconstruidas utilizando os coeficientes recuperados nos problemas de programação linear com matriz de medição $\Phi$ do tipo BPBD com $L=1$. Os coeficientes recuperados correspondem aos mostrados na Tabela 4.1.

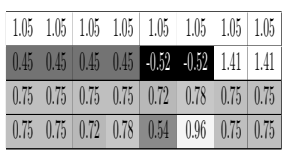

(a) $m=k$

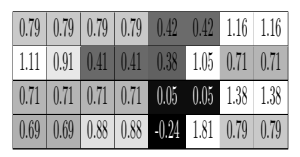

(b) $m=1.5 k$

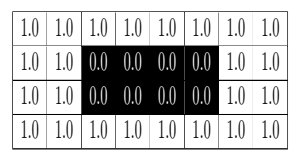

(c) $m=2 k$

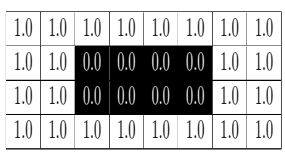

(d) $m=2.5 k$

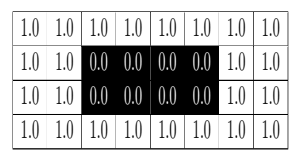

(e) $m=3 k$

Figura 4.3: Imagens reconstruidas utilizando os coeficientes recuperados nos problemas de programação linear com matriz de medição $\Phi$ do tipo BPBD com $L=2$. Os coeficientes recuperados correspondem aos mostrados na Tabela 4.2.

É importante notar que, dado $s=u-v$ solução (coeficientes recuperados) de um problema de 


\begin{tabular}{|c|c|c|c|c|c|c|}
\hline & \multirow{2}{*}{$\mathrm{i}$} & \multicolumn{5}{|c|}{ Número de amostras } \\
\hline & & $m=k$ & $m=1.5 k$ & $m=2 k$ & $m=2.5$ & $m=3 k$ \\
\hline \multirow{32}{*}{${ }^{*} \infty$} & 1 & 4.2426406871192910 & 4.2426406868698239 & 4.2426406871192892 & 4.2426406865388406 & 4.2426406871116527 \\
\hline & 2 & 0.0000000000000000 & 0.0000000000000000 & 0.2828427124747471 & 0.0000000000000000 & 0.0000000000000000 \\
\hline & 3 & 0.0000000000000000 & 0.0000000000000000 & 0.0000000000000000 & 0.9999999810654676 & 0.9999999999853667 \\
\hline & 4 & 0.0000000000000000 & -0.1000000045880174 & -0.3999999999999330 & -0.9999999895130788 & -1.0000000000307061 \\
\hline & 5 & 0.0000000000000000 & 0.0000000000000000 & 0.0000000000000000 & 0.0000000000000000 & 0.0000000000000000 \\
\hline & 6 & 0.0000000000000000 & 0.1414213581233788 & 0.0000000000000000 & 0.0000000000000000 & 0.0000000000000000 \\
\hline & 7 & 0.0000000000000000 & 0.0000000000000000 & 0.0000000000000000 & 0.0000000000000000 & 0.0000000000000000 \\
\hline & 8 & 0.0000000000000000 & $-3.53182290708 \mathrm{E}-002$ & 0.0000000000000000 & 0.0000000000000000 & 0.0000000000000000 \\
\hline & 9 & 0.0000000000000000 & -0.5999999999159670 & -0.4000000000001712 & 0.0000000000000000 & 0.0000000000000000 \\
\hline & 10 & 0.0000000000000000 & 0.0000000000000000 & 0.0000000000000000 & 0.0000000000000000 & 0.0000000000000000 \\
\hline & 11 & 0.4999999999998990 & 0.0000000000000000 & 0.7999999999998720 & 0.9999999877722606 & 0.9999999999990342 \\
\hline & 12 & 0.0000000000000000 & 0.0000000000000000 & -0.7999999999999475 & -0.9999999963054850 & -0.9999999999822905 \\
\hline & 13 & 0.0000000000000000 & 0.3500000008558777 & 0.0000000000000000 & 0.9999999850468577 & 1.0000000000056701 \\
\hline & 14 & 0.0000000000000000 & -1.7499999944837192 & -0.4000000000000801 & -0.9999999909906753 & -1.0000000000374079 \\
\hline & 15 & 0.0000000000000000 & 0.3250000005176638 & 0.7999999999998675 & $7.88522282224 \mathrm{E}-009$ & 0.0000000000000000 \\
\hline & 16 & 0.2500000000000370 & 0.3749999983975413 & 0.3999999999999633 & 0.0000000000000000 & 0.0000000000000000 \\
\hline & 17 & 0.0000000000000000 & 0.0000000000000000 & -0.2828427124745003 & $9.91059873883 \mathrm{E}-009$ & 0.0000000000000000 \\
\hline & 18 & 0.0000000000000000 & 0.0000000000000000 & 0.0000000000000000 & 0.0000000000000000 & 0.0000000000000000 \\
\hline & 19 & 0.0000000000000000 & 0.0000000000000000 & 0.0000000000000000 & 0.0000000000000000 & 0.0000000000000000 \\
\hline & 20 & 0.0000000000000000 & 0.0000000000000000 & 0.0000000000000000 & 0.0000000000000000 & 0.0000000000000000 \\
\hline & 21 & 0.0000000000000000 & 0.0000000000000000 & 0.0000000000000000 & 0.0000000000000000 & 0.0000000000000000 \\
\hline & 22 & -0.7071067811864443 & 0.0000000000000000 & 0.0000000000000000 & 0.0000000000000000 & 0.0000000000000000 \\
\hline & 23 & 0.0000000000000000 & 0.0000000000000000 & 0.0000000000000000 & 0.0000000000000000 & 0.0000000000000000 \\
\hline & 24 & 0.0000000000000000 & 0.0000000000000000 & 0.0000000000000000 & 0.0000000000000000 & 0.0000000000000000 \\
\hline & 25 & 0.0000000000000000 & -0.7778174603837905 & 0.0000000000000000 & 0.0000000000000000 & 0.0000000000000000 \\
\hline & 26 & 0.0000000000000000 & 0.0000000000000000 & 0.0000000000000000 & 0.0000000000000000 & 0.0000000000000000 \\
\hline & 27 & 0.0000000000000000 & 0.0000000000000000 & 0.0000000000000000 & 0.0000000000000000 & 0.0000000000000000 \\
\hline & 28 & 0.0000000000000000 & 0.0000000000000000 & 0.0000000000000000 & 0.0000000000000000 & 0.0000000000000000 \\
\hline & 29 & 0.0000000000000000 & 0.3889087267051568 & 0.0000000000000000 & 0.0000000000000000 & 0.0000000000000000 \\
\hline & 30 & 0.0000000000000000 & 0.0000000000000000 & 0.5656854249492100 & 0.0000000000000000 & 0.0000000000000000 \\
\hline & 31 & 0.0000000000000000 & 0.0000000000000000 & 0.0000000000000000 & 0.0000000000000000 & 0.0000000000000000 \\
\hline & 32 & -0.1767766952967148 & 0.0000000000000000 & 0.0000000000000000 & 0.0000000000000000 & 0.0000000000000000 \\
\hline
\end{tabular}

Tabela 4.1: Coeficientes recuperados considerando $m \in\{k, 1.5 k, 2 k, 2.5 k, 3 k\}$ amostras e uma matriz de medição $\Phi$ do tipo BPBD com $L=1$.

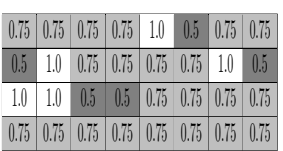

(a) $m=k$

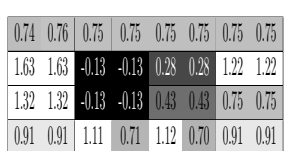

(b) $m=1.5 k$

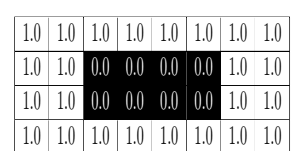

(c) $m=2 k$

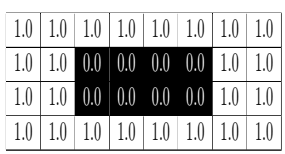

(d) $m=2.5 k$

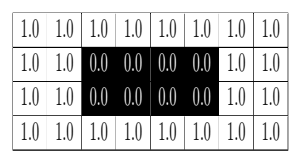

(e) $m=3 k$

Figura 4.4: Imagens reconstruídas utilizando os coeficientes recuperados nos problemas de programação linear com matriz de medição $\Phi$ do tipo BPBD com $L=3$. Os coeficientes recuperados correspondem aos mostrados na Tabela 4.3.

programação linear da forma (2.19) e dado $x=\Psi^{T} s$ (sinal construído a partir dos coeficientes recuperados ou simplesmente "sinal recuperado"), não há nada que implique que teremos $x_{i} \in$ $[0,1]$ como de fato acontece no sinal original. De fato, isso nem sempre ocorre, como pode ser observado na Figura 4.2b. Logo, as cores atribuídas aos pixels das Figuras 4.2-4.4 correspondem à projeção do valor calculado $x_{i}=\left[\Psi^{T} s\right]_{i}$ no intervalo $[0,1]$, quer dizer, na representação gráfica das imagens recuperadas mostramos $P_{[0,1]^{n}}(x)$, onde $P_{[0,1]^{n}}(\cdot)$ representa o operador de projeção na caixa $n$-dimensional $0 \leq x_{i} \leq 1, i=1, \ldots, n$.

A Figura 4.5 ilustra a diferença entre a imagem original e as imagens recuperadas ao resolver os 15 PLs para os diferentes valores de $m$ e $L$. Analisando as imagens recuperadas no caso $L=1$ (Figura 4.2) observamos que apenas nos casos $m \in\{2.5 k, 3 k\}$ o sinal original $x^{*}$ foi recuperado. 


\begin{tabular}{|c|c|c|c|c|c|c|}
\hline & \multirow{2}{*}{$\mathrm{i}$} & \multicolumn{5}{|c|}{ Número de amostras } \\
\hline & & $m=k$ & $m=1.5 k$ & $m=2 k$ & $m=2.5$ & $m=3 k$ \\
\hline \multirow{32}{*}{ * } & 1 & 4.2426406871172375 & 4.2426406871192874 & 4.2426406871210993 & 4.2426406870239237 & 4.2426406871132922 \\
\hline & 2 & 0.0000000000000000 & 0.0000000000000000 & 0.0000000000000000 & 0.0000000000000000 & 0.0000000000000000 \\
\hline & 3 & 1.2142857142597530 & 0.1578947368421057 & 1.0000000000149847 & 0.9999999999581101 & 1.0000000000169658 \\
\hline & 4 & 0.0000000000000000 & -0.1428571428571425 & -1.0000000000119675 & -0.9999999998935067 & -1.0000000000305638 \\
\hline & 5 & 0.0000000000000000 & 0.0000000000000000 & 0.0000000000000000 & 0.0000000000000000 & 0.0000000000000000 \\
\hline & 6 & 0.0000000000000000 & 0.0000000000000000 & 0.0000000000000000 & 0.0000000000000000 & 0.0000000000000000 \\
\hline & 7 & 0.0000000000000000 & 0.0000000000000000 & 0.0000000000000000 & 0.0000000000000000 & 0.0000000000000000 \\
\hline & 8 & 0.0000000000000000 & 0.0000000000000000 & 0.0000000000000000 & 0.0000000000000000 & $-5.44788567006 \mathrm{E}-011$ \\
\hline & 9 & 0.0000000000000000 & 0.0000000000000000 & 0.0000000000000000 & 0.0000000000000000 & $-5.04422875038 \mathrm{E}-011$ \\
\hline & 10 & 0.0000000000000000 & -0.7368421052631556 & 0.0000000000000000 & 0.0000000000000000 & 0.0000000000000000 \\
\hline & 11 & 0.0000000000000000 & 0.5939849624060100 & 1.0000000000121159 & 0.9999999999433177 & 1.0000000000172573 \\
\hline & 12 & -1.9285714287693543 & 0.0000000000000000 & -0.9999999999521777 & -1.0000000000323539 & -1.0000000000486668 \\
\hline & 13 & 0.0000000000000000 & 0.0000000000000000 & 1.0000000000534601 & 0.9999999998479339 & 1.0000000000138589 \\
\hline & 14 & 0.0000000000000000 & -1.3383458646616542 & -0.9999999999801451 & -1.0000000000111520 & -0.9999999999934237 \\
\hline & 15 & 0.0000000000000000 & -0.1879699248120331 & 0.0000000000000000 & 0.0000000000000000 & 0.0000000000000000 \\
\hline & 16 & 0.0000000000000000 & 0.0000000000000000 & 0.0000000000000000 & 0.0000000000000000 & $-1.35910310537 \mathrm{E}-011$ \\
\hline & 17 & 0.0000000000000000 & 0.0000000000000000 & 0.0000000000000000 & 0.0000000000000000 & 0.0000000000000000 \\
\hline & 18 & 0.0000000000000000 & 0.0000000000000000 & 0.0000000000000000 & $3.31471490925 \mathrm{E}-012$ & 0.0000000000000000 \\
\hline & 19 & 0.0000000000000000 & 0.0000000000000000 & 0.0000000000000000 & 0.0000000000000000 & 0.0000000000000000 \\
\hline & 20 & 0.0000000000000000 & 0.0000000000000000 & 0.0000000000000000 & 0.0000000000000000 & 0.0000000000000000 \\
\hline & 21 & 0.0000000000000000 & 0.1435479931732104 & 0.0000000000000000 & 0.0000000000000000 & 0.0000000000000000 \\
\hline & 22 & 0.0000000000000000 & 0.0000000000000000 & 0.0000000000000000 & 0.0000000000000000 & 0.0000000000000000 \\
\hline & 23 & 0.0000000000000000 & -0.4731767182376145 & 0.0000000000000000 & 0.0000000000000000 & 0.0000000000000000 \\
\hline & 24 & 0.0000000000000000 & 0.0000000000000000 & 0.0000000000000000 & 0.0000000000000000 & 0.0000000000000000 \\
\hline & 25 & 0.0000000000000000 & 0.0000000000000000 & 0.0000000000000000 & 0.0000000000000000 & 0.0000000000000000 \\
\hline & 26 & 0.0000000000000000 & 0.0000000000000000 & 0.0000000000000000 & 0.0000000000000000 & 0.0000000000000000 \\
\hline & 27 & $-3.78807204408 \mathrm{E}-002$ & 0.0000000000000000 & 0.0000000000000000 & 0.0000000000000000 & 0.0000000000000000 \\
\hline & 28 & 0.0000000000000000 & 0.0000000000000000 & 0.0000000000000000 & $-2.16568049869 \mathrm{E}-013$ & 0.0000000000000000 \\
\hline & 29 & 0.0000000000000000 & 0.0000000000000000 & 0.0000000000000000 & 0.0000000000000000 & 0.0000000000000000 \\
\hline & 30 & $-3.78807204407 \mathrm{E}-002$ & 0.0000000000000000 & 0.0000000000000000 & 0.0000000000000000 & 0.0000000000000000 \\
\hline & 31 & -0.3030457635112963 & -1.4514297087513373 & 0.0000000000000000 & 0.0000000000000000 & 0.0000000000000000 \\
\hline & 32 & 0.0000000000000000 & 0.0000000000000000 & 0.0000000000000000 & 0.0000000000000000 & 0.0000000000000000 \\
\hline
\end{tabular}

Tabela 4.2: Coeficientes recuperados considerando $m \in\{k, 1.5 k, 2 k, 2.5 k, 3 k\}$ amostras e uma matriz de medição $\Phi$ do tipo BPBD com $L=2$.

Já nos casos $L=2$ e $L=3$ (Figuras 4.3 e 4.4, respectivamente) o sinal original foi também recuperado para $m=1.5 k$ e $m=2 k$ amostras. Este resultado empírico está de alguma forma de acordo com a teoria que sugere que, com alta probabilidade, o sinal original esparso deveria ser recuperado utilizando a partir de $m \gtrsim k \log _{10} n \approx 1.5 k$ amostras. Considerando que para $L=2$ e $L=3$ as imagens recuperadas são similares para todos os valores de $m$ e lembrando que a complexidade de operar com a matriz de amostragem $\Phi$ do tipo BPBD com parâmetro $L$ é $O(L n)$, fixamos para os experimentos das seções seguintes $L=2$.

\subsection{Experimentos sem ruido}

Nesta seção, abordaremos problemas de programação linear de tipo (4.3) provenientes de considerar ambientes em que o sinal é amostrado sem ruido. Em todos os casos utilizaremos a matriz de medição $\Phi$ do tipo BPBD com $L=2$. Compararemos o desempenho de CPLEX (IBM ILOG AMPL/CPLEX 12.1.0) [9] e Algencan 2.4.0 [3, 4] para resolver os problemas de programação linear e analisaremos a qualidade do sinal recuperado em função do número de amostras utilizado.

Para resolver o problema de programação linear (4.3), o CPLEX precisa da matriz $\Theta=\Phi \Psi^{T}$. De fato, precisa da matriz $[\Theta-\Theta]$, que aparece de forma explícita ao escrever a restrição 


\begin{tabular}{|c|c|c|c|c|c|c|}
\hline & \multirow{2}{*}{$\mathrm{i}$} & \multicolumn{5}{|c|}{ Número de amostras } \\
\hline & & $m=k$ & $m=1.5 k$ & $m=2 k$ & $m=2.5$ & $m=3 k$ \\
\hline \multirow{32}{*}{ *co } & 1 & 4.2426406871192945 & 4.2426406871192874 & 4.2426406871192865 & 4.2426406871192874 & 4.2426406871192919 \\
\hline & 2 & 0.0000000000000000 & 0.0000000000000000 & 0.0000000000000000 & 0.0000000000000000 & 0.0000000000000000 \\
\hline & 3 & 0.0000000000000000 & 0.0000000000000000 & 1.4999999999999984 & 0.9999999999998134 & 0.9999999999994673 \\
\hline & 4 & 0.0000000000000000 & -0.6315789473684206 & 0.0000000000000000 & -1.0000000000000719 & -0.9999999999994371 \\
\hline & 5 & 0.0000000000000000 & 0.0000000000000000 & 0.0000000000000000 & 0.0000000000000000 & 0.0000000000000000 \\
\hline & 6 & 0.0000000000000000 & 0.0000000000000000 & 0.0000000000000000 & 0.0000000000000000 & 0.0000000000000000 \\
\hline & 7 & 0.0000000000000000 & 0.0000000000000000 & 0.0000000000000000 & 0.0000000000000000 & 0.0000000000000000 \\
\hline & 8 & 0.0000000000000000 & 0.0000000000000000 & 0.0000000000000000 & 0.0000000000000000 & 0.0000000000000000 \\
\hline & 9 & 0.0000000000000000 & 0.0000000000000000 & 0.0000000000000000 & 0.0000000000000000 & 0.0000000000000000 \\
\hline & 10 & 0.0000000000000000 & 0.0000000000000000 & 0.0000000000000000 & 0.0000000000000000 & 0.0000000000000000 \\
\hline & 11 & 0.0000000000000000 & 1.7631578947368427 & 1.0000000000000023 & 0.9999999999999608 & 1.0000000000001077 \\
\hline & 12 & 0.0000000000000000 & -0.9473684210526305 & -0.9999999999999250 & -1.0000000000000524 & -1.0000000000000813 \\
\hline & 13 & 0.4999999999978739 & 1.4473684210526330 & 1.0000000000000064 & 0.9999999999999766 & 0.9999999999990230 \\
\hline & 14 & 0.0000000000000000 & -0.3157894736842113 & -0.9999999999999999 & -1.0000000000000930 & -0.9999999999985322 \\
\hline & 15 & 0.0000000000000000 & 0.0000000000000000 & 0.0000000000000000 & 0.0000000000000000 & 0.0000000000000000 \\
\hline & 16 & 0.0000000000000000 & 0.0000000000000000 & 0.0000000000000000 & 0.0000000000000000 & 0.0000000000000000 \\
\hline & 17 & 0.0000000000000000 & $-1.86080731892 \mathrm{E}-002$ & 0.0000000000000000 & 0.0000000000000000 & 0.0000000000000000 \\
\hline & 18 & 0.0000000000000000 & 0.0000000000000000 & 0.0000000000000000 & 0.0000000000000000 & 0.0000000000000000 \\
\hline & 19 & 0.3535533905940678 & 0.0000000000000000 & 0.0000000000000000 & 0.0000000000000000 & 0.0000000000000000 \\
\hline & 20 & 0.0000000000000000 & 0.0000000000000000 & 0.0000000000000000 & 0.0000000000000000 & 0.0000000000000000 \\
\hline & 21 & -0.3535533905923411 & 0.0000000000000000 & $8.83883476483 \mathrm{E}-022$ & 0.0000000000000000 & 0.0000000000000000 \\
\hline & 22 & 0.0000000000000000 & 0.0000000000000000 & 0.0000000000000000 & 0.0000000000000000 & 0.0000000000000000 \\
\hline & 23 & 0.0000000000000000 & 0.0000000000000000 & 0.0000000000000000 & 0.0000000000000000 & 0.0000000000000000 \\
\hline & 24 & 0.3535533905940678 & 0.0000000000000000 & 0.0000000000000000 & 0.0000000000000000 & 0.0000000000000000 \\
\hline & 25 & 0.0000000000000000 & 0.0000000000000000 & 0.0000000000000000 & 0.0000000000000000 & 0.0000000000000000 \\
\hline & 26 & 0.0000000000000000 & 0.0000000000000000 & 0.0000000000000000 & 0.0000000000000000 & 0.0000000000000000 \\
\hline & 27 & 0.0000000000000000 & 0.0000000000000000 & 0.0000000000000000 & 0.0000000000000000 & 0.0000000000000000 \\
\hline & 28 & 0.0000000000000000 & 0.0000000000000000 & 0.0000000000000000 & 0.0000000000000000 & 0.0000000000000000 \\
\hline & 29 & 0.0000000000000000 & 0.0000000000000000 & 0.0000000000000000 & 0.0000000000000000 & 0.0000000000000000 \\
\hline & 30 & 0.0000000000000000 & 0.2791210978367932 & 0.0000000000000000 & 0.0000000000000000 & 0.0000000000000000 \\
\hline & 31 & 0.0000000000000000 & 0.2977291710259142 & 0.0000000000000000 & 0.0000000000000000 & 0.0000000000000000 \\
\hline & 32 & 0.0000000000000000 & 0.0000000000000000 & 0.0000000000000000 & 0.0000000000000000 & 0.0000000000000000 \\
\hline
\end{tabular}

Tabela 4.3: Coeficientes recuperados considerando $m \in\{k, 1.5 k, 2 k, 2.5 k, 3 k\}$ amostras e uma matriz de medição $\Phi$ do tipo BPBD com $L=3$.

$\Theta(u-v)=y$ no formato padrão. No caso de CS, isto é uma limitação para o uso do CPLEX, já que calcular e armazenar a matriz $\Theta$ é custoso. Por outro lado, métodos que utilizem apenas o produto matriz-vetor $\Theta \omega$, para um vetor arbitrário $\omega \in \mathbb{R}^{n}$ dado, poderão resolver o problema de forma mais eficiente. Este é o caso da versão 2.4.0 de Algencan que, modificado ad-hoc para ser utilizado neste trabalho, incorpora uma opção que permite que o usuário implemente apenas três subrotinas para calcular: (a) a função objetivo e as restrições; (b) o produto do Jacobiano das restrições (ou sua transposta) por um vetor arbitrário dado; e (c) o produto da Hessiana do Lagrangiano (matriz nula no caso do problema (4.3)) por um vetor arbitrário dado. Nos experimentos numéricos que seguem utilizamos Algencan 2.4.0 implementando estas três subrotinas, o que corresponde com uma versão de Algencan livre de matrizes (livre de computar, armazenar e fatorizar matrizes). Utilizamos todos os parâmetros default de Algencan a menos de dois: (i) desabilitamos o escalamento da função objetivo e as restrições com a palavra chave OBJECTIVE-AND-CONSTRAINTS-SCALING-AVOIDED, já que faze-lo implicaria o cálculo individual do gradiente de cada restrição, quer dizer, da matriz de restrições $\Theta$; e (ii) forçamos o uso do produto verdadeiro da Hessiana do Lagrangiano aumentado vezes um vetor usando a palavra chave TRUE-HESSIAN-PRODUCT-IN-CG. Basta dizer que esta é a decisão adequada a ser tomada neste caso mas que Algencan utiliza, de forma arbitrária, uma aproximação da Hessiana do Lagrangiano aumentado [11] quando o número de variáveis é maior do que 20,000. Para mais 


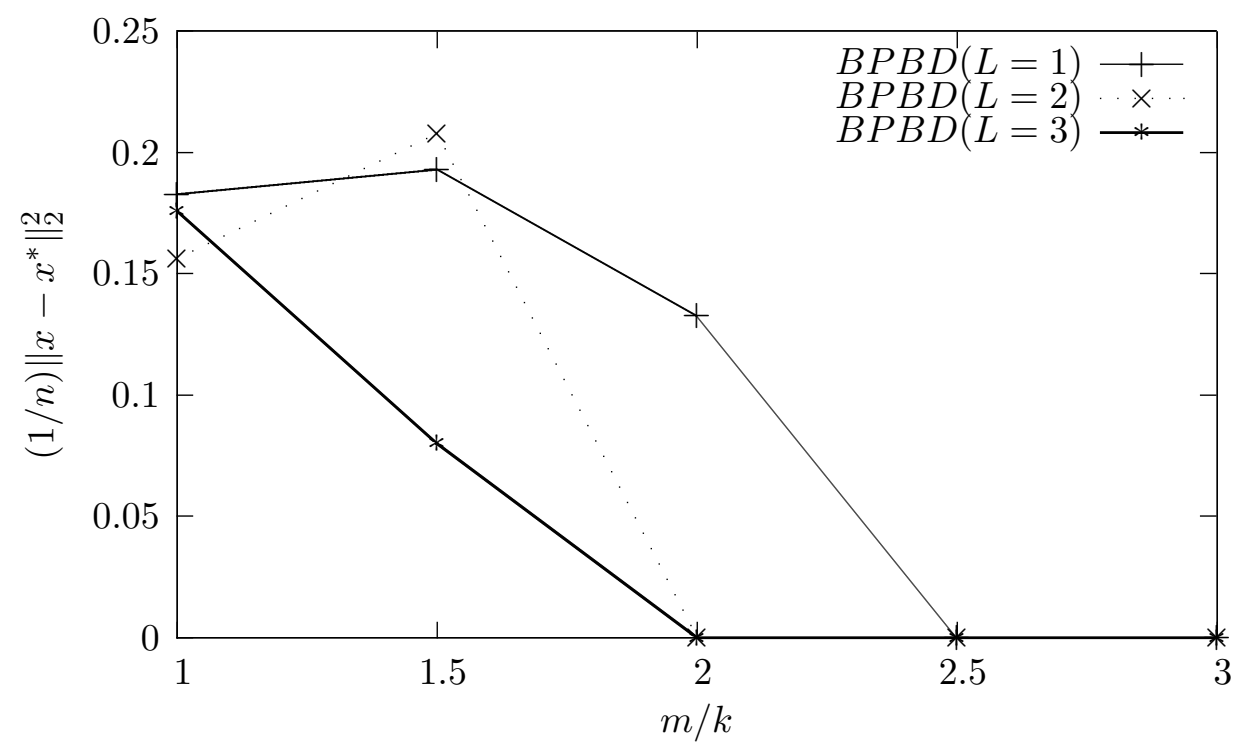

Figura 4.5: Comparação das soluções do problema (2.19) para diferentes valores de $m$ e $L$.

detalhes veja [10]. No caso do CPLEX utilizamos todos os parâmetros default.

Para os experimentos numéricos desta seção consideramos as imagens Phantom e Camera Man, ambas de $256 \times 256$ pixels, ilustradas na Figura 4.6. A imagem Phantom pode ser gerada com a função phantom [61] do Octave [60] enquanto que a imagem Camera Man está amplamente disponível na web (veja, por exemplo, [58]). A partir delas, utilizando a função imresize [59] do Octave, geramos versões modificadas destas imagens com $32 \times 32,64 \times 64$ e $128 \times 128$ pixels. Daqui em diante denominaremos estas imagens de Phantom 32, Phantom 64, Phantom 128 e Phantom 256, respectivamente, e, analogamente, para o caso da imagem Camera Man.

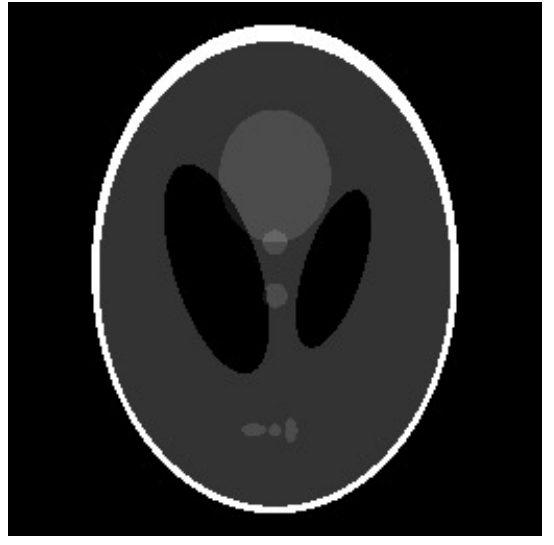

(a) Phantom

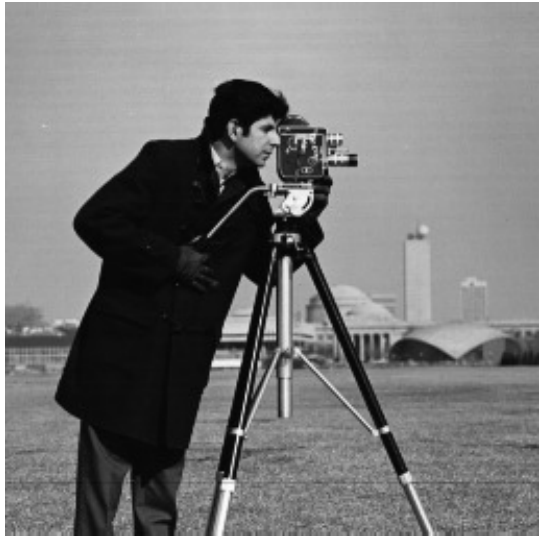

(b) Camera Man

Figura 4.6: Imagens teste.

Para cada uma das oito imagens (imagens Phantom e Camera Man com $32 \times 32,64 \times 64,128 \times 128$ ou $256 \times 256$ pixels) consideramos problemas com $m=\alpha n$ amostras e $\alpha \in\{0.1,0.2, \ldots, 0.9\}$. A Tabela 4.4 apresenta o desempenho de Algencan e CPLEX na resolução destes 72 problemas de 
programação linear. Na Tabela 4.4, $n$ é a quantidade de pixels da imagem considerada (o número de variáveis do problema de programação linear é $2 n$ ) e $\alpha$ é tal que o número de amostras é dado por $m=\alpha n$. Na coluna "Algencan" apresenta-se o tempo de CPU em segundos utilizado por Algencan para resolver o problema. Na coluna "CPLEX" aparecem o tempo de CPU "pre" (preprocessamento) utilizado para gerar a matriz $\Theta$ e o tempo de CPU "sol" (solução) utilizado pelo CPLEX para resolver o problema. O símbolo "-" indica que o tempo de CPU excedeu o limite máximo estipulado em 2.5 horas (8,800 segundos). Em todos os problemas em que Algencan e CPLEX terminaram sua execução dentro do limite de tempo de CPU estabelecido, os valores ótimos encontrados por ambos métodos coincidem até a sexta casa decimal e são apresentados na coluna $\|s\|_{1}$. A análise dos tempos de CPU apresentados na Tabela 4.4 mostra, como já era esperado, que o CPLEX é mais eficiente nos problemas menores enquanto que a versão livre de matriz de Algencan é preferível para resolver os problemas maiores.

\begin{tabular}{|c|c|c|c|c|c|c|c|c|c|c|}
\hline \multirow{3}{*}{$n$} & \multirow{3}{*}{$\alpha$} & \multirow{3}{*}{$m$} & \multicolumn{4}{|c|}{ Imagem Phantom } & \multicolumn{4}{|c|}{ Imagem Camera Man } \\
\hline & & & \multirow{2}{*}{ Algencan } & \multicolumn{2}{|c|}{ CPLEX } & \multirow{2}{*}{$\|s\|_{1}$} & \multirow{2}{*}{ Algencan } & \multicolumn{2}{|c|}{ CPLEX } & \multirow{2}{*}{$\|s\|_{1}$} \\
\hline & & & & pre & sol & & & pre & sol & \\
\hline \multirow[b]{2}{*}{ 崌 } & 0.1 & 102 & 0.27 & 0.02 & 0.03 & 37.224238 & 0.26 & 0.01 & 0.03 & 53.799529 \\
\hline & 0.2 & 204 & 0.70 & 0.01 & 0.07 & 54.672388 & 0.73 & 0.01 & 0.08 & 72.391219 \\
\hline ஸे & 0.3 & 307 & 1.07 & 0.02 & 0.15 & 74.142265 & 1.27 & 0.01 & 0.16 & 90.413290 \\
\hline$\times$ & 0.4 & 409 & 1.16 & 0.02 & 0.26 & 85.085042 & 1.12 & 0.02 & 0.26 & 102.872796 \\
\hline กิ & 0.5 & 512 & 1.43 & 0.02 & 0.41 & 95.254448 & 1.92 & 0.01 & 0.37 & 114.122068 \\
\hline$\|$ & 0.6 & 614 & 2.24 & 0.02 & 0.60 & 103.177364 & 3.00 & 0.02 & 0.43 & 122.522672 \\
\hline \multirow{3}{*}{$\begin{array}{l}\stackrel{\sim}{N} \\
\stackrel{-}{-}\end{array}$} & 0.7 & 716 & 3.14 & 0.02 & 0.56 & 107.098199 & 3.31 & 0.02 & 0.55 & 129.208704 \\
\hline & 0.8 & 819 & 0.12 & 0.02 & 0.35 & 108.184847 & 3.48 & 0.02 & 0.57 & 132.650227 \\
\hline & 0.9 & 921 & 0.06 & 0.02 & 0.22 & 108.184847 & 0.64 & 0.02 & 0.45 & 133.549329 \\
\hline \multirow{2}{*}{ 希 } & 0.1 & 409 & 6.50 & 0.20 & 0.86 & 134.743520 & 7.26 & 0.18 & 0.83 & 176.148837 \\
\hline & 0.2 & 819 & 12.41 & 0.22 & 3.52 & 216.518599 & 12.54 & 0.19 & 3.54 & 246.466116 \\
\hline है & 0.3 & 1228 & 21.75 & 0.26 & 8.42 & 269.782353 & 25.36 & 0.22 & 9.31 & 303.415697 \\
\hline$\times$ & 0.4 & 1638 & 32.55 & 0.28 & 15.55 & 311.808579 & 30.95 & 0.25 & 22.08 & 345.632535 \\
\hline రే & 0.5 & 2048 & 34.17 & 0.24 & 27.32 & 338.869225 & 39.96 & 0.21 & 35.07 & 379.151367 \\
\hline$\|$ & 0.6 & 2457 & 1.23 & 0.31 & 27.32 & 338.869225 & 48.99 & 0.27 & 42.83 & 401.182541 \\
\hline$\stackrel{2}{\mathscr{2}}$ & 0.7 & 2867 & 0.92 & 0.35 & 18.24 & 348.912247 & 66.26 & 0.32 & 56.99 & 413.589578 \\
\hline \multirow{2}{*}{ fi } & 0.8 & 3276 & 0.30 & 0.39 & 8.95 & 348.912247 & 1.37 & 0.34 & 34.00 & 414.571902 \\
\hline & 0.9 & 3686 & 0.26 & 0.42 & 4.98 & 348.912247 & 0.96 & 0.36 & 13.84 & 414.571902 \\
\hline 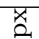 & 0.1 & 1638 & 88.17 & 3.45 & 58.58 & 523.844242 & 66.19 & 3.24 & 66.70 & 600.741653 \\
\hline$\infty$ & 0.2 & 3276 & 271.54 & 3.71 & 431.32 & 796.145496 & 210.43 & 3.55 & 480.06 & 864.182351 \\
\hline$\stackrel{N}{-1}$ & 0.3 & 4915 & 375.16 & 4.08 & $1,565.69$ & 967.986054 & 459.33 & 3.91 & $1,488.89$ & 1042.837885 \\
\hline$\times$ & 0.4 & 6553 & 610.23 & 4.45 & $3,265.81$ & 1051.457258 & 588.99 & 4.27 & $3,838.88$ & 1182.478010 \\
\hline$\stackrel{\infty}{\sim}$ & 0.5 & 8192 & 2.23 & 3.89 & $2,510.99$ & 1063.602312 & 618.20 & 3.79 & $4,473.21$ & 1182.478010 \\
\hline$\|$ & 0.6 & 9830 & 1.61 & 4.83 & $1,818.80$ & 1063.602312 & 611.64 & 4.75 & $6,595.57$ & 1283.331897 \\
\hline$\underbrace{+}$ & 0.7 & 11468 & 1.59 & 5.58 & $1,019.23$ & 1063.602312 & 5.03 & 5.50 & $3,536.30$ & 1283.333904 \\
\hline 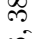 & 0.8 & 13107 & 0.92 & 5.97 & 540.97 & 1063.602312 & 3.31 & 5.85 & $1,849.34$ & 1283.333904 \\
\hline$\stackrel{0}{-1}$ & 0.9 & 14745 & 1.20 & 6.33 & 205.42 & 1063.602312 & 2.19 & 6.24 & 746.42 & 1283.333904 \\
\hline 斑 & 0.1 & 6553 & $4,359.30$ & 58.68 & $7,019.91$ & 2015.043391 & $2,816.86$ & 60.42 & $7,426.60$ & 2182.843851 \\
\hline 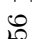 & 0.2 & 13107 & $7,773.51$ & 62.98 & - & 2902.988117 & $3,109.46$ & 65.15 & - & 3022.546589 \\
\hline v & 0.3 & 19660 & $6,258.33$ & 69.16 & - & 3277.830741 & $6,263.21$ & 70.88 & - & 3513.585312 \\
\hline$\times$ & 0.4 & 26214 & 12.41 & 75.08 & - & 3303.274688 & $8,403.79$ & 76.26 & - & 3767.363661 \\
\hline 点 & 0.5 & 32768 & 6.45 & 67.18 & - & 3303.274688 & 15.16 & 67.18 & - & 3768.553034 \\
\hline$\|$ & 0.6 & 39321 & 4.65 & 82.78 & - & 3303.274688 & 16.27 & 81.49 & - & 3768.553034 \\
\hline 0 & 0.7 & 45875 & 5.41 & 94.27 & - & 3303.274688 & 9.37 & 94.03 & - & 3768.553034 \\
\hline 管 & 0.8 & 52428 & 4.84 & 100.29 & - & 3303.274688 & 7.55 & 98.33 & - & 3768.553034 \\
\hline 10 & 0.9 & 58982 & 4.28 & 105.65 & - & 3303.274688 & 7.70 & 104.77 & - & 3768.553034 \\
\hline
\end{tabular}

Tabela 4.4: Desempenho de Algencan e CPLEX para resolver 72 problemas de programação linear associados à recuperação de sinais amostrados sem ruido.

Considerando os resultados obtidos, analisaremos agora a qualidade da imagem recuperada para diferentes valores do números de amostras $m$, utilizando Algencan para resolver os problemas de programação linear correspondentes. Para estes experimentos consideraremos as imagens 
Phantom 256 e Camera Man 256 (ambas com $n=256 \times 256$ pixels) ilustradas na Figura 4.6. A densidade (4.2) da imagem Phantom 256 é 9.7\% enquanto que a densidade da imagem Camera Man 256 é 89.8\%. As Figuras 4.7a e 4.7b ilustram as entradas $s_{i}$ dos coeficientes $s$ das imagens Phantom 256 e Camera Man 256, ordenando-as em ordem decrescente de $\left|s_{i}\right|$, respectivamente. A Figura 4.7a mostra que, como pode ser deduzido da densidade da imagem Phantom 256, o seu vetor $s$ de coeficientes tem apenas $k=6,391$ elementos não nulos. Por outro lado, a Figura 4.7b mostra que o vetor de coeficientes $s$ da imagem Camera Man 256 tem $k=58,852$ elementos não nulos.

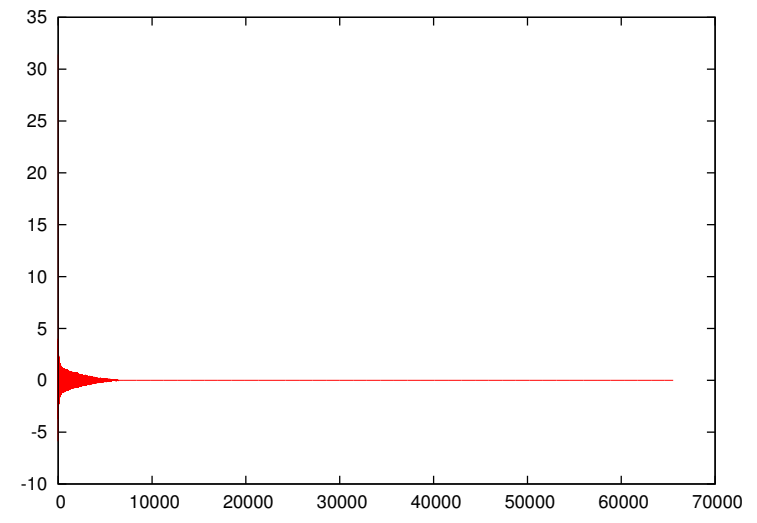

(a)

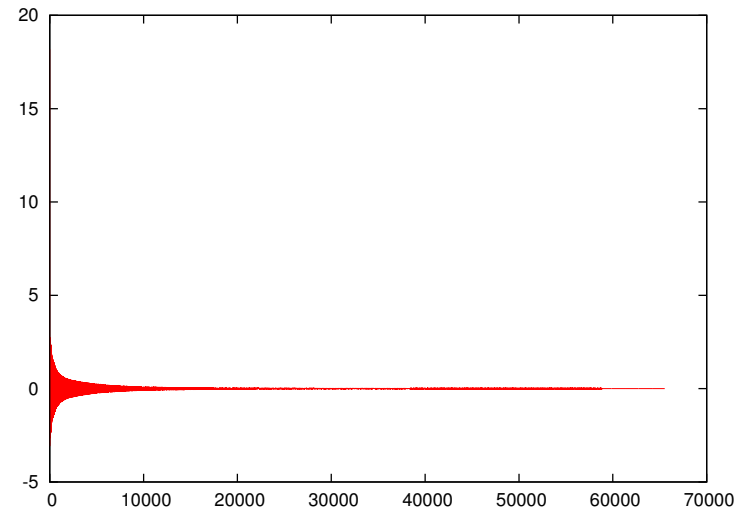

(b)

Figura 4.7: Entradas $s_{i}$ dos coeficientes $s$ das imagens (a) Phantom 256 e (b) Camera Man 256, ordenadas em ordem decrescente de $\left|s_{i}\right|$.

Como as ideias de compressive sensing aplicam-se a sinais esparsos, precisamos "esparsificar" a imagem Camera Man 256 eliminando as entradas menos significativas (valores pequenos de $\left|s_{i}\right|$ ) no seu vetor de coeficientes $s$. Dada uma constante $c>0$, consideraremos as imagens resultantes de eliminar as entradas $s_{i}$ tais que $\left|s_{i}\right| \leq c\|s\|_{\infty}$. As Figuras 4.8a-4.8j ilustram as imagens resultantes de considerar $c=0.0010,0.0009,0.0008, \ldots, 0.0001$, respectivamente. As figuras mostram, para cada imagem, a densidade resultante do processo de "esparcificação". Daqui em diante, no restante da presente seção, consideraremos a imagem Phantom 256 original com densidade 9.7\% e a imagem Camera Man 256 "esparsificada" com $c=0.0010$ e densidade $14.17 \%$ que, com abuso de notação, continuaremos chamando de Camera Man 256.

Tendo selecionado as duas imagens, consideraremos agora os problemas de programação linear relacionados ao problema de recuperar as imagens selecionadas utilizando $m \in\{1.5 k, 2 k, 2.5 k$, $3 k, 3.5 k, 4 k, 4.5 k, 5 k\}$ amostras, onde $k=\|s\|_{0}=6,391$ para a imagem Phantom 256 e $k=$ $\|s\|_{0}=9,786$ para a imagem Camera Man 256 representam a esparsidade do sinal. Os 8 problemas de programação linear (duas imagens e quatro valores possíveis para o número de amostras $m$ ) foram resolvidos utilizando Algencan com os parâmetros já mencionados.

A Tabela 4.5 mostra o desempenho do Algencan ao resolver cada um dos 16 problemas. $\mathrm{Na}$ tabela, $m / k$ é razão entre o número de amostras e a espasidade do sinal, $\|s\|_{1}$ é o valor da função objetivo de (4.3) e $\|y-\Theta s\|_{\infty}$ é a norma infinito da viablidade de (4.3), \# outit, \# innit 


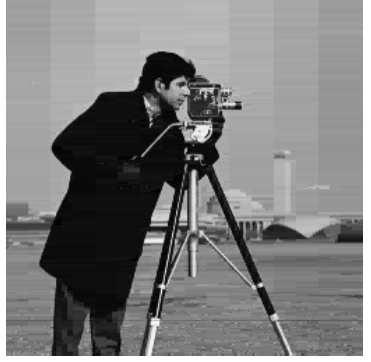

(a) $c=0.0010(14.17 \%)$

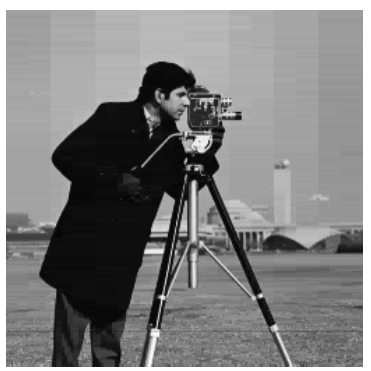

(e) $c=0.0006(19.52 \%)$

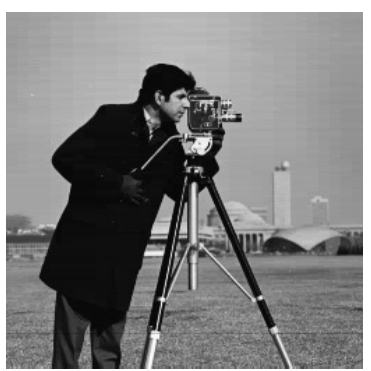

(i) $c=0.0002(34.19 \%)$

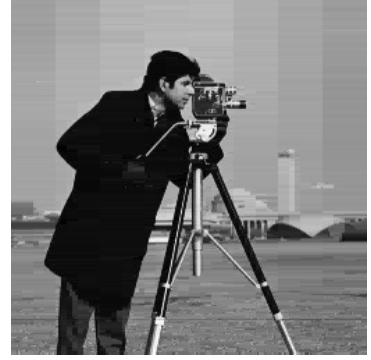

(b) $c=0.0009(15.52 \%)$

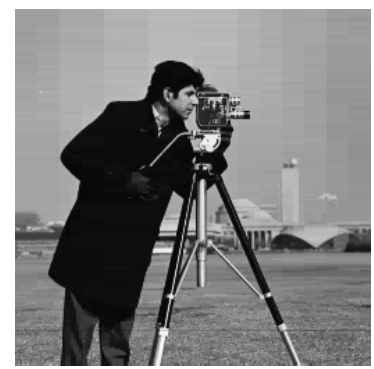

(f) $c=0.0005(21.79 \%)$

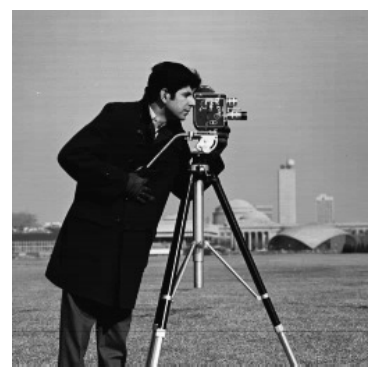

(j) $c=0.0001(47.58 \%)$

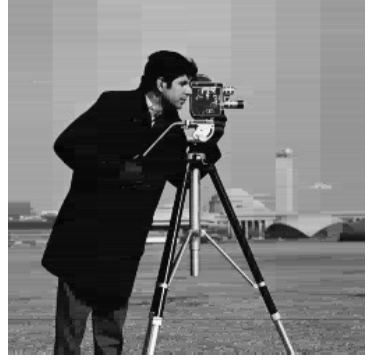

(c) $c=0.0008(16.60 \%)$

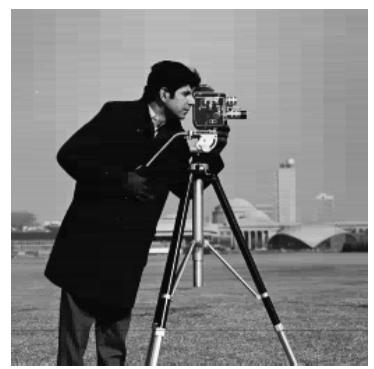

(g) $c=0.0004(24.63 \%)$

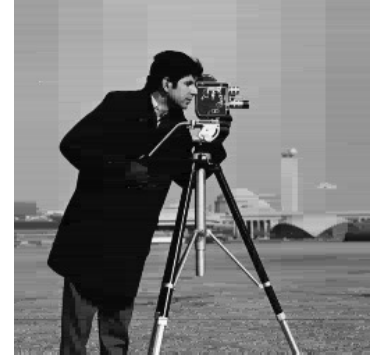

(d) $c=0.0007(17.81 \%)$

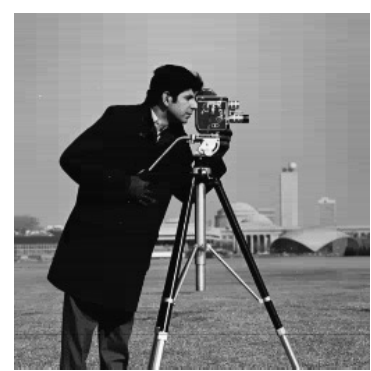

(h) $c=0.0003(28.84 \%)$

Figura 4.8: Imagens resultantes de aplicar o processo de "esparcificação" à imagem Camera Man 256.

e Tempo representam uma medida do custo computacional de resolver o problema e rerem-se ao número de iterações externas e internas de Algencan e ao tempo de $\mathrm{CPu}$ em segundos, respectivamente.

As Figuras 4.9 e 4.10 apresentam as imagens recuperadas. As Figuras 4.11 e 4.12 ilustram a diferença entre a imagem original e as imagens recuperadas. Analisando as imagens recuperadas observamos que, tanto para a imagem Phantom 256 como para a imagem Camera Man 256, as imagens recuperadas são muito parecidas às imagens originais quando $m \in\{2.5 k, 3 k, \ldots, 5 k\}$.

\begin{tabular}{c||c|r|r|r|r||r|r|r|r|r}
\cline { 2 - 10 } \multicolumn{4}{c|}{ Imagem Phantom 256 } & \multicolumn{4}{|c}{ Imagem Camera Man 256 } \\
\hline$m / k$ & $\|s\|_{1}$ & $\|y-\Theta s\|_{\infty}$ & \# outit & \# innit & Tempo & $\|s\|_{1}$ & $\|y-\Theta s\|_{\infty}$ & \# outit & \# innit & Tempo \\
\hline 1.5 & 2488.378252 & $1.90 \mathrm{E}-11$ & 19 & 2163 & 6978.62 & 3151.149480 & $7.50 \mathrm{E}-09$ & 21 & 1291 & 4878.00 \\
2.0 & 2887.744625 & $3.20 \mathrm{E}-09$ & 22 & 1637 & 6395.56 & 3508.192221 & $3.40 \mathrm{E}-09$ & 24 & 2245 & 11495.99 \\
2.5 & 3132.373870 & $9.10 \mathrm{E}-09$ & 21 & 1301 & 3851.97 & 3739.190799 & $5.30 \mathrm{E}-09$ & 23 & 2405 & 7500.96 \\
3.0 & 3265.240770 & $1.40 \mathrm{E}-09$ & 22 & 1451 & 6119.39 & 3768.553034 & $2.40 \mathrm{E}-12$ & 26 & 752 & 32.01 \\
3.5 & 3303.274688 & $5.30 \mathrm{E}-12$ & 16 & 533 & 22.01 & 3768.553034 & $4.30 \mathrm{E}-12$ & 19 & 321 & 11.81 \\
4.0 & 3303.274688 & $1.30 \mathrm{E}-10$ & 16 & 347 & 11.15 & 3768.553034 & $2.60 \mathrm{E}-13$ & 18 & 289 & 12.70 \\
4.5 & 3303.274688 & $6.50 \mathrm{E}-12$ & 17 & 241 & 8.90 & 3768.553034 & $3.80 \mathrm{E}-12$ & 18 & 206 & 9.44 \\
5.0 & 3303.274688 & $6.40 \mathrm{E}-12$ & 15 & 188 & 6.75 & 3768.553034 & $3.00 \mathrm{E}-12$ & 19 & 194 & 9.52 \\
\hline
\end{tabular}

Tabela 4.5: Detalhe do desempenho do Algencan na resolução do problema (4.3) para as imagens Phantom 256 e Camera Man 256 variando o número de amostras $m$. 


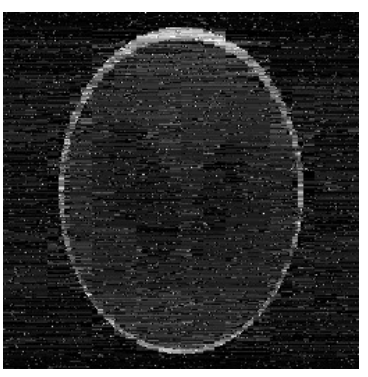

$m=1.5 k$

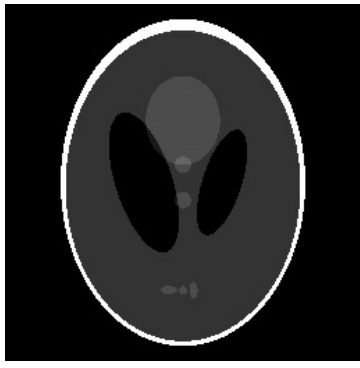

$m=3.5 k$

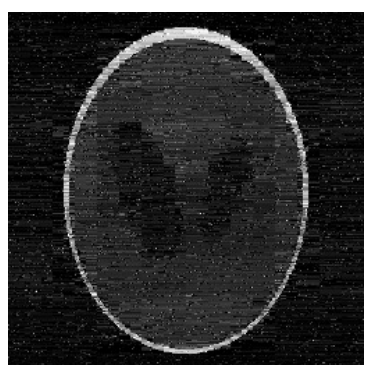

$m=2 k$

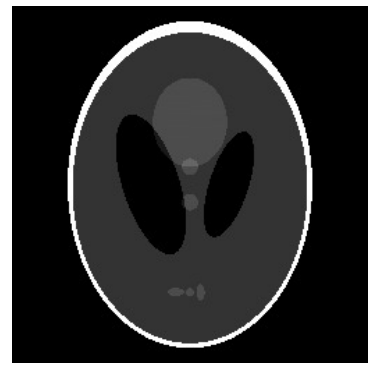

$m=4 k$

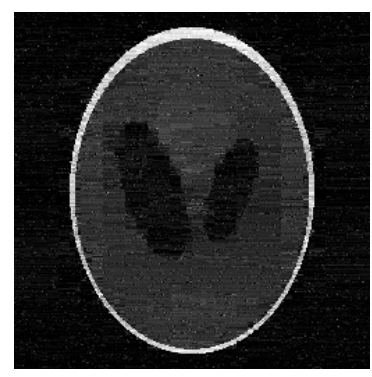

$m=2.5 k$

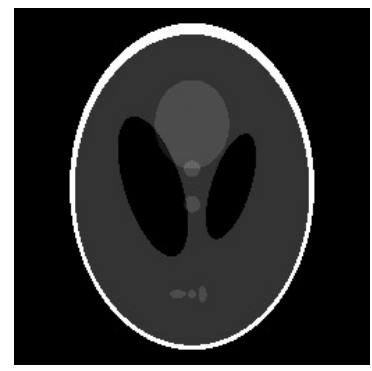

$m=4.5 k$

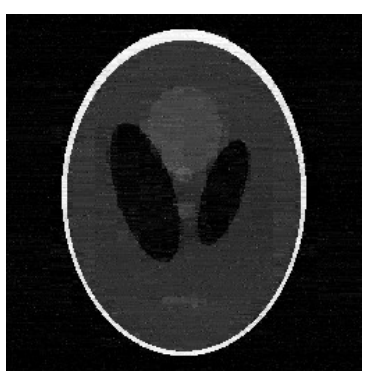

$m=3 k$

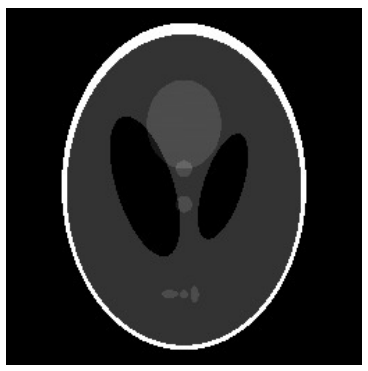

$m=5 k$

Figura 4.9: Imagens recuperadas a partir da solução do problema de programação linear (4.3) para a imagem Phantom 256 e considerando diferentes valores para o número de amostras $m$.

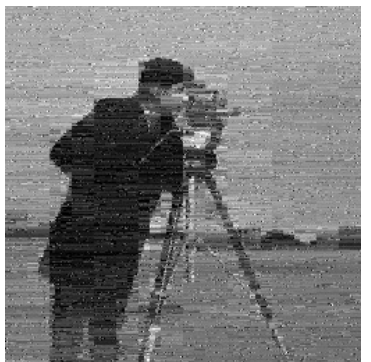

$m=1.5 k$

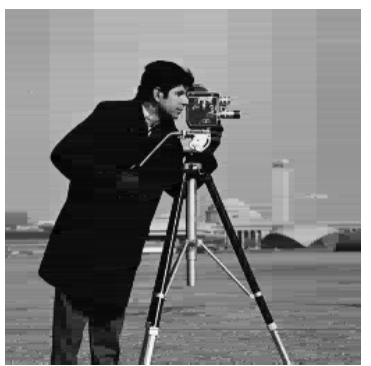

$m=3.5 k$

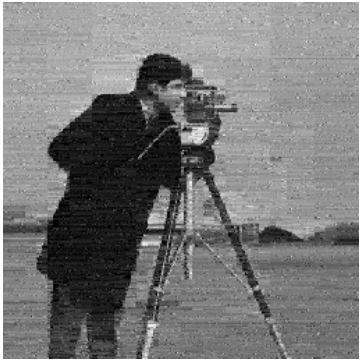

$m=2 k$

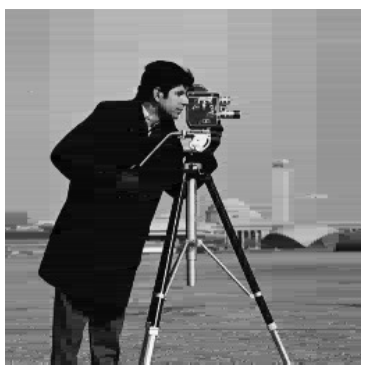

$m=4 k$

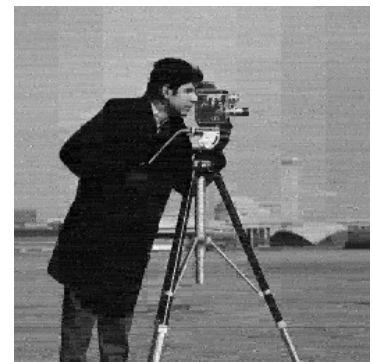

$m=2.5 k$

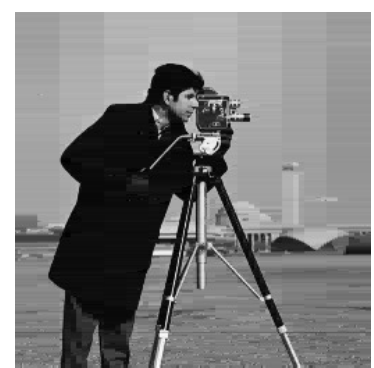

$m=4.5 k$

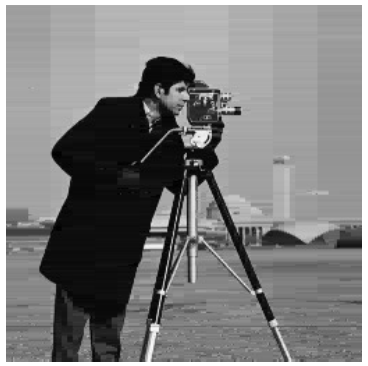

$m=3 k$

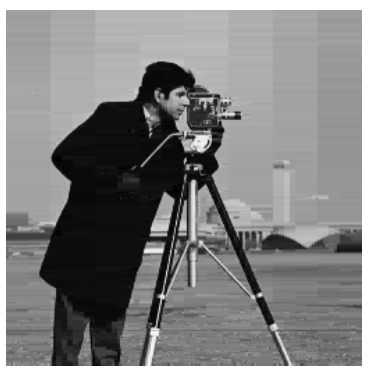

$m=5 k$

Figura 4.10: Imagens recuperadas a partir da solução do problema de programação linear (4.3) para a imagem Camera Man 256 e considerando diferentes valores para o número de amostras $\mathrm{m}$.

\subsection{Experimentos com ruido}

Nesta seção abordaremos a resolução de uma sequência de problemas do tipo (3.3-3.4) para diferentes valores do parâmetro $\gamma \in[0,1]$. Para um valor fixo de $\gamma$, o problema (3.3-3.4) consiste em minimizar uma quadrática convexa sujeita a restrições de caixa. Como já foi mencionado no Capítulo 3, este problema vem de considerar ambientes em que o sinal original é amostrado 


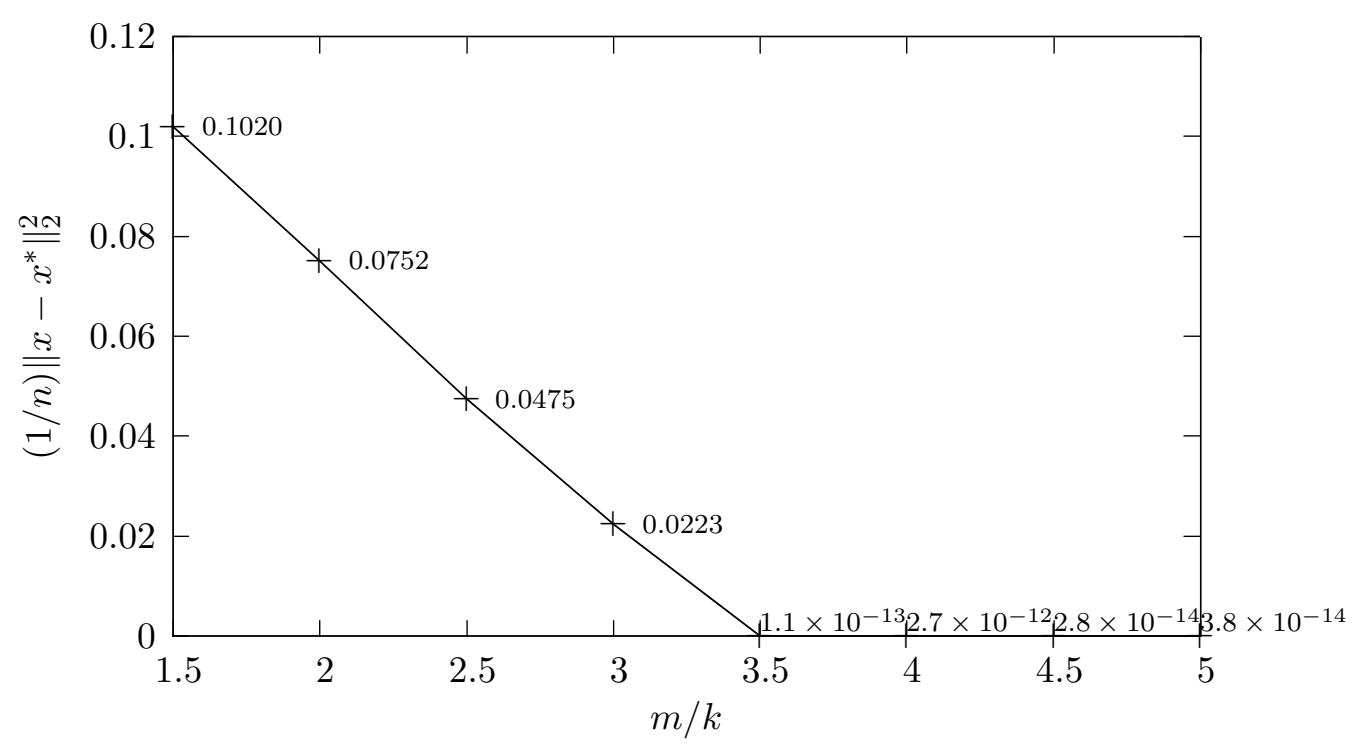

Figura 4.11: Comparação da imagem verdadeira Phantom 256 com a imagem recuperada utilizando a solução do problema de programação linear (4.3) com diferentes valores do número de amostras $m$.

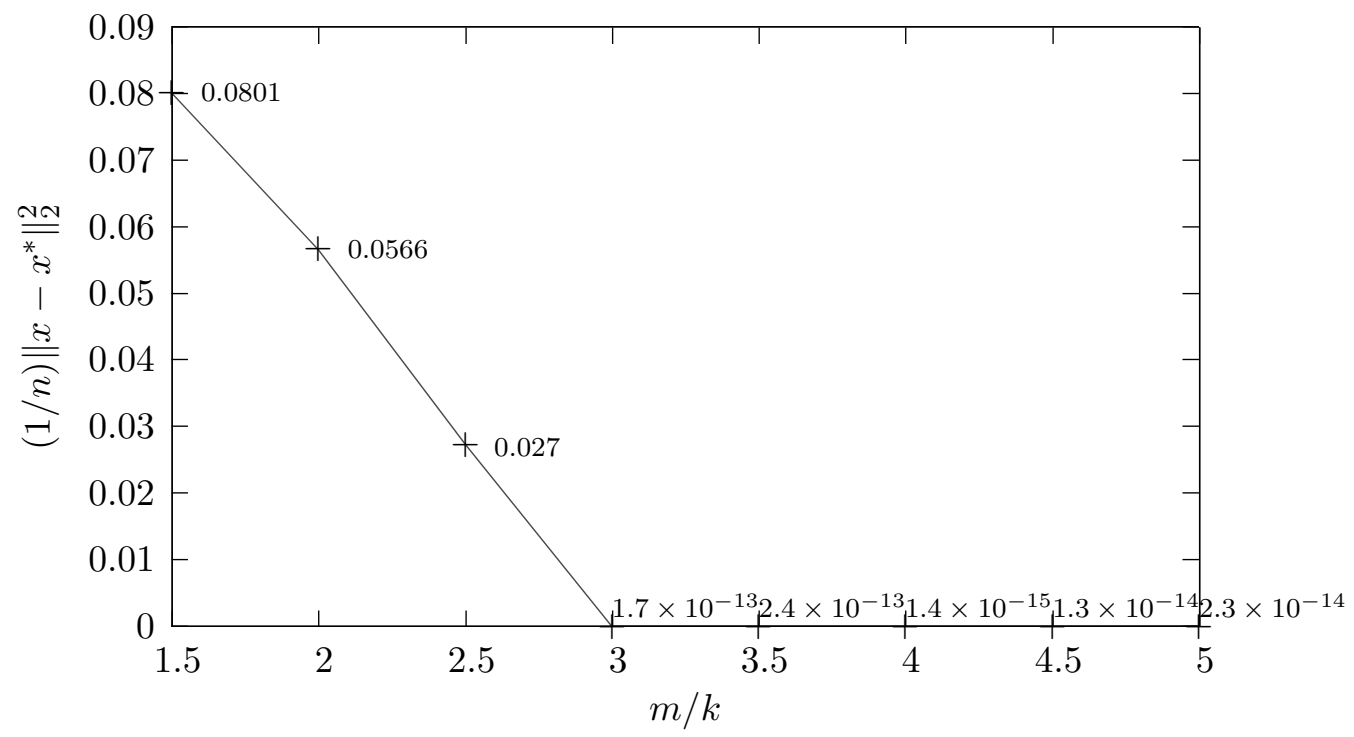

Figura 4.12: Comparação da imagem verdadeira Camera Man 256 com a imagem recuperada utilizando a solução do problema de programação linear (4.3) com diferentes valores do número de amostras $m$.

com ruido. Ao longo desta seção utilizaremos a matriz de medição $\Phi$ do tipo BPBD $\operatorname{com} L=2$. Analisaremos a qualidade do sinal recuperado em função de numero de amostras $m$ e o nível de ruido introduzido.

Para os experimentos numéricos consideraremos primeiramente as imagens Phantom 256 e $C a$ mera Man 256 consideradas na Seção 4.2 (quer dizer, com abuso de notação, continuamos chamando de Camera Man 256 à versão esparsificada da imagem Camera Man 256 original que foi utilizada na seção anterior). Acompanhando o desenvolvimento do trabalho [33], utilizaremos o método do Gradiente Espectral Projetado (SPG) $[12,16]$ com todos seus parâmetros default 
para resolver os problemas do tipo (3.3). Isto quer dizer que utilizaremos $M=10, \lambda_{\min }=10^{-30}$, $\lambda_{\max }=10^{30}, \sigma_{1}=0.1, \sigma_{2}=0.9, \gamma_{\text {arm }}=10^{-4}$ e $\varepsilon=10^{-6}$.

Para introduzir ruido nas amostras $y$ (que denotam amostras sem ruido da forma $y=\Phi x$, onde $x$ é a imagem original) e assim simular um possível erro no instrumento de medição, redefinimos $y$ como

$$
y_{i} \leftarrow y_{i}+\eta_{i}^{y} \times y_{i}, i=1, \ldots, m,
$$

onde $\eta_{i}^{y} \sim \mathcal{N}\left(0, \sigma_{y}^{2}\right)$. Quer dizer, $\eta_{i}^{y}$ segue uma distribuição normal $\mathcal{N}(\cdot, \cdot)$ com média 0 e variança $\sigma_{y}^{2}$, onde $\sigma_{y}>0$ define o nível de ruido considerado nas amostras. De forma, análoga, para introduzir ruido na imagem verdadeira $x$ e assim simular possíveis condições adversas no ambiente, redefinimos $x$ como

$$
x_{i} \leftarrow x_{i}+\eta_{i}^{x} \times x_{i}, i=1, \ldots, m,
$$

onde $\eta_{i}^{x} \sim \mathcal{N}\left(0, \sigma_{x}^{2}\right)$. Nos experimentos a seguir consideraremos ruido tanto no sinal como nas amostras e utilizaremos $\sigma_{x}=\sigma_{y} \in\{0.01,0.02, \ldots, 0.09\}$.

Para comparar o sinal recuperado $x$ com o sinal original $x^{*}$ utilizaremos a medida Peak Signalto-Noise Ratio (PSNR) [23] definida como

$$
\operatorname{PSNR}\left(x, x^{*}\right)=10 \log _{10}\left(\frac{\left\|x^{*}\right\|_{\infty}^{2}}{\frac{1}{n}\left\|x-x^{*}\right\|_{2}^{2}}\right) .
$$

O valor típico para o $\operatorname{PSNR}(\cdot, \cdot)$ fica entre $30 \mathrm{~dB}$ e $50 \mathrm{~dB}$, e valores entre $20 \mathrm{~dB}$ e $25 \mathrm{~dB}$ são considerados aceitáveis. Um valor "alto" do $\operatorname{PSNR}(\cdot, \cdot)$ indica uma boa qualidade da imagem recuperada [23]. A solução $x$ do problema (3.3-3.4) é de fato algo do tipo $x(\gamma)$. Nos experimentos numéricos a seguir, resolveremos uma sequência de problemas (3.3-3.4) para diferentes valores de $\gamma$ e, fazendo uso da solução verdadeira $x^{*}$, apresentaremos como imagem reconstruída, a solução $x(\gamma)$ com maior valor de $\operatorname{PSNR}\left(x(\gamma), x^{*}\right)$.

Foi observado em [33] que problemas do tipo (3.3-3.4) são mais fáceis de resolver quando $\gamma \approx 1$. De fato, para $\gamma=1$ o problema (3.3-3.4) (veja o problema equivalente (3.2)) consiste em achar o $s \in \mathbb{R}^{n}$ com $\|\cdot\|_{1}$ mínima, quer dizer, $s=0$. Isto sugere que uma estratégia válida (do tipo warmstart) para resolver uma sequência de problemas (3.3-3.4) para valores diferentes de $\gamma$ seria começar com $\gamma=1$, ir reduzindo o valor de $\gamma$ até chegar a zero e utilizar como ponto inicial para o problema com um $\gamma$ dado, a solução do problema "anterior". A solução do primeiro problema ( com $\gamma=1$ ) é, como já foi mencionado, $s=0$. Porém, empiricamente, foi também comprovado em [33] que não é necessário resolver problemas do tipo (3.3-3.4) para $\gamma$ variando de 1 até 0. Logo, a nossa estratégia consistirá em variar $\gamma$ de forma decrescente de um certo $\gamma_{\max }$ até um certo $\gamma_{\min }$. A solução inicial para o primeiro problema será dada pela solução do problema

$$
\text { Minimizar }\|s\|_{2} \text { sujeta a } \Theta s=y \text {, }
$$


cuja expressão analítica é dada por

$$
s=\Theta^{T}\left(\Theta \Theta^{T}\right)^{-1} y .
$$

Calculado $s$ como em (4.6), o valor inicial das variáveis $u$ e $v$ do problema (3.3-3.4) é dado por $u=[s]_{+}$e $v=[-s]_{+}$. O valor de $s$ em (4.6) é calculado utilizando Gradientes Conjugados [34] (veja também [45]) para resolver o sistema $\left(\Theta \Theta^{T}\right) \tilde{s}=y$ e depois calculando $s=\Theta^{T} \tilde{s}$.

Na escolha do intervalo $\left[\gamma_{\min }, \gamma_{\max }\right]$ utilizamos como referência o trabalho [15]. Em [15] mostra-se (adaptando-o para a formulação (3.3-3.4)) que se

$$
\gamma \geq \frac{\left\|\Theta^{T} s^{*}\right\|_{\infty}}{1+\left\|\Theta^{T} s^{*}\right\|_{\infty}}
$$

onde $s^{*}$ são os coeficientes da imagem verdadeira $x^{*}$, quer dizer $s^{*}=\Psi x^{*}$, então $s=0$ (ou a sua representação equivalente nas variáveis $u$ e $v$ ) é a solução única do problema (3.3-3.4). Nos experimentos que apresentaremos a seguir, consideraremos $\gamma_{\max }=0.2, \gamma_{\min }=10^{-4}, \gamma_{\text {step }}=10^{-4}$ $\mathrm{e}$

$$
\gamma \leftarrow \gamma_{\max }-(i-1) \gamma_{\text {step }}, i=1, \ldots, 2000
$$

A modo de exemplo, consideremos o problema (3.3-3.4) associado à imagem Phantom 256, com nível de ruido $\sigma_{x}=\sigma_{y}=0.01$ e $m=2 k$ (lembremos que para a imagem Phantom 256 temos $\left.k=\left\|s^{*}\right\|_{0}=6,391\right)$. Se resolvemos de forma independente os 2,000 problemas de otimização provenientes de variar $\gamma$ como sugerido em (4.7), utilizando como ponto inicial o valor de $s$ dado em (4.6), então são necessárias, ao todo, 464,248 iterações do SPG, 492,319 avaliações de função objetivo e 2,577.84 segundos de tempo de CPU. Por outro lado, utilizando a técnica de warmstart são necessárias, ao todo, 75,707 iterações do SPG, 102,502 avaliações de função objetivo e 526.55 segundos de tempo de CPU. A Tabela 4.6 mostra em detalhe estas quantidades para alguns valores de $\gamma$ escolhidos arbitrariamente. Na tabela, $f(s)$ corresponde com a função objetivo do problema (3.3-3.4) (ou, equivalentemente, a função objetivo do problema (3.2)), as colunas $\|s\|_{1}$ e $\frac{1}{2}\|y-\Theta s\|_{2}^{2}$ são auto-explicativas e "\# it", "\# fcnt" e "Tempo" correspondem ao número de iterações do SPG, o número de avaliações de função e o tempo de CPU (em segundos), respectivamente. Em todos os casos o SPG satisfez o critério de parada, já mencionado, dado por $\|\nabla f(s)\|_{\infty} \leq 10^{-6}$. É fácil observar que a estratégia warmstart reduz drásticamente o custo computacional de resolver uma sequência de problemas. No exemplo ilustrado a redução é de, aproximadamente, $80 \%$.

Resumindo, nos experimentos a seguir consideraremos duas imagens (Phantom 256 e Camera Man 256), com nove níveis possíveis de ruido $\left(\sigma_{x}=\sigma_{y} \in\{0.01,0.02, \ldots, 0.09\}\right)$ e, consideraremos ainda um número de amostras $m \in\{2 k, 3 k, 4 k, 5 k\}$ com $k=\left\|s^{*}\right\|_{0}=6,391$ para a imagem Phantom 256 e $k=\left\|s^{*}\right\|_{0}=9,786$ para a imagem Camera Man 256. Temos então 72 problemas diferentes do tipo (3.3-3.4). Note que, para cada um deles, variaremos $\gamma$ como mencionado acima (2,000 valores de $\gamma$ para cada problema), totalizando 144, 000 diferentes problemas de 


\begin{tabular}{|c|c|c|c|c|c|c|c|c|c|c|c|c|}
\hline \multirow[b]{2}{*}{$\gamma$} & \multicolumn{6}{|c|}{ Warmstart } & \multicolumn{6}{|c|}{ Coldstart } \\
\hline & $f(s)$ & $\|s\|_{1}$ & $\frac{1}{2}\|y-\Theta s\|_{2}^{2}$ & \# it & $\#$ fcnt & Tempo & $f(s)$ & $\|s\|_{1}$ & $\frac{1}{2}\|y-\Theta s\|_{2}^{2}$ & $\#$ it & $\#$ fent & Tempo \\
\hline 0.2000 & 259.581909 & 209.931361 & 219.861470 & 37 & 38 & 0.21 & 259.581909 & 209.931361 & 219.861470 & 37 & 38 & 0.21 \\
\hline 0.1000 & $1,011.807852$ & 85.523313 & 178.151762 & 18 & 30 & 0.14 & $1,011.808442$ & 85.523248 & 178.151762 & 123 & 125 & 0.64 \\
\hline 0.0900 & $1,136.957548$ & 72.395117 & 168.205732 & 21 & 33 & 0.16 & $1,136.958367$ & 72.395036 & 168.205732 & 79 & 81 & 0.42 \\
\hline 0.0800 & $1,272.852491$ & 59.781294 & 156.826987 & 28 & 40 & 0.20 & $1,272.852788$ & 59.781268 & 156.826987 & 92 & 94 & 0.49 \\
\hline 0.0700 & $1,419.573794$ & 47.893725 & 143.911331 & 27 & 39 & 0.19 & $1,419.573988$ & 47.893711 & 143.911331 & 92 & 94 & 0.50 \\
\hline 0.0600 & $1,577.894872$ & 36.897847 & 129.357666 & 34 & 47 & 0.24 & $1,577.895057$ & 36.897835 & 129.357666 & 97 & 99 & 0.53 \\
\hline 0.0500 & $1,749.053405$ & 26.946794 & 113.052120 & 37 & 51 & 0.26 & $1,749.053326$ & 26.946798 & 113.052120 & 164 & 169 & 0.88 \\
\hline 0.0400 & $1,934.877011$ & 18.204276 & 94.871184 & 34 & 48 & 0.24 & $1,934.877724$ & 18.204247 & 94.871184 & 134 & 140 & 0.74 \\
\hline 0.0300 & $2,138.361469$ & 10.838438 & 74.664127 & 59 & 74 & 0.40 & $2,138.362695$ & 10.838400 & 74.664127 & 172 & 178 & 0.94 \\
\hline 0.0200 & $2,361.861563$ & 5.125097 & 52.259825 & 86 & 100 & 0.55 & $2,361.862468$ & 5.125078 & 52.259825 & 346 & 351 & 1.87 \\
\hline 0.0100 & $2,609.983429$ & 1.367495 & 27.453653 & 94 & 111 & 0.60 & $2,609.988423$ & 1.367444 & 27.453653 & 644 & 659 & 3.52 \\
\hline 0.0090 & $2,636.296831$ & 1.115134 & 24.831768 & 140 & 158 & 0.87 & $2,636.305730$ & 1.115053 & 24.831768 & 772 & 800 & 4.24 \\
\hline 0.0080 & $2,662.887989$ & 0.887180 & 22.183185 & 153 & 169 & 0.96 & $2,662.900579$ & 0.887079 & 22.183185 & 952 & 984 & 5.21 \\
\hline 0.0070 & $2,689.787245$ & 0.683919 & 19.507642 & 115 & 137 & 0.75 & $2,689.795447$ & 0.683862 & 19.507642 & 1,013 & 1,052 & 5.54 \\
\hline 0.0060 & $2,716.961010$ & 0.506154 & 16.804883 & 197 & 223 & 1.24 & $2,716.970632$ & 0.506096 & 16.804883 & 1,156 & 1,216 & 6.42 \\
\hline 0.0050 & $2,744.486891$ & 0.353936 & 14.074600 & 178 & 198 & 1.13 & $2,744.503548$ & 0.353852 & 14.074600 & 1,419 & 1,471 & 8.04 \\
\hline 0.0040 & $2,772.319798$ & 0.228126 & 11.316492 & 308 & 334 & 1.92 & $2,772.329777$ & 0.228087 & 11.316493 & 1,917 & 2,006 & 10.79 \\
\hline 0.0030 & $2,800.439824$ & 0.129370 & 8.530302 & 254 & 286 & 1.63 & $2,800.457038$ & 0.129319 & 8.530302 & 1,948 & 2,093 & 10.98 \\
\hline 0.0020 & $2,828.907017$ & 0.058040 & 5.715737 & 212 & 251 & 1.38 & $2,828.933237$ & 0.057989 & 5.715739 & 2,969 & 3,222 & 16.81 \\
\hline 0.0010 & $2,857.778994$ & 0.014673 & 2.872437 & 238 & 273 & 1.52 & $2,857.808606$ & 0.014649 & 2.872443 & 4,667 & 5,207 & 26.93 \\
\hline 0.0009 & $2,860.680621$ & 0.011913 & 2.586514 & 277 & 305 & 1.71 & $2,860.718276$ & 0.011884 & 2.586519 & 4,547 & 5,049 & 26.23 \\
\hline 0.0008 & $2,863.596016$ & 0.009430 & 2.300299 & 441 & 490 & 2.72 & $2,863.629041$ & 0.009408 & 2.300304 & 5,735 & 6,526 & 33.46 \\
\hline 0.0007 & $2,866.503537$ & 0.007246 & 2.013793 & 298 & 336 & 1.88 & $2,866.542770$ & 0.007223 & 2.013798 & 7,373 & 8,323 & 42.80 \\
\hline 0.0006 & $2,869.422537$ & 0.005345 & 1.726995 & 445 & 487 & 2.74 & $2,869.461524$ & 0.005327 & 1.727001 & 7,965 & 8,991 & 46.31 \\
\hline 0.0005 & $2,872.346386$ & 0.003734 & 1.439905 & 455 & 508 & 2.78 & $2,872.386342$ & 0.003721 & 1.439913 & 9,064 & 10,251 & 52.74 \\
\hline 0.0004 & $2,875.272257$ & 0.002414 & 1.152522 & 403 & 443 & 2.45 & $2,875.316801$ & 0.002407 & 1.152532 & 9,433 & 10,697 & 55.04 \\
\hline 0.0003 & $2,878.192602$ & 0.001391 & 0.864848 & 260 & 284 & 1.61 & $2,878.257988$ & 0.001382 & 0.864859 & 12,616 & 14,476 & 74.12 \\
\hline 0.0002 & $2,881.135280$ & 0.000654 & 0.576881 & 347 & 381 & 2.14 & $2,881.239946$ & 0.000650 & 0.576897 & 16,158 & 18,294 & 94.19 \\
\hline 0.0001 & $2,884.083823$ & 0.000211 & 0.288619 & 478 & 516 & 2.84 & $2,884.355554$ & 0.000209 & 0.288645 & 26,215 & 29,582 & 153.40 \\
\hline
\end{tabular}

Tabela 4.6: Comparação entre as estratégias warmstart e coldstart para resolver uma sequência de problemas do tipo (3.3-3.4) variando o parâmetro $\gamma$.

otimização que serão resolvidos com o SPG.

A Tabela 4.7 mostra o desempenho do SPG para resolver cada um dos 72 problemas. Na tabela, $m / k$ representa o número de amostras, $\sigma=\sigma_{x}=\sigma_{y}$ é o nível de ruido e $\operatorname{PSNR}(\cdot, \cdot)$ quantifica a qualidade da solução recuperada. Note que a medida $\operatorname{PSNR}(\cdot, \cdot)$ calcula-se utilizando a imagem recuperada $x$ e a imagem verdadeira $x^{*}$. A imagem recuperada $x$ é dada por $x=\Psi^{T} s$, onde $s=u-v$ e $u$ e $v$ representam a solução do problema (3.3-3.4) para um valor de $\gamma$ fixo. Como já mencionado, o $P S N R(\cdot, \cdot)$ apresentado nas tabelas e figuras é o maior dentre todos os obtidos variando $\gamma$. Ainda na Tabela 4.7, "\#it", "\# fcnt" e "Tempo" correspondem ao total de iterações do SPG, total de avaliações de função e tempo de CPU (em segundos) necessarios para resolver cada sequência completa de problemas da forma (3.3-3.4) variando $\gamma$ (utilizando warmstart). Em todos os casos o SPG satisfez o critério de parada.

As Figuras 4.13a-d e 4.13e-h mostram uma comparação das curvas Pareto para valores fixos do número de amostras e variando o nível de ruido nos experimentos com as imagens Phantom 256 e Camera Man 256, respectivamente. As Figuras 4.14 e 4.15 mostram o valor de $\operatorname{PSNR}\left(x, x^{*}\right)$ para as 36 combinações de nível de ruido e número de amostras nos experimentos envolvendo as imagens Phantom 256 e Camera Man 256, respectivamente. Para cada combinação de $\sigma_{x}=\sigma_{y}$ e $m$, como já foi mencionado anteriormente, consideramos como imagem recuperada $x$, dentre todas as 2,000 $x(\gamma)$, aquela com maior valor de $\operatorname{PSNR}\left(x(\gamma), x^{*}\right)$.

Analisando a Figura 4.14, podemos ver que quando $m=3 k$ e $\sigma_{x}=\sigma_{y}=0.01$ o valor do 
Imagem Phantom 256

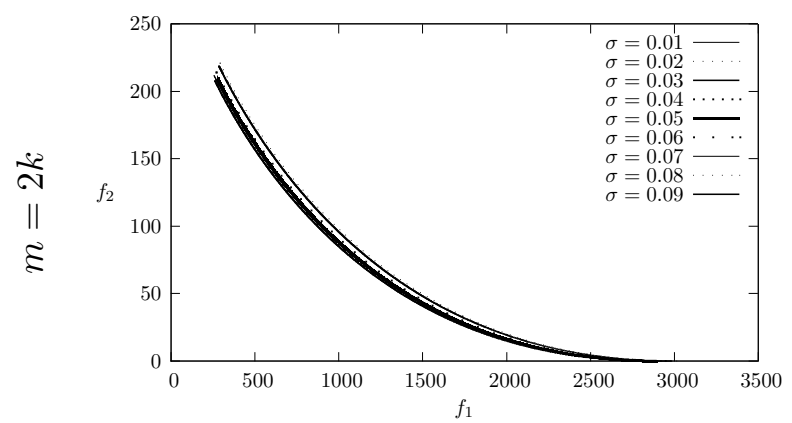

(a)

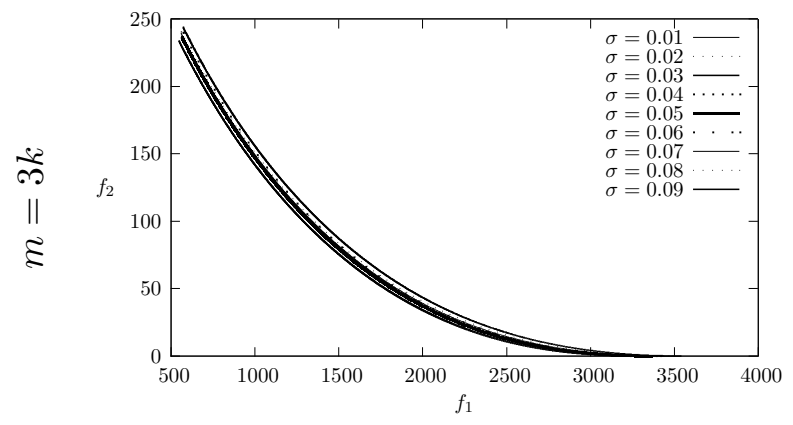

(b)

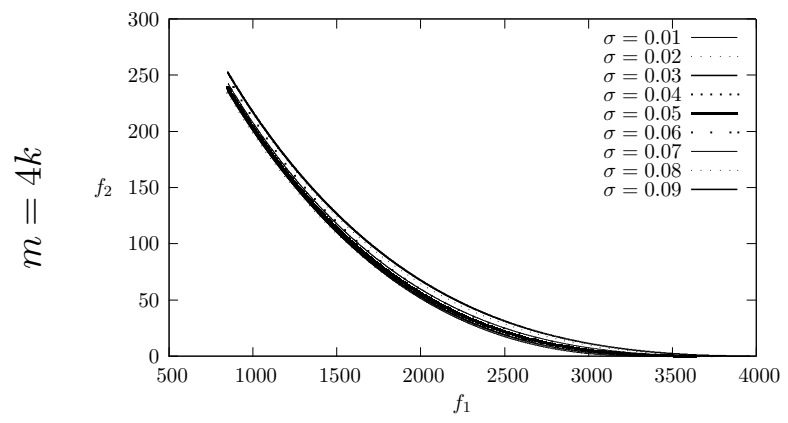

(c)

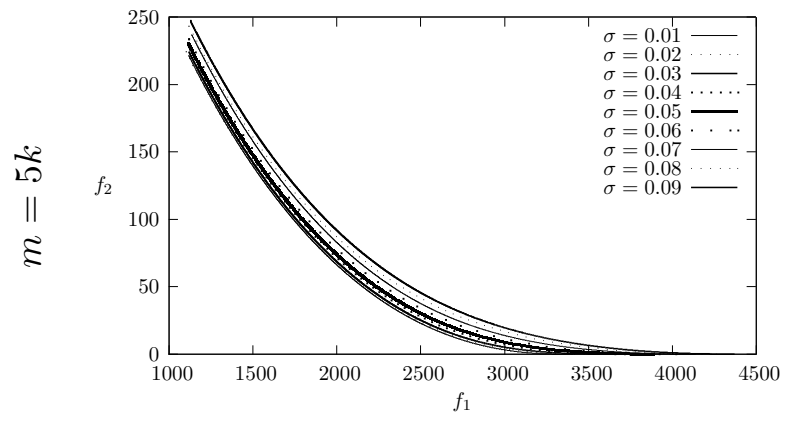

(d)
Imagem Camera Man 256

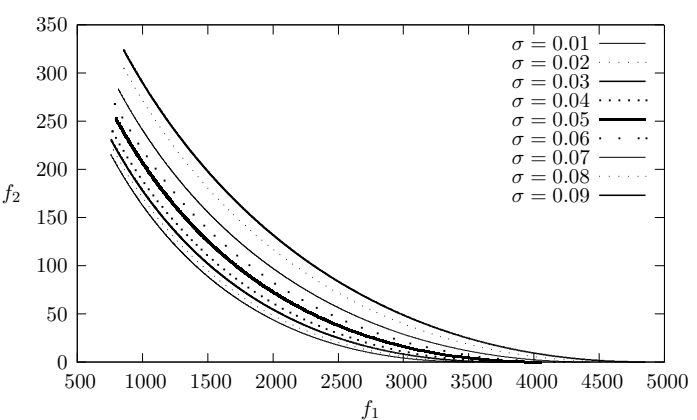

(e)

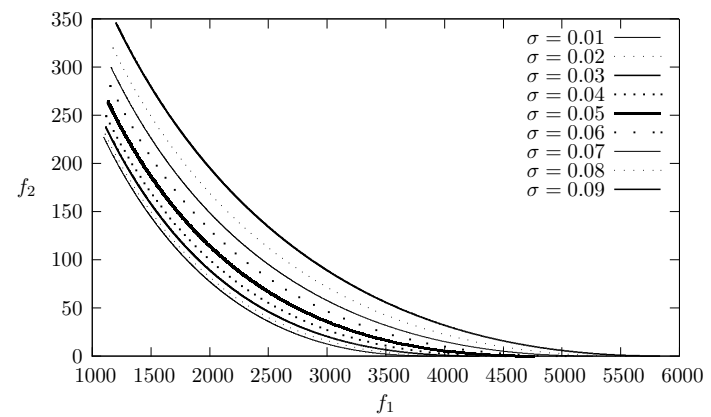

(f)

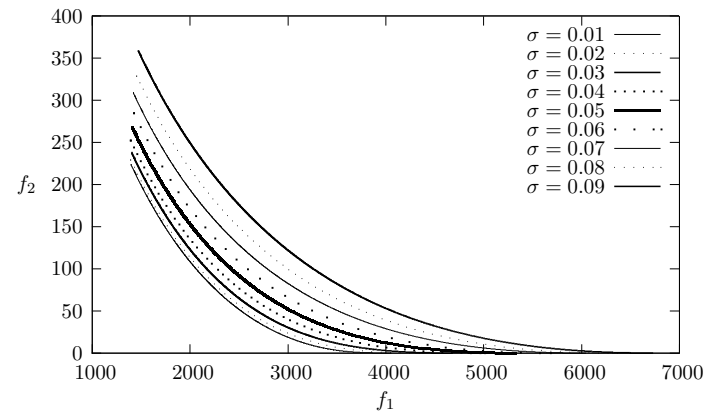

(g)

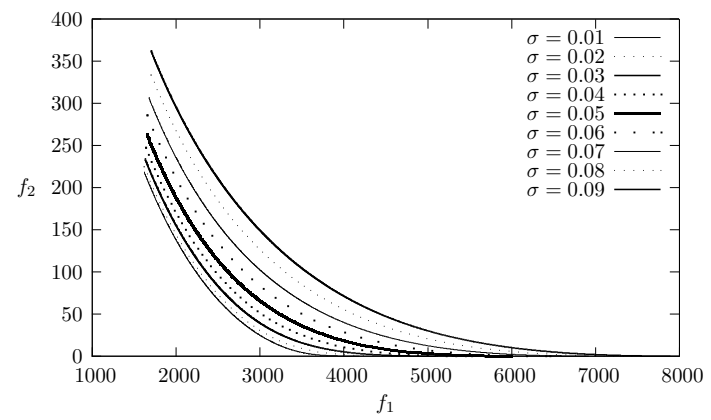

(h)

Figura 4.13: Comportamento das curvas Pareto do problema (3.3-3.4) associado às imagens Phantom 256 e Camera Man 256 variando o número de amostras $m \in\{2 k, 3 k, 4 k, 5 k\}$ e o nível de ruido $\sigma=\sigma_{x}=\sigma_{y} \in\{0.01,0.02, \ldots, 0.09\}$.

$\operatorname{PSNR}(\cdot, \cdot)$ associado à imagem recuperada fica por volta de $28 \mathrm{~dB}$ e, para o mesmo valor de $m$, 
quando o nível de ruido aumenta o valor do $P S N R$ desce até $22 \mathrm{~dB}$. O mesmo comportamento do $\operatorname{PSNR}(\cdot, \cdot)$ é observado para outros valores de $m$ sendo que o intervalo de variação do $\operatorname{PSNR}(\cdot, \cdot)$ é proporcional a m. O mesmo comportamento é observado na Figura 4.15.

As Figuras 4.16a-4.161 representam as imagens recuperadas nos problemas associados à imagem Phantom $256 \mathrm{com}$ nível de ruido $\sigma_{x}=\sigma_{y}=0.01$ e $m \in\{2 k, 3 k, 4 k, 5 k\}$. Na figura, a primeira coluna corresponde sempre à imagem com ruido, a segunda coluna corresponde à imagem utilizada como estimativa inicial do processo de otimização (quer dizer, a imagem vinda de considerar o coeficiente $s$ solução do problema (4.5)) e a terceira coluna corresponde à solução $x(\gamma)$ do problema (3.3-3.4) com maior valor de $\operatorname{PSNR}\left(x(\gamma), x^{*}\right)$. A primeira linha corresponde a $m=2 k$, a segunda corresponde a $m=3 k$ e a terceira e a quarta linha correspondem a $m=4 k$ e $m=5 k$, respectivamente. A Figura 4.16c, que corresponde à solução do problema com $\gamma=0.0104$ tem valor de $\operatorname{PSNR}\left(x(\gamma), x^{*}\right)=18.4054 \mathrm{~dB}$. Quando $m \geq 3$ a qualidade da imagem recuperada aumenta atingindo $42 \mathrm{~dB}$, no caso $m=5 k$.

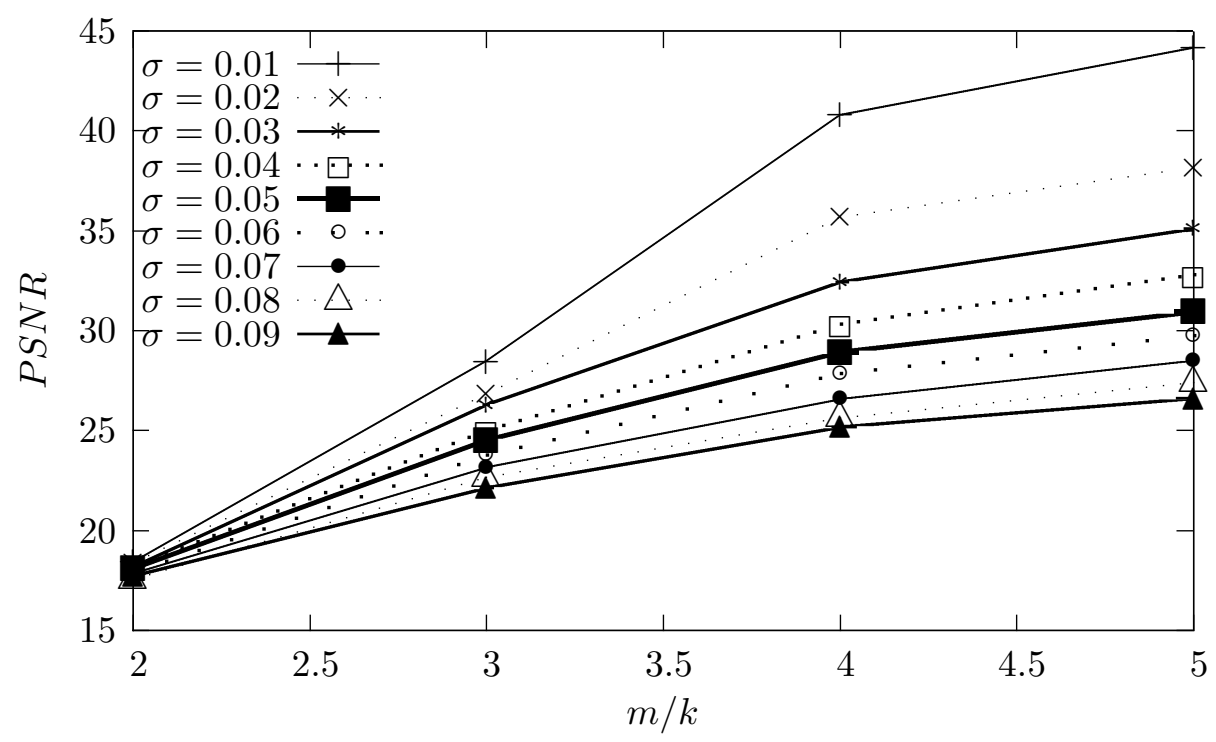

Figura 4.14: Comparação da qualidade das imagens recuperadas ao resolver os 36 problemas do tipo (3.3-3.4) associados à imagem Phantom 256 considerando diferentes níveis de ruido $\sigma_{x}=\sigma_{y} e$ diferentes números de amostras $m$.

Considerando o problema com $m=2 k$, a Figura 4.17 mostra a curva Pareto para $\gamma \in[0.9999,0.0001]$. Na figura, $f_{1}=\sum_{i=1}^{n} u_{i}+\sum_{i=1}^{n} v_{i}$ e $f_{2}=\|\Theta(u-v)-y\|_{2}^{2}$. A figura mostra ainda as imagens recuperadas correspondentes a alguns valores arbitrários de $\gamma$. Notamos que no intervalo $\gamma \in[0.2,0.0]$ as imagens recuperadas são mais parecidas à imagem original. Daqui que nos nossos experimentos consideramos $\gamma$ no intervalo $\left[\gamma_{\max }, \gamma_{\min }\right]$ com $\gamma_{\max }=0.2$ e $\gamma_{\min }=10^{-4}$.

As Figuras 4.18a-4.181 representam as imagens recuperadas nos problemas associados à imagem Camera Man 256 com nível de ruido $\sigma_{x}=\sigma_{y}=0.01$ e $m \in\{2 k, 3 k, 4 k, 5 k\}$. Na figura, a primeira coluna corresponde sempre à imagem com ruido, a segunda coluna corresponde à imagem utilizada como estimativa inicial do processo de otimização (quer dizer, a imagem vinda de considerar o coeficiente $s$ solução do problema (4.5)) e a terceira coluna corresponde à solução $x(\gamma)$ 


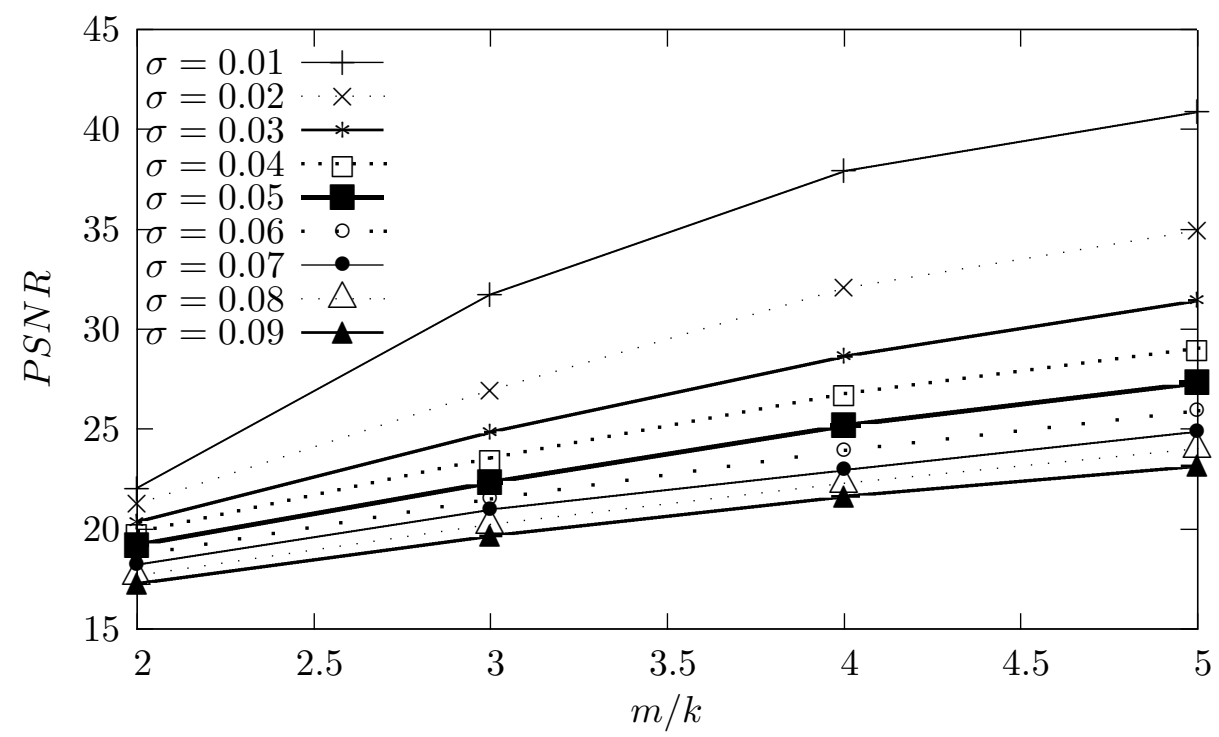

Figura 4.15: Comparação da qualidade das imagens recuperadas ao resolver os 36 problemas do tipo (3.3-3.4) associados à imagem Camera Man 256 considerando diferentes níveis de ruido $\sigma_{x}=\sigma_{y} e$ diferentes números de amostras $m$.

do problema (3.3-3.4) com maior valor de $\operatorname{PSNR}\left(x(\gamma), x^{*}\right)$. A primeira linha corresponde a $m=2 k$, a segunda corresponde a $m=3 k$ e a terceira e a quarta linea correspondem a $m=4 k$ e $m=5 k$, respectivamente. Figura 4.16c, que corresponde à solução do problema com $\gamma=0.0104$ tem valor de $\operatorname{PSNR}\left(x(\gamma), x^{*}\right)=18.4054 \mathrm{~dB}$. Quando $m \geq 3$ a qualidade da imagem recuperada aumenta atingindo $42 \mathrm{~dB}$, no caso $m=5 k$. A Figura $4.18 \mathrm{c}$, que corresponde à solução do problema com $\gamma=0.0073$ tem valor de $\operatorname{PSNR}\left(x(\gamma), x^{*}\right)=22.0350 \mathrm{~dB}$. Quando $m \geq 3$ a qualidade da imagem recuperada aumenta atingindo $38 \mathrm{~dB}$, no caso $m=5 k$.

Em muitos trabalhos que envolvem aplicações de compressive sensing à reconstrução de imagens, consideram-se imagens de teste não necessariamente esparsas na base $\Psi$. Neste contexto, em nossos experimentos consideraremos as seguintes imagens de $n=512 \times 512$ pixels: (a) Lena 512 , (b) Barbara 512 e (c) Mandrill 512. As três imagens, ilustradas na Figura 4.19, estão amplamente disponíveis na web (veja, por exemplo, [58]). A densidade das imagens Lena 512, Barbara 512 e Mandrill 512 é $94.35 \%, 95.89 \%$ e $97.92 \%$, respectivamente.

Nestes experimentos consideramos níveis de ruido $\sigma_{x}=\sigma_{y} \in\{0.01,0.02, \ldots, 0.05\}$ e um número de amostras dado por $m=\alpha n$ com $\alpha \in\{0.1,0.2, \ldots, 0.9\}$, obtendo um total de 135 problemas. A Tabela 4.8 mostra a qualidade das soluções, medida utilizando o $\operatorname{PSNR}(\cdot, \cdot)$, e o custo computacional de resolver cada um dos 135 problemas, apresentando o total de iterações (\# it), o total de avaliações de função (\# fcnt) e o tempo de CPU em segundos (Tempo) utilizados pelo SPG para resolver a sequência de 2,000 problemas variando $\gamma$ (para $\alpha$ e $\sigma$ fixos). Na tabela, $\alpha$ é tal que o número de amostras é dado por $m=\alpha n$ e $\sigma$ corresponde ao valor do nível de ruido $\sigma_{x}=\sigma_{y}=\sigma$. As Figuras 4.21-4.23 mostram a variação do $\operatorname{PSNR}(\cdot, \cdot)$ em função do nível de ruido e o número de amostras, para cada uma das imagens Lena 512, Barbara 512 e Mandrill 512, respectivamente. O comportamento é similar ao observado anteriormente. 


\begin{tabular}{|c|c|c|c|c|c|c|c|c|c|}
\hline \multirow{2}{*}{$m / k$} & \multirow[b]{2}{*}{$\sigma$} & \multicolumn{4}{|c|}{ Imagem Phantom 256} & \multicolumn{4}{|c|}{ Imagem Camera Man 256} \\
\hline & & $\operatorname{PSNR}(\cdot, \cdot)$ & \# it & \# fcnt & Tempo & $\operatorname{PSNR}(\cdot, \cdot)$ & \# it & \# fcnt & Tempo \\
\hline \multirow{9}{*}{2} & 0.1 & 18.409516 & 61,626 & 86,621 & 868.10 & 21.823839 & 43,262 & 67,429 & 538.93 \\
\hline & 0.2 & 27.984457 & 55,998 & 81,514 & 751.14 & 31.776533 & 38,082 & 63,576 & 494.99 \\
\hline & 0.3 & 40.856474 & 45,950 & 71,169 & 438.65 & 38.106914 & 29,698 & 55,306 & 336.62 \\
\hline & 0.4 & 44.136453 & 35,524 & 60,974 & 338.53 & 40.947919 & 27,582 & 53,167 & 357.76 \\
\hline & 0.5 & 18.213673 & 55,696 & 80,029 & 718.64 & 21.307818 & 38,354 & 61,732 & 514.47 \\
\hline & 0.6 & 26.983246 & 54,527 & 79,870 & 530.44 & 27.166329 & 35,214 & 59,985 & 400.27 \\
\hline & 0.7 & 35.717949 & 40,008 & 65,056 & 389.17 & 32.108234 & 30,702 & 55,823 & 341.98 \\
\hline & 0.8 & 38.368857 & 33,449 & 58,694 & 324.45 & 34.813227 & 26,676 & 51,658 & 448.58 \\
\hline & 0.9 & 18.014482 & 48,026 & 71,637 & 560.20 & 20.427230 & 36,121 & 58,815 & 578.75 \\
\hline \multirow{9}{*}{3} & 0.1 & 26.005193 & 54,298 & 79,386 & 565.41 & 24.982977 & 31,698 & 55,392 & 329.14 \\
\hline & 0.2 & 32.670810 & 43,189 & 68,071 & 410.88 & 28.745266 & 28,867 & 53,301 & 325.76 \\
\hline & 0.3 & 35.043807 & 35,005 & 60,033 & 354.22 & 31.433681 & 24,917 & 49,195 & 590.15 \\
\hline & 0.4 & 18.024570 & 53,838 & 77,889 & 616.29 & 19.767069 & 31,848 & 52,987 & 509.20 \\
\hline & 0.5 & 25.098360 & 49,844 & 74,700 & 471.93 & 23.382151 & 30,082 & 52,998 & 291.96 \\
\hline & 0.6 & 30.346729 & 42,122 & 66,810 & 404.90 & 26.664032 & 27,605 & 51,378 & 314.20 \\
\hline & 0.7 & 32.683462 & 33,057 & 57,796 & 336.87 & 29.040410 & 25,306 & 48,799 & 589.66 \\
\hline & 0.8 & 18.066545 & 51,699 & 75,638 & 594.36 & 19.300316 & 30,480 & 50,537 & 490.43 \\
\hline & 0.9 & 24.413126 & 48,331 & 72,705 & 417.23 & 22.383831 & 28,655 & 50,350 & 288.78 \\
\hline \multirow{9}{*}{4} & 0.1 & 28.922394 & 39,661 & 64,077 & 383.16 & 25.238877 & 25,822 & 48,396 & 293.87 \\
\hline & 0.2 & 30.756660 & 33,444 & 57,941 & 335.58 & 27.313324 & 22,125 & 44,669 & 536.62 \\
\hline & 0.3 & 18.097176 & 54,954 & 78,818 & 629.89 & 18.669803 & 26,560 & 45,290 & 438.62 \\
\hline & 0.4 & 23.681739 & 44,985 & 69,141 & 410.05 & 21.567593 & 25,824 & 46,095 & 265.72 \\
\hline & 0.5 & 27.725628 & 42,276 & 66,461 & 402.94 & 23.882798 & 23,559 & 44,970 & 270.51 \\
\hline & 0.6 & 29.594163 & 33,652 & 57,927 & 673.76 & 26.014848 & 21,049 & 42,526 & 508.13 \\
\hline & 0.7 & 17.708898 & 50,346 & 74,073 & 533.62 & 18.224832 & 23,978 & 41,013 & 325.79 \\
\hline & 0.8 & 22.857610 & 44,944 & 68,897 & 408.24 & 20.857925 & 23,762 & 43,237 & 246.77 \\
\hline & 0.9 & 26.491847 & 38,030 & 62,086 & 371.31 & 22.949558 & 21,916 & 41,668 & 243.16 \\
\hline \multirow{9}{*}{5} & 0.1 & 28.447949 & 31,171 & 55,017 & 714.10 & 24.855008 & 20,990 & 41,183 & 399.04 \\
\hline & 0.2 & 17.746482 & 49,347 & 72,233 & 533.00 & 17.794731 & 22,207 & 37,649 & 351.19 \\
\hline & 0.3 & 22.279440 & 45,757 & 69,839 & 413.95 & 20.145172 & 20,787 & 38,502 & 215.48 \\
\hline & 0.4 & 25.846373 & 37,047 & 60,749 & 361.54 & 22.307714 & 19,771 & 38,405 & 209.30 \\
\hline & 0.5 & 27.321747 & 34,711 & 58,366 & 772.67 & 23.998037 & 19,722 & 38,684 & 355.61 \\
\hline & 0.6 & 17.486831 & 46,910 & 69,636 & 607.07 & 17.355597 & 18,807 & 33,183 & 264.81 \\
\hline & 0.7 & 21.887356 & 44,879 & 68,681 & 421.23 & 19.681704 & 19,142 & 35,525 & 198.54 \\
\hline & 0.8 & 24.931380 & 35,675 & 58,984 & 347.10 & 21.435238 & 17,955 & 35,107 & 217.98 \\
\hline & 0.9 & 26.612839 & 29,883 & 52,957 & 686.60 & 23.163715 & 18,570 & 36,391 & 336.58 \\
\hline
\end{tabular}

Tabela 4.7: Detalhe da qualidade das soluções e do desempenho do SPG na resolução do problema (3.3-3.4) para as imagens Phantom 256 e Camera Man 256 variando o nível de ruido $\sigma_{x}=\sigma_{y} e$ o número de amostras $m$.

As Figuras 4.20a-i, 4.20j-r e 4.20s-aa mostram uma comparação das curvas Pareto para valores fixos do número de amostras e variando o nível de ruido nos experimentos com as imagens Lena 512, Barbara 512 e Mandrill 512, respectivamente. As Figuras 4.14 e 4.15 mostram o valor de $\operatorname{PSNR}\left(x, x^{*}\right)$ para as 36 combinações de nível de ruido e número de amostras nos experimentos envolvendo as imagens Phantom 256 e Camera Man 256, respectivamente. Para cada combinação de $\sigma_{x}=\sigma_{y}$ e $m$, como já foi mencionado anteriormente, consideramos como imagem recuperada $x$, dentre todas as 2,000x $x(\gamma)$, aquela com maior valor de $\operatorname{PSNR}\left(x(\gamma), x^{*}\right)$. As Figuras 4.24-4.26 mostram as imagens recuperadas considerando nível de ruido $\sigma_{x}=\sigma_{y}=$ 0.01 para diferentes valores do número de amostras $m=\alpha n$ com $\alpha \in\{0.1,0.2, \ldots, 0.9\}$, para as imagens Lena 512, Barbara 512 e Mandrill 512, respectivamente.

Analisando a Figura 4.24, notamos que para $m \geq 0.4 n$ as imagens resultantes são muito parecidas à imagem original, com valor de $\operatorname{PSNR}\left(x(\gamma), x^{*}\right)=23 \mathrm{~dB}$ no caso $m=0.4 n$. A medida que aumenta o número de amostras a qualidade das imagens recuperadas melhora até atingir um valor de $\operatorname{PSNR}\left(x(\gamma), x^{*}\right)=40.6208 \mathrm{~dB}$ no caso $m=0.9 n$. Analisando a Figura 4.25 , notamos 


\begin{tabular}{|c|c|c|c|c|c|c|c|c|c|c|c|c|c|}
\hline & & \multicolumn{4}{|c|}{ Imagem Lena 512} & \multicolumn{4}{|c|}{ Imagem Barbara 512} & \multicolumn{4}{|c|}{ Imagem Mandrill 512} \\
\hline$\alpha$ & $\sigma$ & $\operatorname{PSNR}(\cdot, \cdot)$ & \# it & \# fcnt & Tempo & $\operatorname{PSNR}(\cdot, \cdot)$ & \# it & \# fcnt & Tempo & $\operatorname{PSNR}(\cdot, \cdot)$ & \# it & \# fcnt & Tempo \\
\hline \multirow{5}{*}{0.1} & 0.01 & 16.134409 & 82,802 & 110,287 & $3,049.40$ & 15.123786 & 88,389 & 116,016 & $3,413.62$ & 16.896500 & 76,678 & 104,688 & $2,886.91$ \\
\hline & 0.02 & 19.182680 & 75,697 & 102,595 & $3,637.82$ & 17.115679 & 87,288 & 114,714 & $4,107.47$ & 18.140199 & 80,474 & 107,624 & $3,730.33$ \\
\hline & 0.03 & 22.223677 & 65,998 & 92,327 & $3,607.19$ & 19.043394 & 79,314 & 106,163 & $3,682.90$ & 19.418215 & 75,554 & 102,379 & $3,044.71$ \\
\hline & 0.04 & 25.147852 & 55,928 & 82,015 & $2,376.53$ & 20.895304 & 73,597 & 100,379 & $4,115.48$ & 20.839047 & 70,839 & 97,607 & $2,905.56$ \\
\hline & 0.05 & 28.230556 & 48,435 & 75,067 & $2,269.22$ & 22.831951 & 63,147 & 90,535 & $2,744.33$ & 22.443594 & 64,378 & 91,755 & $2,648.26$ \\
\hline \multirow{5}{*}{0.2} & 0.01 & 31.380095 & 43,053 & 70,008 & $3,109.59$ & 25.011818 & 59,885 & 87,436 & $3,442.34$ & 24.416165 & 62,225 & 90,000 & $2,909.63$ \\
\hline & 0.02 & 34.485758 & 38,204 & 65,162 & $2,134.58$ & 27.698817 & 55,258 & 82,653 & $2,826.47$ & 26.955540 & 55,064 & 82,792 & $2,715.32$ \\
\hline & 0.03 & 37.219963 & 36,149 & 63,374 & $2,399.03$ & 30.620268 & 50,297 & 77,834 & $2,861.75$ & 30.718425 & 49,892 & 77,776 & $2,753.63$ \\
\hline & 0.04 & 39.256680 & 33,220 & 60,488 & $2,050.83$ & 33.808677 & 44,806 & 72,356 & $2,520.65$ & 35.199277 & 42,385 & 70,216 & $2,373.19$ \\
\hline & 0.05 & 15.988598 & 84,238 & 111,875 & $3,029.58$ & 15.050359 & 91,597 & 119,489 & $3,985.91$ & 16.804260 & 80,546 & 108,505 & $2,952.01$ \\
\hline \multirow{5}{*}{0.3} & 0.01 & 18.842025 & 77,386 & 104,423 & $3,657.35$ & 16.986453 & 90,474 & 118,026 & $4,254.91$ & 18.030635 & 82,606 & 109,693 & $3,631.06$ \\
\hline & 0.02 & 21.657692 & 66,577 & 92,887 & 3,500 & 18.855817 & 80,341 & 107,233 & 4,1 & 19.254354 & 78,851 & 105,711 & $3,060.96$ \\
\hline & 0.03 & 24.168987 & 60,827 & 87,112 & $3,091.16$ & 20.624002 & 74,941 & 101,804 & $4,267.90$ & 20.600246 & 74,681 & 101,491 & $3,132.34$ \\
\hline & 0.04 & 26.583914 & 51,229 & 78,044 & $2,656.79$ & 22.425985 & 65,326 & 92,674 & $2,630.27$ & 22.055220 & 64,921 & 92,400 & $2,675.91$ \\
\hline & 0.05 & 28.730495 & 48,099 & 75,274 & $3,300.80$ & 24.320550 & 62,046 & 89,700 & $3,478.73$ & 23.761298 & 63,193 & 91,035 & $2,914.97$ \\
\hline \multirow{5}{*}{0.4} & 0.01 & 30.612428 & 45,246 & 72,544 & $2,637.61$ & 26.649789 & 59,175 & 86,681 & $3,353.12$ & 25.678865 & 59,506 & 87,442 & 2,88 \\
\hline & 0.02 & 32.312896 & 41,969 & 69,461 & $2,684.73$ & 28.792889 & 54,820 & 82,453 & $3,927.67$ & 27.904890 & 55,454 & 83,671 & $2,905.15$ \\
\hline & 0.03 & 33.796002 & 39,720 & 67,384 & $2,233.01$ & 30.963152 & 49,242 & 76,922 & $2,959.18$ & 30.231985 & 49,136 & 77,378 & $2,677.91$ \\
\hline & 0.04 & 15.800674 & 91,227 & 118,924 & $3,166.60$ & 14.943682 & 95,644 & 123,569 & 3,9 & 16.685004 & 84,741 & 112,799 & $3,938.03$ \\
\hline & 0.05 & 18.424374 & 82,884 & 110,136 & 3,89 & 16.794196 & 94,090 & 121,820 & 4,67 & 17.862762 & 84,918 & 112,140 & $3,451.80$ \\
\hline \multirow{5}{*}{0.5} & 0.01 & 21.002748 & 71,793 & 98,406 & $3,611.62$ & 18.590492 & 84,472 & 111,494 & $4,415.35$ & 19.023686 & 81,424 & 108,428 & $3,218.39$ \\
\hline & 0.02 & 23.192096 & 65,758 & 92,157 & $3,441.34$ & 20.258638 & 79,543 & 106,529 & $4,830.51$ & 20.279064 & 76,742 & 103,679 & $3,243.59$ \\
\hline & 0.03 & 25.227093 & 57,295 & 84,267 & 3,29 & 21.910707 & 66,828 & 94,339 & 2,6 & 21.574156 & 68,676 & 96,251 & $2,803.59$ \\
\hline & 0.04 & 26.965989 & 3,135 & 80,582 & 2,8 & 35201 & 64,560 & 92,329 & & 38004 & 68,990 & 97,132 & 3,0 \\
\hline & 0.05 & 28.436493 & 51,582 & 79,219 & $3,057.27$ & 25.613623 & 62,114 & 89,687 & 3,9 & 51021 & 65,054 & 93,280 & $3,096.27$ \\
\hline \multirow{5}{*}{0.6} & 0.01 & 29.769490 & 48,853 & 76,629 & $3,067.12$ & 27.354663 & 59,502 & 87,168 & 3,49 & 26.174275 & 60,389 & 88,789 & $3,043.70$ \\
\hline & 0.02 & 30.958355 & 45,359 & 73,421 & $2,683.42$ & 29.054909 & 54,405 & 82,170 & 3,65 & 27.806249 & 55,635 & 84,212 & $2,957.18$ \\
\hline & 0.03 & 15.599433 & 94,701 & 122,791 & 3,39 & 14.826419 & 99,301 & 127,433 & 4,4 & 16.553692 & 90,156 & 118,375 & 3,86 \\
\hline & 0.04 & 17.990314 & 89,942 & 117,446 & $4,191.58$ & 16.573480 & 96,727 & 124,580 & 4,71 & 17.669059 & 88,595 & 115,943 & $3,648.66$ \\
\hline & 0.05 & 20.356678 & 75,658 & 102,463 & $4,078.45$ & 18.291407 & 86,756 & 113,872 & $4,418.23$ & 18.759032 & 84,252 & 111,397 & $3,264.74$ \\
\hline \multirow{5}{*}{0.7} & 0.01 & 22.325571 & 69,225 & 95,851 & $3,726.10$ & 19.858993 & 81,351 & 108,420 & $4,829.41$ & 19.926116 & 81,223 & 108,171 & $3,346.56$ \\
\hline & 0.02 & 24.109623 & 61,698 & 88,828 & $3,648.85$ & 21.379813 & 72,431 & 100,227 & $3,421.75$ & 21.078890 & 72,537 & 100,240 & $3,148.48$ \\
\hline & 0.03 & 25.643452 & 58,869 & 86,459 & $2,912.42$ & 23.134685 & 68,198 & 95,753 & $3,666.02$ & 22.356278 & 71,217 & 99,363 & $3,177.05$ \\
\hline & 0.04 & 26.901867 & 57,410 & 85,400 & $3,345.80$ & 24.707792 & 65,906 & 93,546 & $3,751.88$ & 23.618461 & 68,593 & 96,909 & $3,410.31$ \\
\hline & 0.05 & 28.042120 & 53,678 & 81,767 & $2,839.50$ & 26.202909 & 65,302 & 93,112 & $4,099.87$ & 24.912258 & 65,881 & 94,705 & $3,238.81$ \\
\hline \multirow{5}{*}{0.8} & 0.01 & 29.063366 & 54,605 & 83,277 & $3,572.60$ & 27.646264 & 60,986 & 88,802 & $3,342.33$ & 26.211162 & 61,684 & 90,674 & $3,130.12$ \\
\hline & 0.02 & 15.412279 & 99,720 & 127,874 & $3,598.71$ & 14.703542 & 103,577 & 131,923 & $4,814.37$ & 16.434951 & 98,647 & 126,896 & $3,931.39$ \\
\hline & 0.03 & 17.575804 & 91,922 & 119,639 & $4,519.51$ & 16.342378 & 99,485 & 127,525 & $3,720.71$ & 17.480346 & 94,160 & 121,768 & $3,451.02$ \\
\hline & 0.04 & 19.765489 & 83,367 & 110,542 & $3,235.32$ & 17.987199 & 92,439 & 119,812 & $4,107.90$ & 18.496999 & 88,671 & 116,000 & $3,675.66$ \\
\hline & 0.05 & 21.571499 & 74,410 & 101,112 & $4,168.58$ & 19.461096 & 84,687 & 111,977 & $4,402.72$ & 19.572596 & 85,792 & 112,985 & $3,446.37$ \\
\hline \multirow{5}{*}{0.9} & 0.01 & 23.182220 & 67,475 & 94,851 & $4,002.22$ & 20.863693 & 75,771 & 103,537 & $3,663.68$ & 20.612827 & 77,830 & 105,643 & $3,270.37$ \\
\hline & 0.02 & 24.577694 & 65,806 & 93,588 & $2,887.12$ & 22.529364 & 74,293 & 101,914 & $3,337.96$ & 21.745420 & 73,295 & 101,462 & $3,265.14$ \\
\hline & 0.03 & 25.715906 & 62,503 & 90,625 & $3,597.15$ & 23.930635 & 70,690 & 98,387 & $3,641.95$ & 22.837005 & 73,364 & 101,912 & $3,766.93$ \\
\hline & & 26.733406 & 62,219 & 90,848 & & 25.260248 & 69,730 & 97,567 & & 23.917336 & 71,837 & 100,948 & $3,414.29$ \\
\hline & 0.05 & 27.647914 & 59,257 & 88,195 & $3,371.13$ & 26.537841 & 66,887 & 94,843 & $3,431.79$ & 25.011217 & 68,846 & 98,299 & $3,515.24$ \\
\hline
\end{tabular}

Tabela 4.8: Detalhe da qualidade das soluções e do desempenho do SPG na resolução do problema (3.3-3.4) para as imagens Lena 512, Barbara 512 e Madrill 512 variando o nível de ruido $\sigma_{x}=\sigma_{y}$ e o número de amostras $m$.

que para $m \geq 0.5 n$ as imagens resultantes são muito parecidas à imagem original, com valor de $P S N R\left(x(\gamma), x^{*}\right)=22 \mathrm{~dB}$ no caso $m=0.5 n$. A medida que aumenta o número de amostras a qualidade das imagens recuperadas melhora até atingir um valor de $\operatorname{PSNR}\left(x(\gamma), x^{*}\right)=33.80867 \mathrm{~dB}$ no caso $m=0.9 n$. Finalmente, analisando a Figura 4.26 , notamos que para $m \geq 0.4 n$ as imagens resultantes são muito parecidas à imagem original, com valor de $\operatorname{PSNR}\left(x(\gamma), x^{*}\right)=20 \mathrm{~dB}$ no caso $m=0.4 n$. A medida que aumenta o número de amostras a qualidade das imagens recuperadas melhora até atingir um valor de $\operatorname{PSNR}\left(x(\gamma), x^{*}\right)=36.05327 \mathrm{~dB}$ no caso $m=0.9 n$.

\subsection{Experimentos de grande porte}

Nesta seção, para verificar o desempenho do SPG ao resolver o problema (3.3-3.4), realizamos experimentos numéricos com imagens com $n=1,024 \times 1,024=1,04 x x 8,576$ e $n=2,048 \times$ 
$2,048=4,194,304$ pixels. Imagens de $n=4,096 \times 4,096$ pixels não puderam ser testadas por falta de memória no ambiente computacional descrito no inicio do capítulo. Note que o número de variáveis dos problemas de otimização considerados é $2 n$, quer dizer, que resolveremos problemas de otimização com até $8,338,608$ de variáveis. A variação do parâmetro $\gamma$ é como segue

$$
\gamma \in\{0.2000,0.1995, \ldots, 0.0001\}
$$

Na Figura 4.28 ilustramos o teste para a imagem Phantom de 1, $024 \times 1,024$ pixels. A Figura 4.28c é a imagem recuperada com $m=5 k$ onde $k=36,956$, apresentando um total iterações de 18,375, um total de avaliações de função de 22,546 e um tempo de CPU em segundos (Tempo) utilizados pelo SPG para resolver a sequência de 400 problemas variando $\gamma$ (para $\sigma$ fixo) de 3,893.43.

Mostramos na Figura 4.28 o teste para a imagem Phantom de $n=2,048 \times 2,048$ pixels. A Figura 4.28c ilustra a imagem recuperada com $m=5 k$ onde $k=85,853$, apresentando um total iterações de 27,884, um total de avaliações de função de 32,332 e um tempo de CPU em segundos (Tempo) utilizados pelo SPG para resolver a sequência de 400 problemas variando $\gamma$ (para $\sigma$ fixo) de 25468.68. 


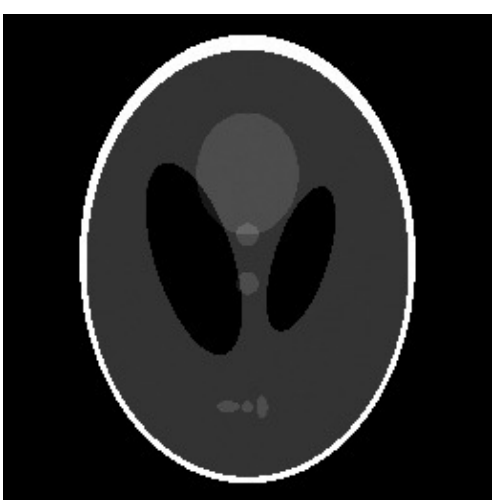

(a)

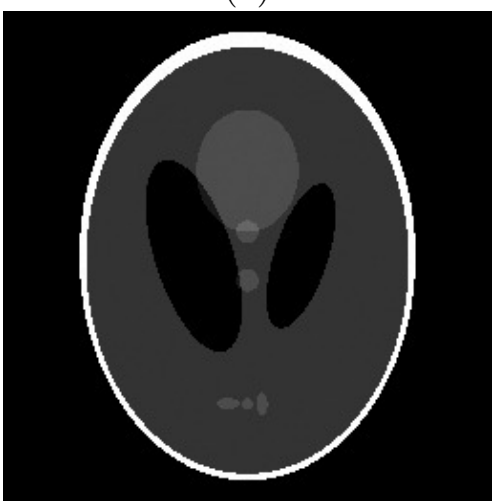

(d)

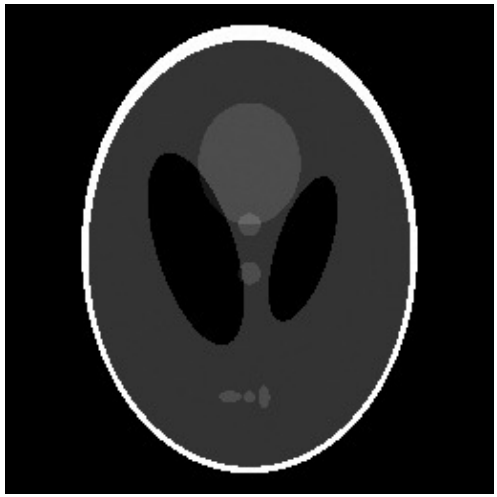

(g)

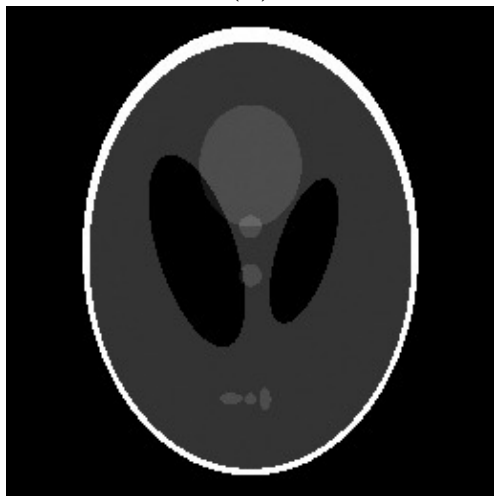

(j)

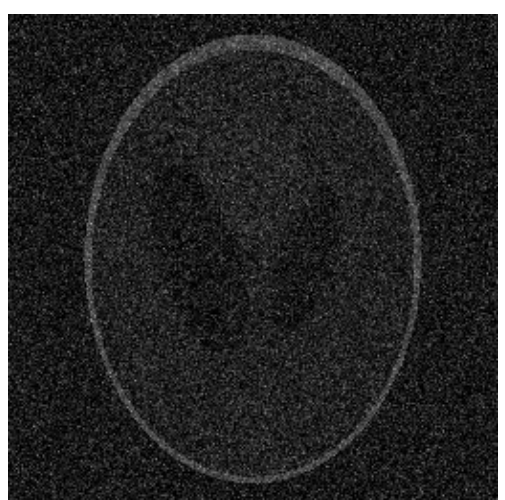

(b)

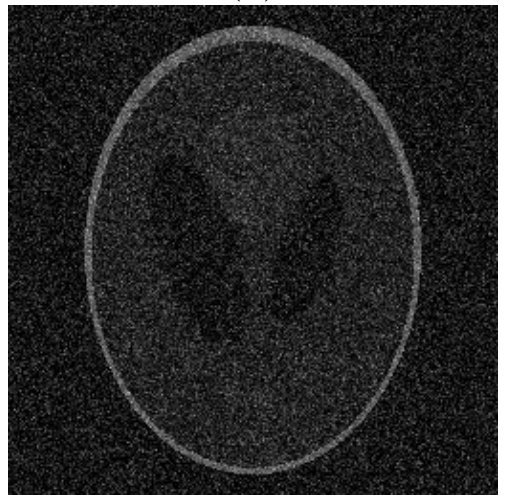

(e)

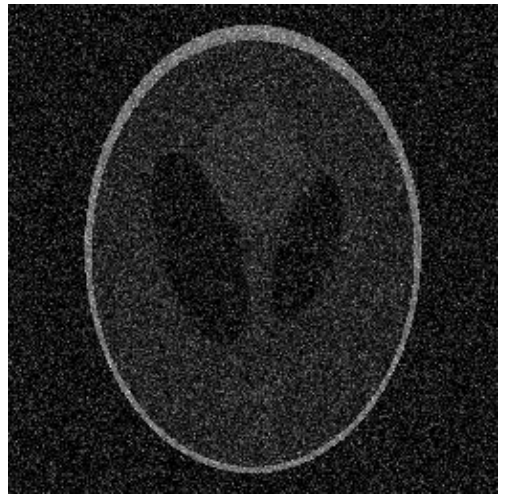

(h)

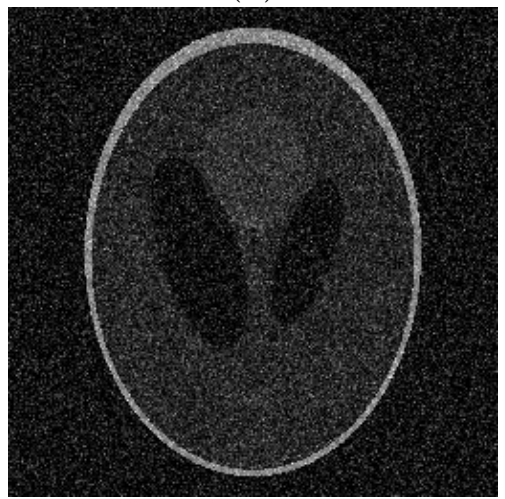

(k)

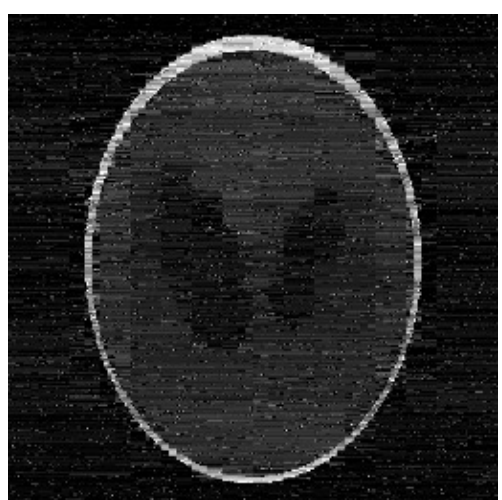

(c) $\gamma=0.0104$

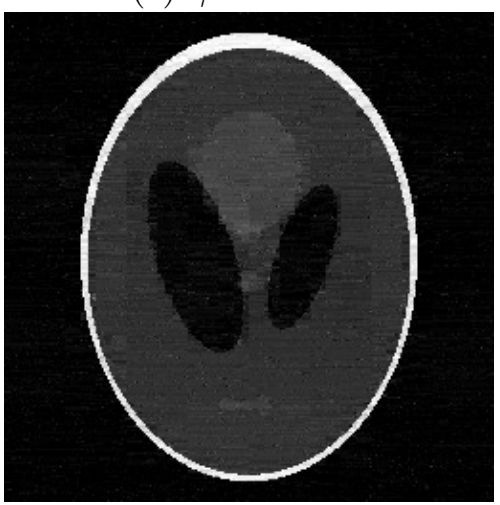

(f) $\gamma=0.0013$

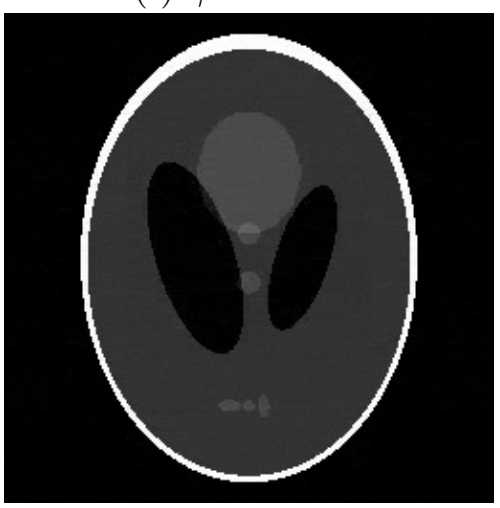

(i) $\gamma=0.0009$

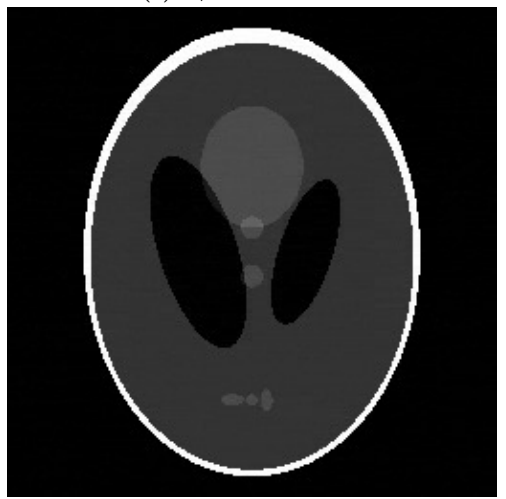

(l) $\gamma=0.0018$

Figura 4.16: Comparação da imagem original Phantom 256 com nível de ruido $\sigma_{x}=\sigma_{y}=0.001$ ((a) $m=2 k$, (d) $m=3 k$, (g) $m=4 k e(j) m=5 k$ ) com a imagem recuperada resolvendo o problema (4.5)) ((b) $m=2 k$, (e) $m=3 k$, (h) $m=4 k$ e (k) $m=5 k$ ) e a imagem recuperada resolvendo o problema (3.3-3.4) ((c) $m=2 k$, (f) $m=3 k$, (i) $m=4 k$ e (l) $m=5 k$ ), para $m \in\{2 k, 3 k, 4 k, 5 k\}$. 


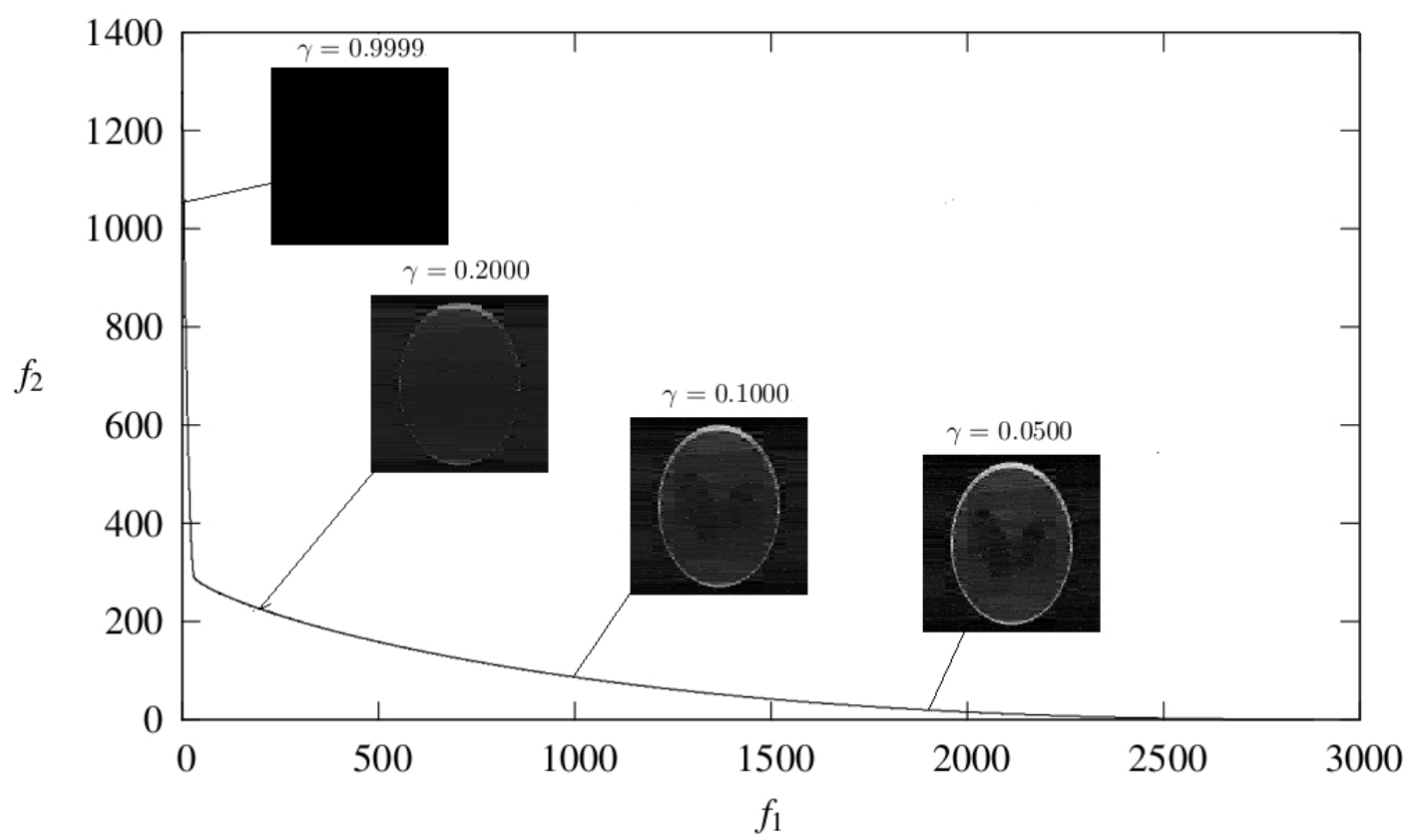

Figura 4.17: Curva Pareto do problema (3.3-3.4) associado à imagem Phantom 256 com nível de ruido $\sigma_{x}=\sigma_{y}=0.01$ e número de amostras $m=2 k$. 


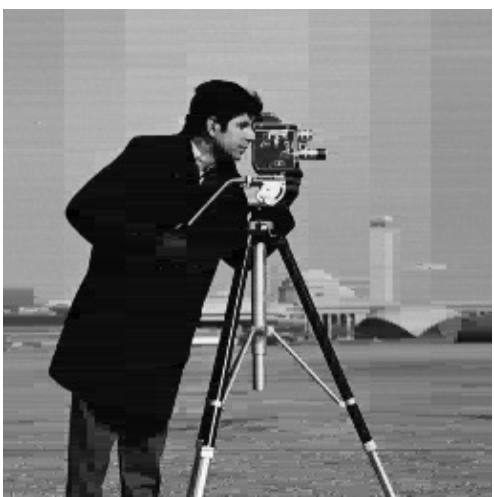

(a)

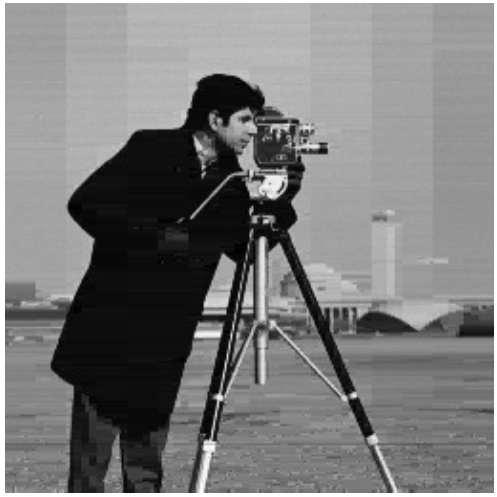

(d)

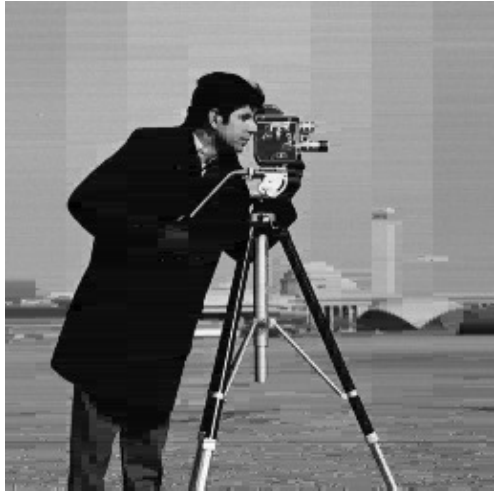

(g)

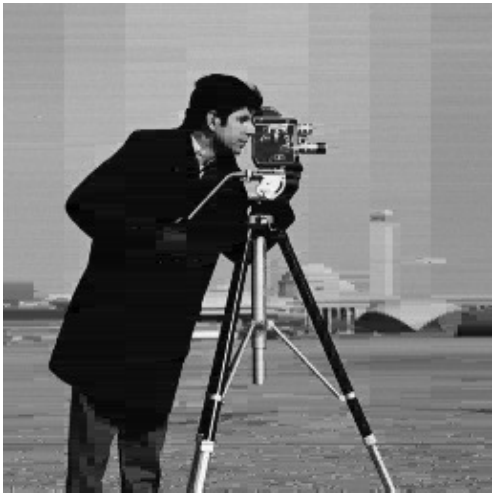

(j)

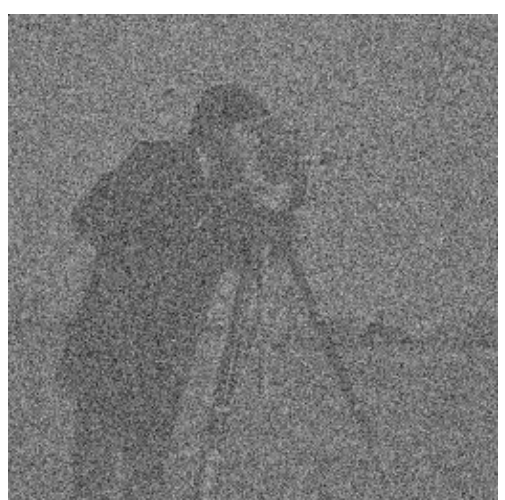

(b)

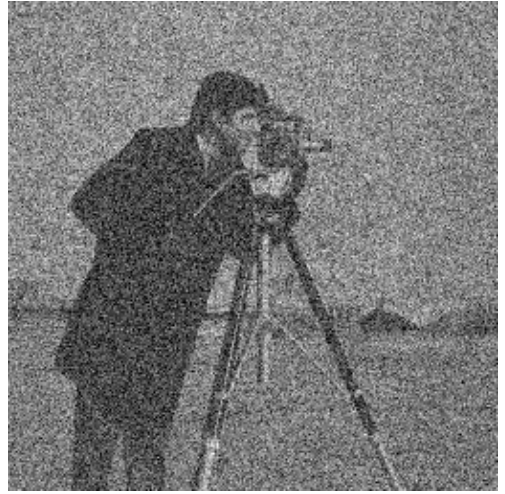

(e)

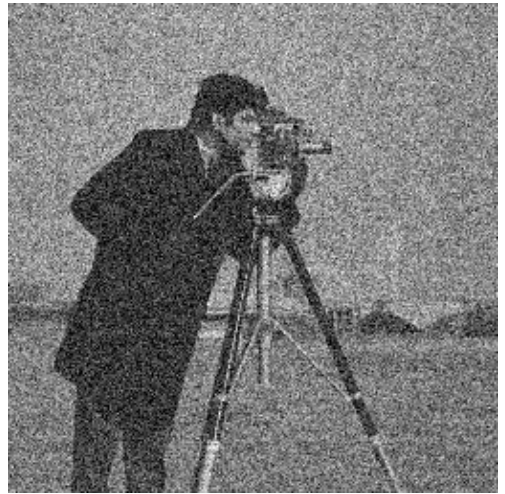

(h)

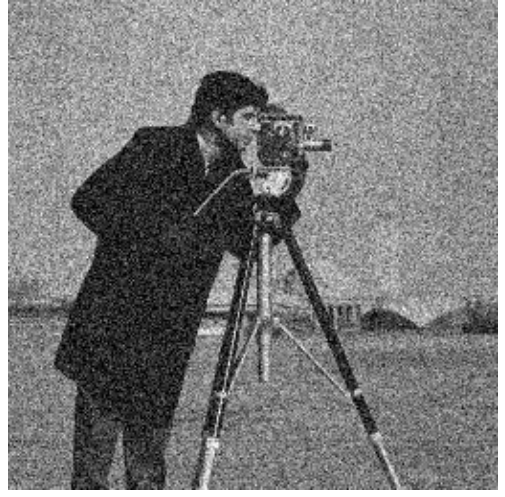

(k)

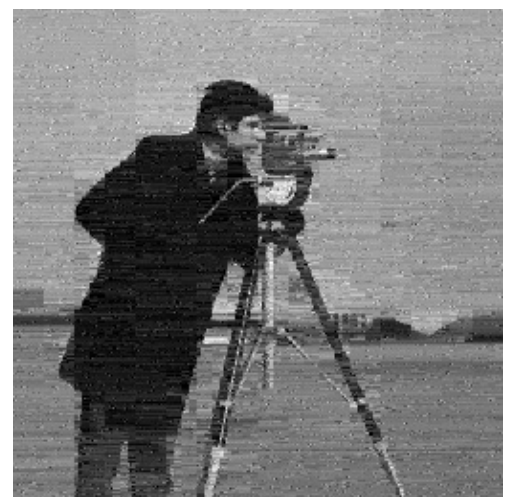

(c) $\gamma=0.0073$

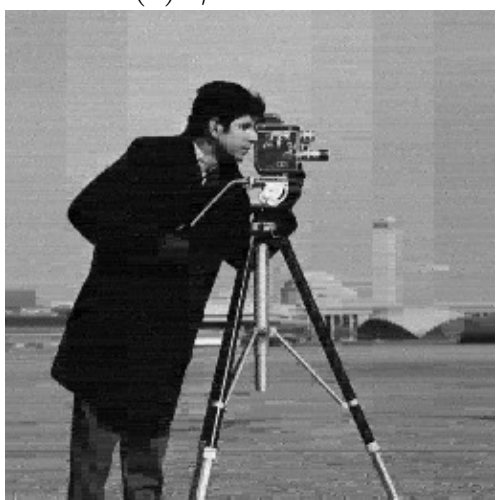

(f) $\gamma=0.003$

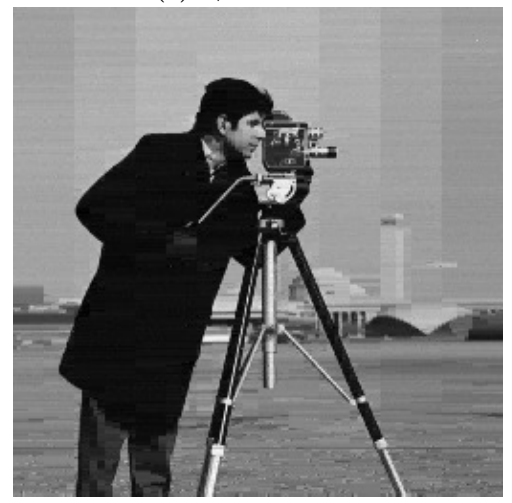

(i) $\gamma=0.0047$

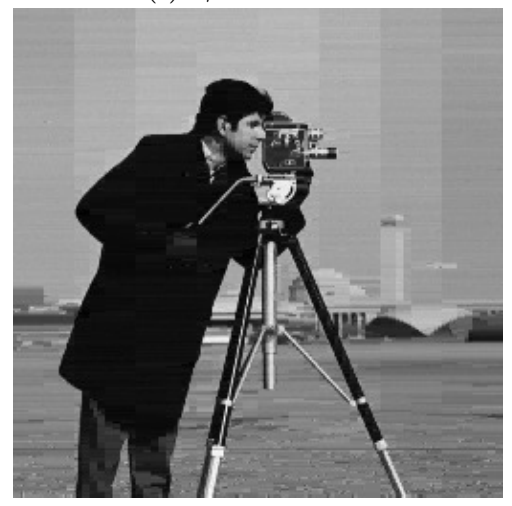

(l) $\gamma=0.0059$

Figura 4.18: Comparação da imagem original Camera Man 256 com nível de ruido $\sigma_{x}=\sigma_{y}=0.001$ ((a) $m=2 k$, (d) $m=3 k$, (g) $m=4 k e(j) m=5 k$ ) com a imagem recuperada resolvendo o problema (4.5)) ((b) $m=2 k$, (e) $m=3 k$, (h) $m=4 k$ e (k) $m=5 k$ ) e a imagem recuperada resolvendo o problema (3.3-3.4) ((c) $m=2 k$, (f) $m=3 k$, (i) $m=4 k$ e (l) $m=5 k$ ), para $m \in\{2 k, 3 k, 4 k, 5 k\}$. 


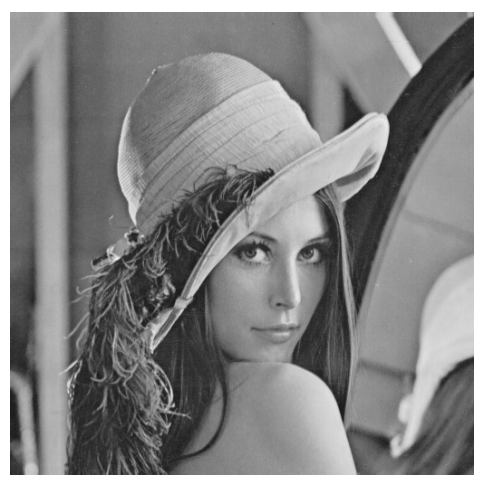

(a)

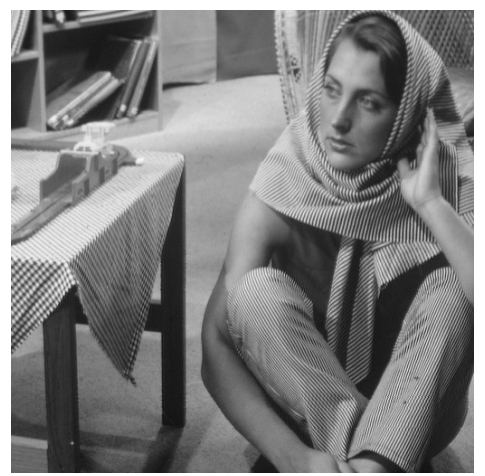

(b)

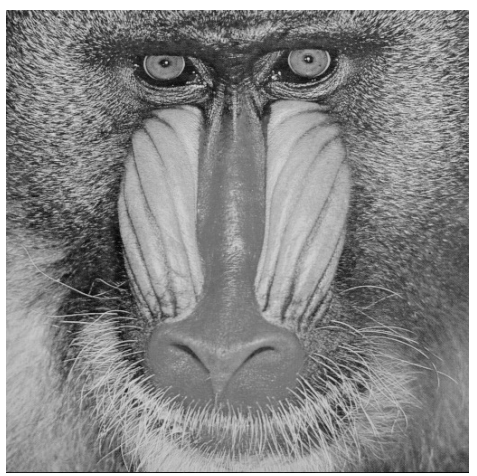

(c)

Figura 4.19: Imagens não esparsas (a) Lena, (b) Barbara e (c) Mandrill, todas com $n=512 \times 512$ pixels. 
Imagem Lena 512
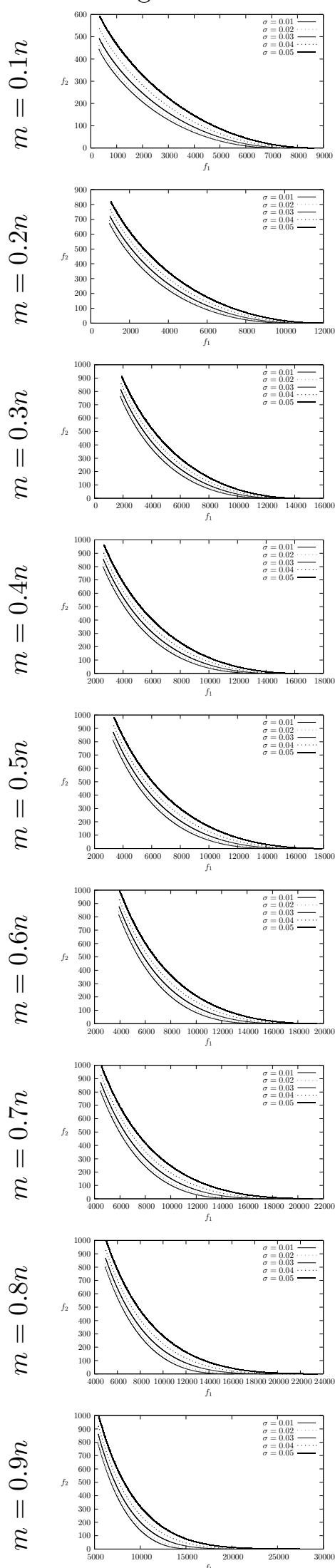

Imagem Barbara 512
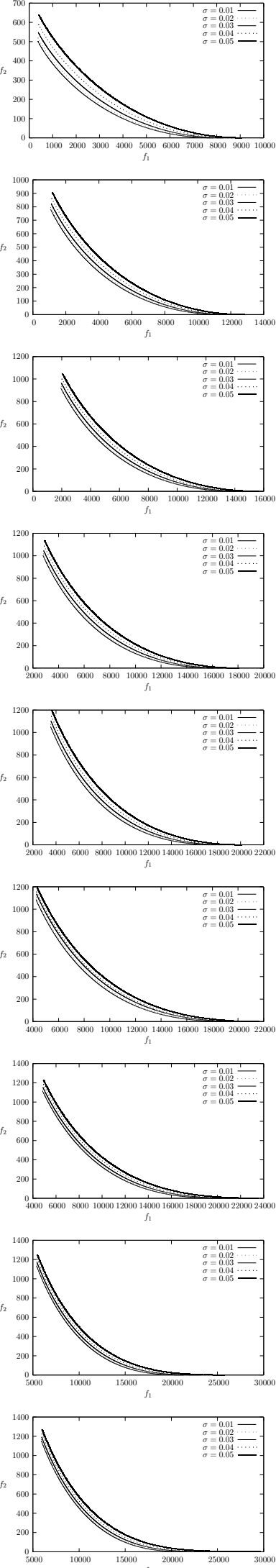

Imagem Mandrill 512
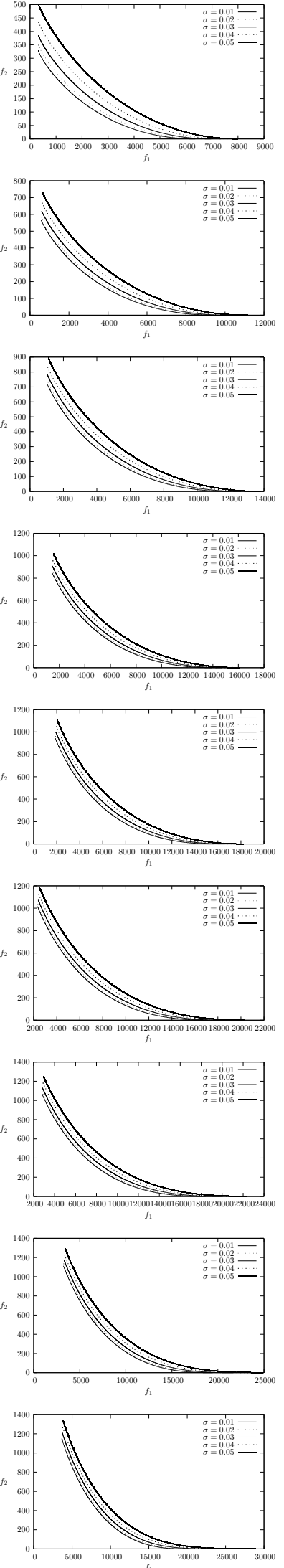

Figura 4.20: Comportamento das curvas Pareto do problema (3.3-3.4) associado às imagens Lena 512, Barbara 512 e Mandrill 512 variando o número de amostras $m \in\{0.1 n, 0.2 n, \ldots, 0.9 n\}$ e o nível de ruido $\sigma=\sigma_{x}=\sigma_{y} \in\{0.01,0.02, \ldots, 0.05\}$. 


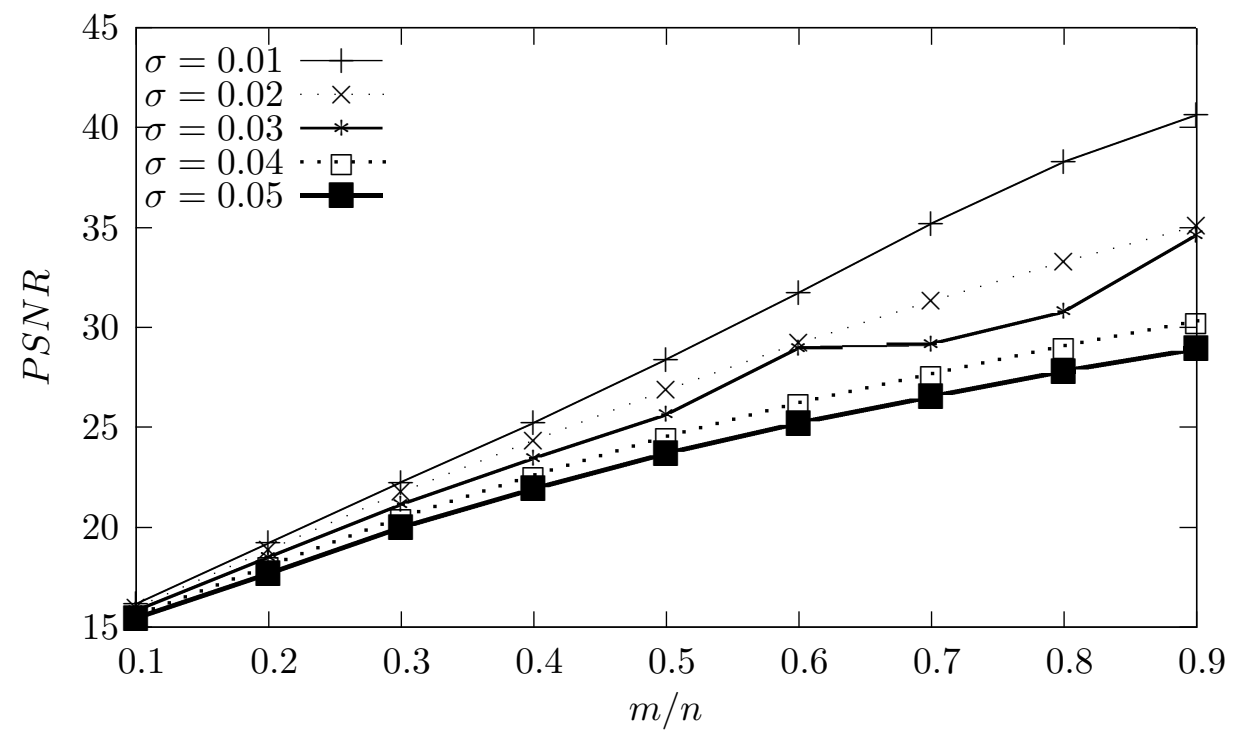

Figura 4.21: Comparação da qualidade das imagens recuperadas ao resolver os 45 problemas do tipo (3.3-3.4) associados à imagem Lena 512 considerando diferentes níveis de ruido $\sigma_{x}=\sigma_{y}$ e diferentes números de amostras $m$.

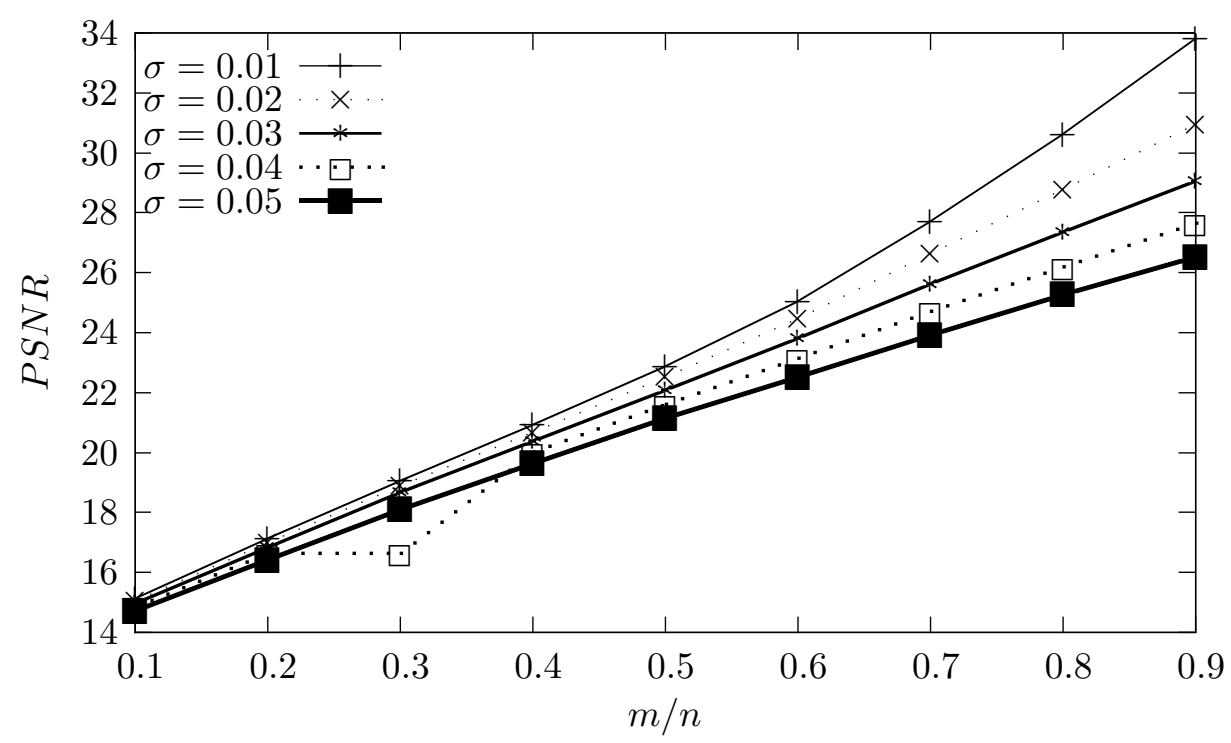

Figura 4.22: Comparação da qualidade das imagens recuperadas ao resolver os 45 problemas do tipo (3.3-3.4) associados à imagem Barbara 512 considerando diferentes níveis de ruido $\sigma_{x}=\sigma_{y} e$ diferentes números de amostras $m$. 


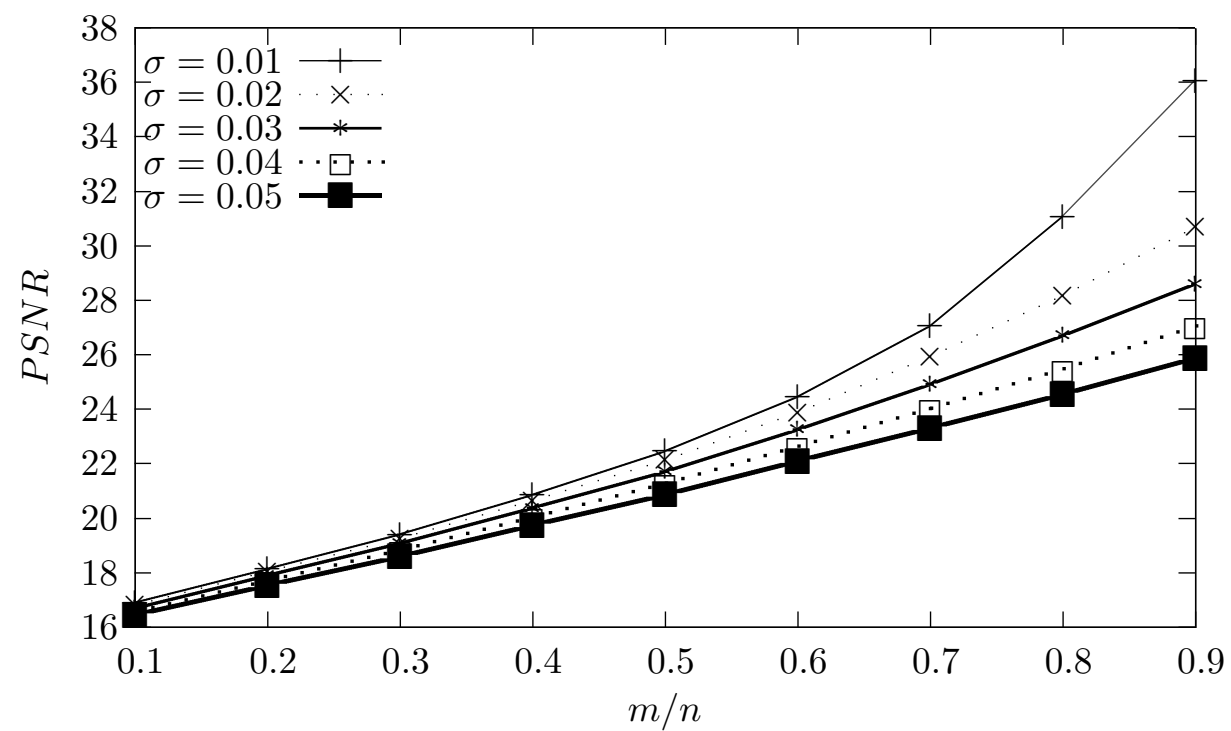

Figura 4.23: Comparação da qualidade das imagens recuperadas ao resolver os 45 problemas do tipo (3.3-3.4) associados à imagem Mandrill 512 considerando diferentes níveis de ruido $\sigma_{x}=\sigma_{y} e$ diferentes números de amostras $m$. 


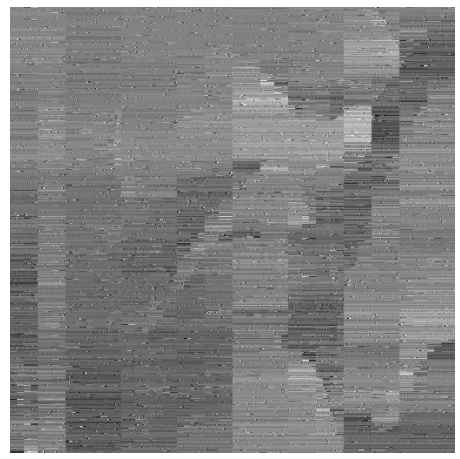

(a) $m=0.1 n$

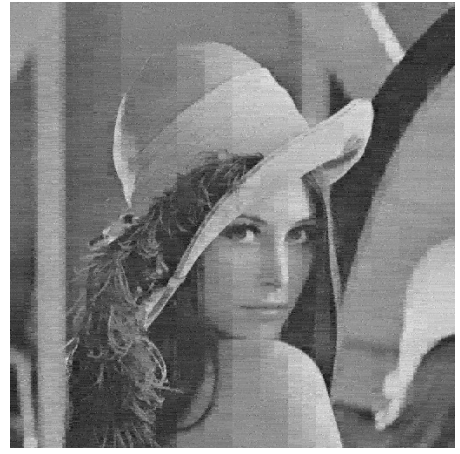

(d) $m=0.4 n$

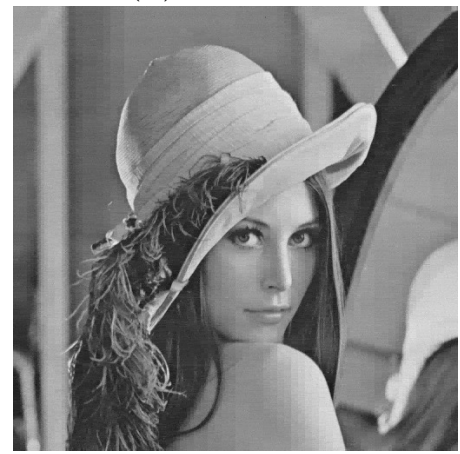

(h) $m=0.7 n$

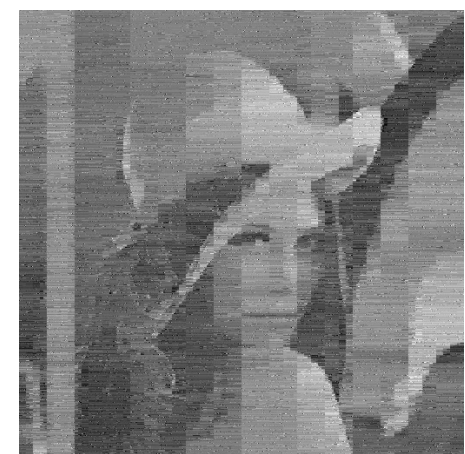

(b) $m=0.2 n$

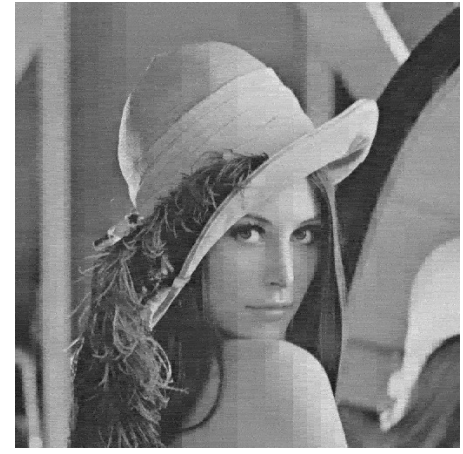

(e) $m=0.5 n$

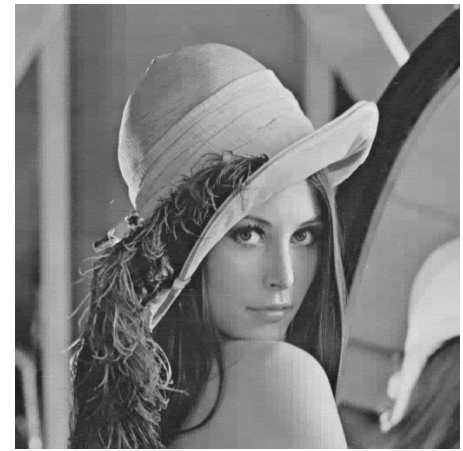

(i) $m=0.8 n$

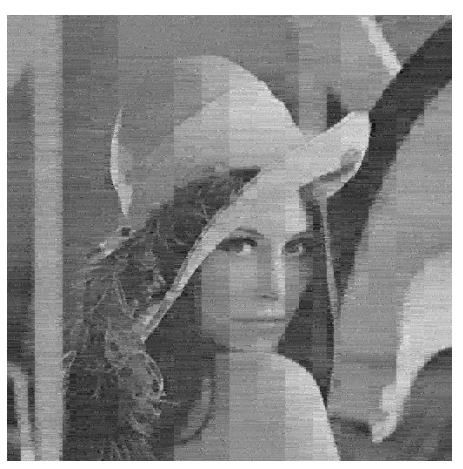

(c) $m=0.3 n$

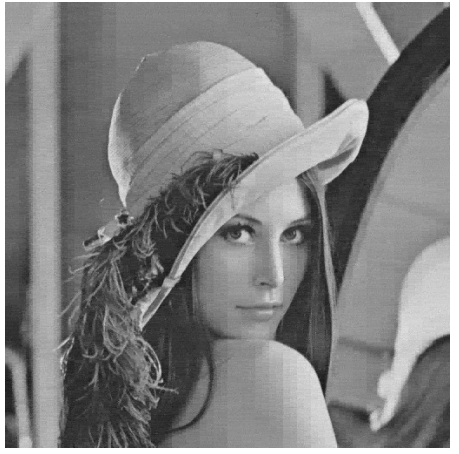

(g) $m=0.6 n$

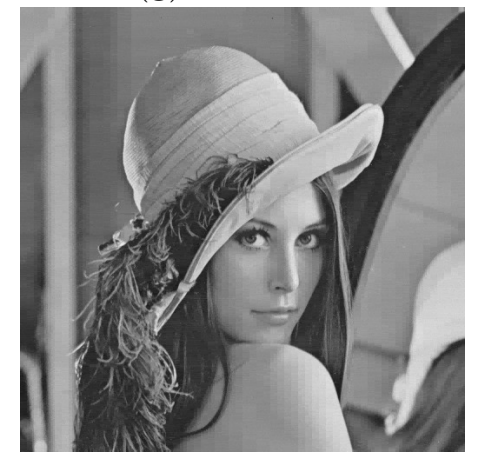

(j) $m=0.9 n$

Figura 4.24: Imagens recuperadas a partir da solução do problema (3.3-3.4) para a imagem Lena com nível de ruido $\sigma_{x}=\sigma_{y}=0.01$ e considerando diferentes valores para o número de amostras $\mathrm{m}$. 


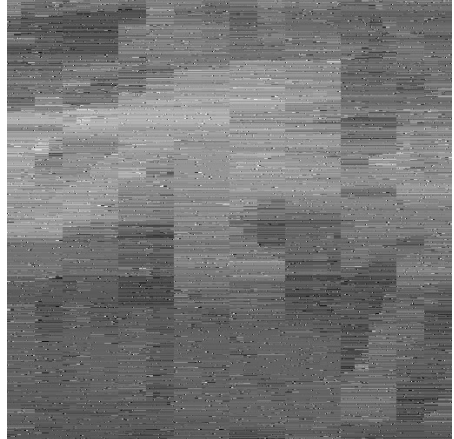

(a) $m=0.1 n$

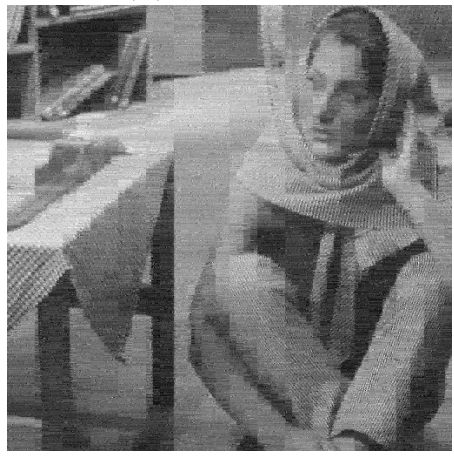

(d) $m=0.4 n$

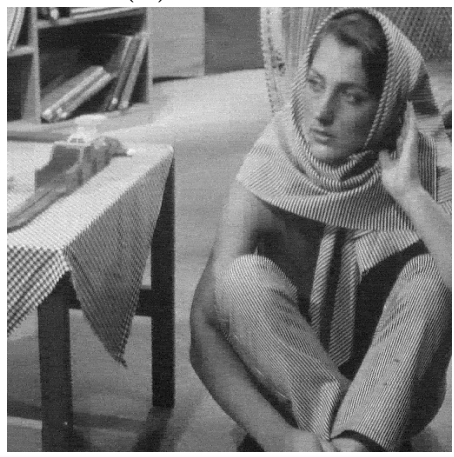

(h) $m=0.7 n$

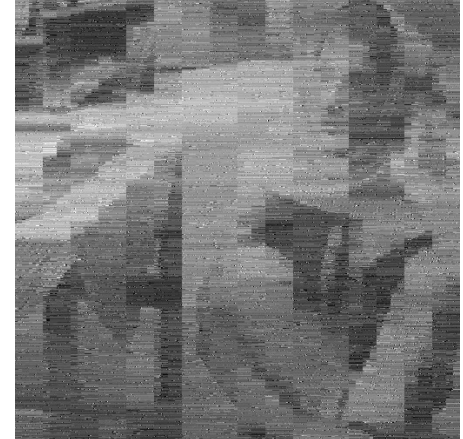

(b) $m=0.2 n$

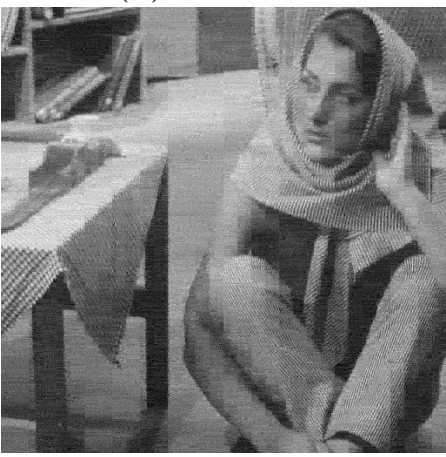

(e) $m=0.5 n$

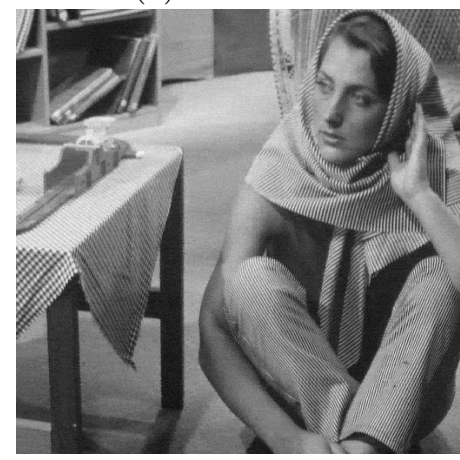

(i) $m=0.8 n$

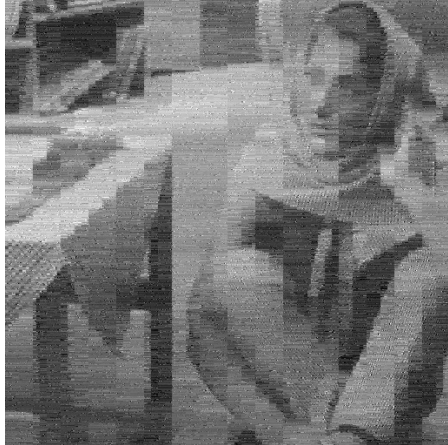

(c) $m=0.3 n$

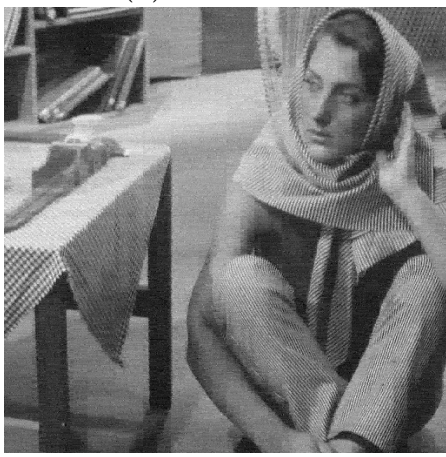

(g) $m=0.6 n$

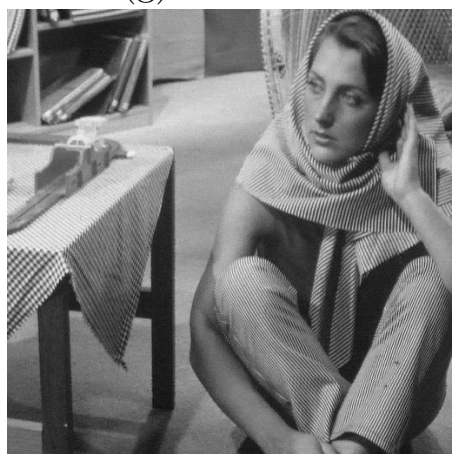

(j) $m=0.9 n$

Figura 4.25: Imagens recuperadas a partir da solução do problema (3.3-3.4) para a imagem Barbara com nível de ruido $\sigma_{x}=\sigma_{y}=0.01$ e considerando diferentes valores para o número de amostras $m$. 


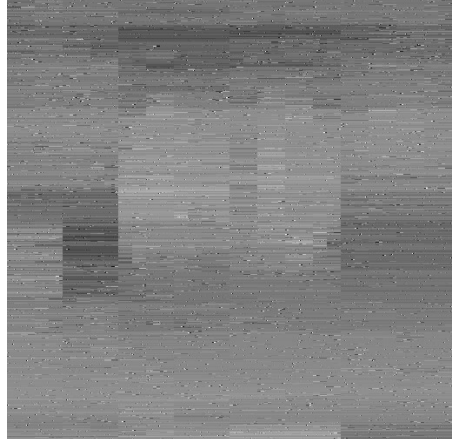

(a) $m=0.1 n$

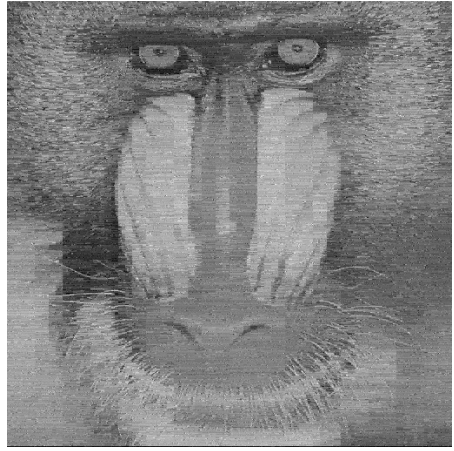

(d) $m=0.4 n$

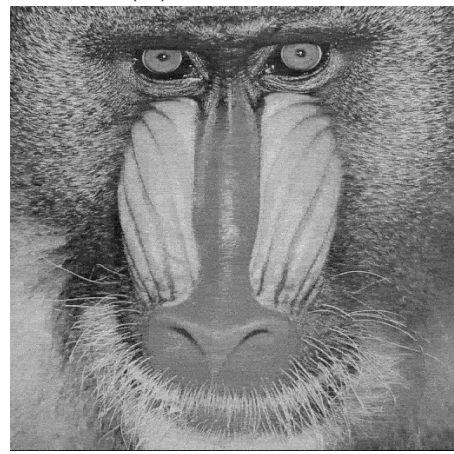

(h) $m=0.7 n$

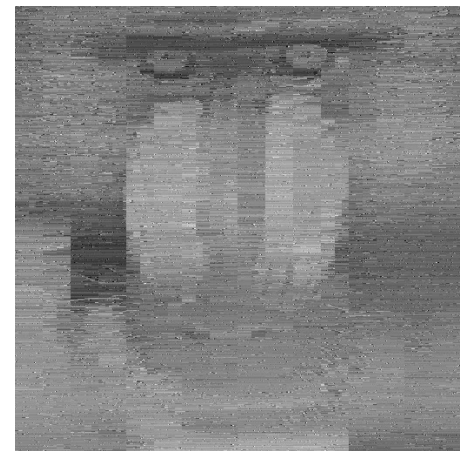

(b) $m=0.2 n$

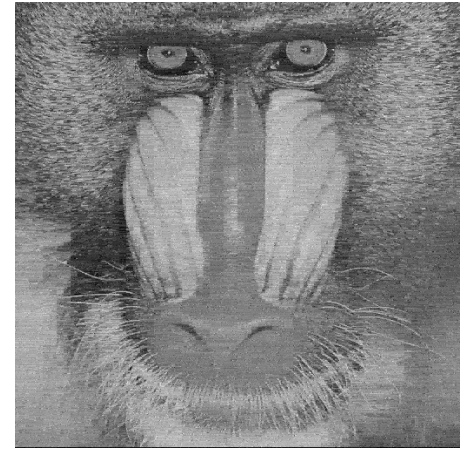

(e) $m=0.5 n$

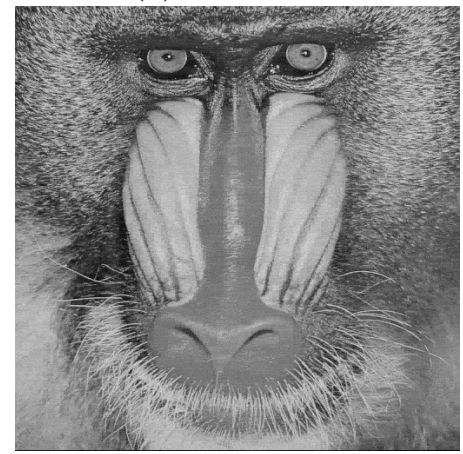

(i) $m=0.8 n$

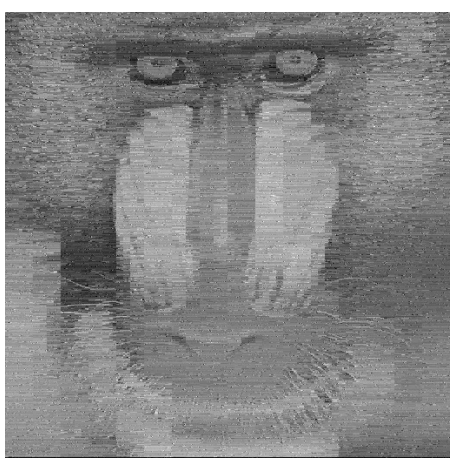

(c) $m=0.3 n$

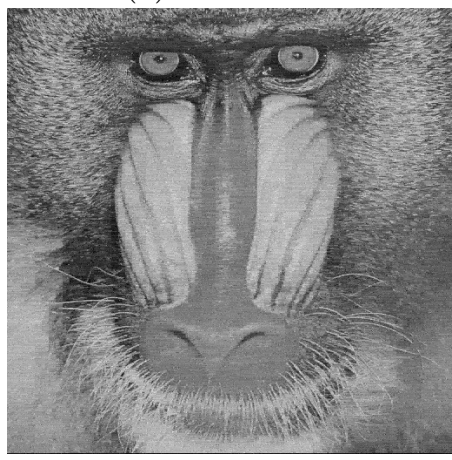

(g) $m=0.6 n$

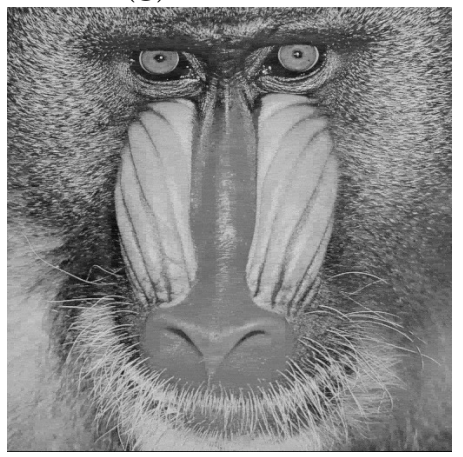

(j) $m=0.9 n$

Figura 4.26: Imagens recuperadas a partir da solução do problema (3.3-3.4) para a imagem Mandrill com nível de ruido $\sigma_{x}=\sigma_{y}=0.01$ e considerando diferentes valores para o número de amostras $m$. 


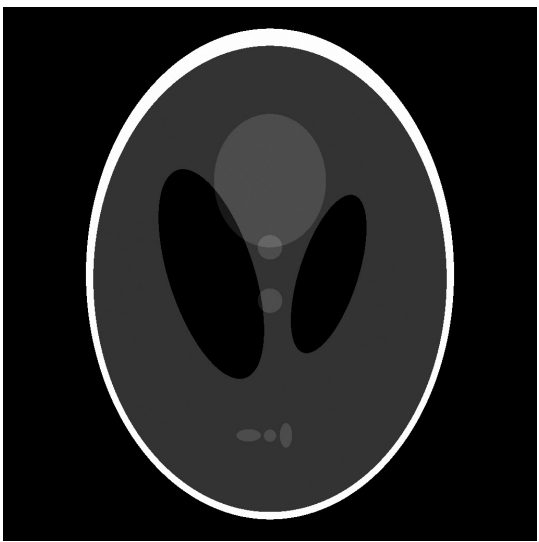

(a)

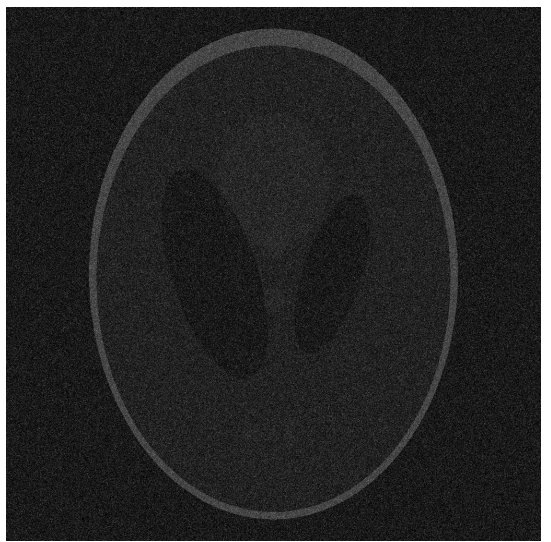

(b)

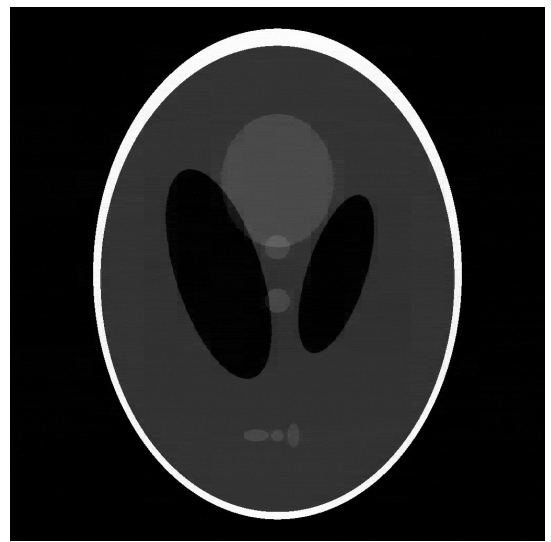

(c)

Figura 4.27: (a) Imagem Phantom de 1,024 $\times 1,024$ pixels com ruido. (b) Imagem que representa o ponto inicial dado por $s_{0}=\Theta^{T}\left(\Theta \Theta^{T}\right)^{-1} y$. (c) Reconstrução via SPG considerando $m=5 k, \sigma_{x}=\sigma_{y}=0.01 e$ $\gamma=0.0011$.

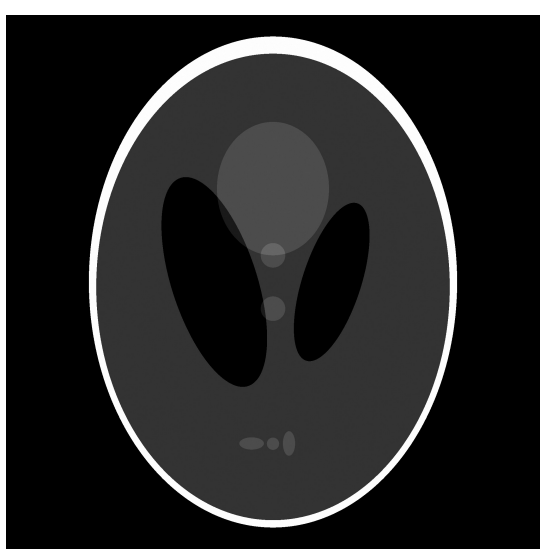

(a)

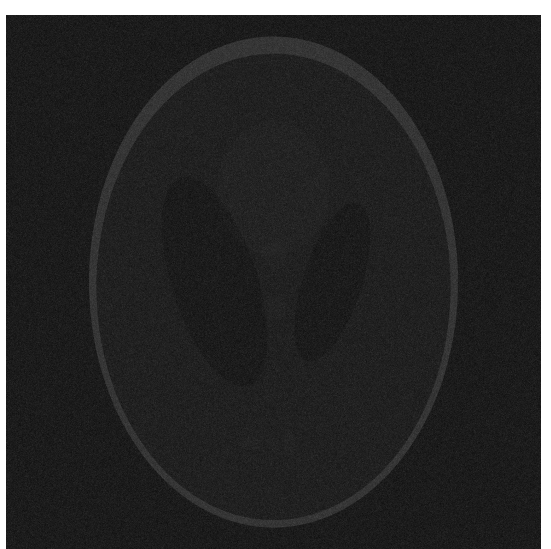

(b)

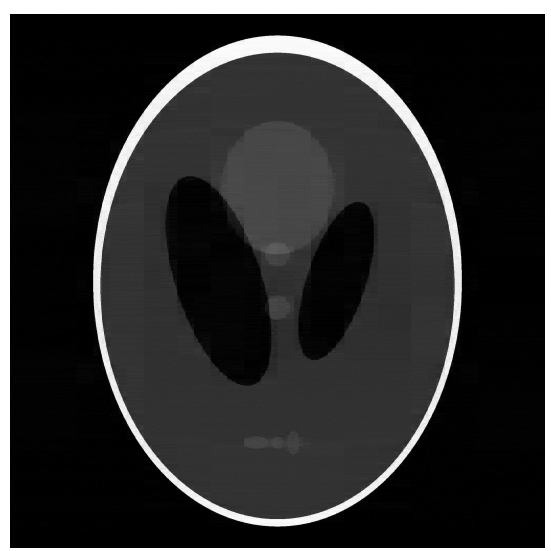

(c)

Figura 4.28: (a) Imagem Phantom de 2,048 $\times 2,048$ pixels com ruido agregado. (b) Imagem que representa o ponto inicial dado por $s_{0}=\Theta^{T}\left(\Theta \Theta^{T}\right)^{-1} y$. (c) Reconstrução via SPG considerando $m=5 k$, $\sigma_{x}=\sigma_{y}=0.01$ e $\gamma=0.0011$. 


\section{Capítulo 5}

\section{Conclusões}

O processo tradicional de compressão de imagens é muito custoso, já que requer a amostragem a armazenamento da imagem original completa para depois descartar os coeficientes menos significativos da sua representação esparsa e assim obter uma versão comprimida do sinal. Compressive Sensing consegue simplificar o processo de aquisição e compressão agrupando-os num só passo.

Neste trabalho estudamos a forma em que o método do Gradiente Espectral Projetado é utilizado no processo de reconstrução de um sinal a partir das amostras. O estudo seguiu de perto a abordagem introduzida em [33]. Com leituras complementares, estudamos conceitos básicos de matrizes de amostragem e matrizes que poderiam se utilizadas como uma base na qual o sinal original tem uma representação esparsa. Com relação às matrizes de amostragem ou medição, nos concentramos nas chamadas Binary Permuted Block Diagonal Matrix [52]. Com relação às matrizes utilizadas como base esparsa, estudamos a transformada discreta da wavelet de Haar [36, 57]. Outras matrizes de medição $[19,24,17,28]$ e matrizes que poderiam ser utilizadas como base esparsa, como por exemplo, a transformada do cosseno discreto [55] e a transformada discreta de Fourier [56] não foram abordadas. De qualquer forma, os conceitos estudados nos forneceram os fundamentos necessários para construir instâncias do problema de otimização relacionado à reconstrução de um sinal amostrado.

No caso em que não há ruido no processo de adquisição das amostras, o problema de reconstrução do sinal original é um problema de programação linear. Resolvemos instâncias deste problema utilizando o solver comercial CPLEX e Algencan. No caso de Algencan, desenvolvemos uma variante que não requer explicitamente da matriz de coeficientes das restrições. Esta versão de Algencan mostrou-se competitiva em problemas de programação linear de grande porte apontando para uma eventual aplicação importante de Algencan em problemas específicos de programação linear.

Quando a amostragem do sinal é afetada por ruido, o SPG pode ser utilizado, como apontado em [33], para resolver uma sequência de problemas que consistem em minimizar uma quadrática convexa sujeita a restrições de não-negatividade nas variáveis. Utilizando a técnica de warmstart, o SPG mostrou-se (como já tinha sido observado na literatura) uma ferramenta eficiente para abordar o problema. Nos nossos experimentos, resolvemos problemas como até $8,338,608$ 
variáveis.

Pelo acima exposto, cumprimos o nosso objetivo de estudar e reproduzir experimentos numéricos relacionados à aplicação do SPG ao problema de Compressive Sensing. Como complemento aos temas abordados no presente trabalho seria interessante estudar os conceitos teóricos de Compressive Sensing que garantem que, sobre certas hipóteses, o sinal original pode ser recuperado com alta probabilidade a partir de um número reduzido de amostras [19, 51]. Seria também interessante fazer um estudo mais aprofundado do trabalho [31] em que o SPG é utilizado para resolver a abordagem Basis Pursuit Denoise do problema de Compressive Sensing. Por útlimo, seria também interessante estudar uma abordagem mais abrangente do problema de $\ell_{1}$-regularization que engloba Compressive Sensing mas tem muitas outras aplicações, como por exemplo, Image Inpainting [2] e o Problema do Google [54]. 


\section{Referências Bibliográficas}

[1] R. Andreani, E. G. Birgin, J. M. Martínez, and J. Yuan, Spectral projected gradient and variable metric methods for optimization with linear inequalities, IMA Journal of Numerical Analysis 25, pp. 221-252, 2005. 29

[2] M. Bertalmio, G. Sapiro, V. Caselles, and C. Ballester, Image inpainting, Proceeding of ACM SIGGRAPH Conference on Computer Graphics, pp. 417-424, 2000. 62

[3] R. Andreani, E. G. Birgin, J. M. Martínez, and M. L. Schuverdt, Augmented Lagrangian methods under the Constant Positive Linear Dependence constraint qualification, Mathematical Programming 111, pp. 5-32, 2008. 30, 33

[4] R. Andreani, E. G. Birgin, J. M. Martínez, and L. M. Schuverdt, On Augmented Lagrangian methods with general lower-level constraints, SIAM Journal on Optimization 18, pp. 1286-1309, 2007. 30, 33

[5] A. S. Bandeira, E. Dobriban, D. G. Mixon, and W. F. Sawin, Certifying the restricted isometry property is hard, Computing Research Repository, 2012. 7

[6] J. Barzilai and J. M. Borwein, Two point step size gradient methods, IMA Journal of Numerical Analysis 8, pp. 141-148, 1988. 27

[7] Z. Ben-Haim, Y. C. Eldar, and M. Elad, Coherence-based performance guarantees for estimating a sparse vector under random noise, IEEE Transactions on Signal Processing 58, pp. 5030-5043, 2010. 8

[8] F. Bergeaud and S. Mallat, Matching pursuit of images, em anais do International Conference on Image Processing, 1995, pp. 53-56. 17

[9] IBM ILOG, CPLEX Optimizer, version 12.1 edition. http://www-01.ibm.com/software/ integration/optimization/cplex-optimizer. 30, 33

[10] E. G. Birgin and J. M. Gentil, Evaluating bound-constrained minimization software, Computational Optimization and Applications, por aparecer (DOI: 10.1007/s10589-012-9466-y). 35

[11] E. G. Birgin and J. M. Martínez, Structured minimal-memory inexact quasi-Newton method and secant preconditioners for Augmented Lagrangian Optimization, Computational Optimization and Applications 39, pp. 1-16, 2008. 34

[12] E. G. Birgin, J. M. Martínez, and M. Raydan, Nonmonotone spectral projected gradient methods on convex sets, SIAM Journal on Optimization 10, pp. 1196-1211, 2000. 3, 26, $27,28,29,30,40$

[13] E. G. Birgin, J. M. Martínez, and M. Raydan, Inexact Spectral Projected Gradient Methods on Convex Sets, IMA Journal of Numerical Analysis 23, pp. 539-559, 2003. 29 
[14] E. G. Birgin, J. M. Martínez, and M. Raydan, Spectral Projected Gradient Methods, em Encyclopedia of Optimization, C. A. Floudas and P. M. Pardalos eds., 2nd ed., Springer US, 2009, pp. 3652-3659. 29

[15] S. Kim, K. Koh, M. Lustig, S. Boyd, and D. Gorinvesky, An interior point method for large-scale $\ell_{1}$-regularized least square, IEEE jornal of selected topics in signal processing, 1, pp. 606-617, 2007. 42

[16] E. G. Birgin, J. M. Martínez, and M. Raydan, Algorithm 813: SPG - software for convexconstrained optimization, ACM Transactions on Mathematical Software 27, pp. 340-349, 2001. 3, 26, 29, 30, 40

[17] E. Candès and J. Romberg, Sparsity and incoherence in compressive sampling, Inverse Problems 23, 969-985, 2007. 5, 16, 61

[18] E. Candès, J. Romberg, and T. Tao, Robust uncertainty principles: exact signal reconstruction from highly incomplete frequency information, IEEE Transactions on Information Theory 52, pp. 489-509, 2006. 1, 9

[19] E. Candès, J. Romberg, and T. Tao, Stable signal recovery from incomplete and inaccurate measurements, Communications on Pure and Applied Mathematics 59, pp. 1207-1223, 2006. $16,61,62$

[20] E. Candès and M. B. Wakin, An introduction to compressive sampling, IEEE Transactions on Signal Processing 25, pp. 21-30, 2008. 5

[21] E. J. Candès, The restricted isometric property ans its implications for compressed sensing, Comptes Rendus Mathematique 346, pp. 589-592, 2008. 6, 7

[22] S. S. Chen, D. L. Donoho, and M. A. Saunders, Atomic decomposition by basis pursuit, SIAM Review 43, pp. 129-159, 2001. 9, 10

[23] Peak signal-to noise ratio, 2012, http://en.wikipedia.org/wiki/Peak_signal_to_noise_ratio. 41

[24] R. Coifman, F. Geshwind, and Y. Meyer, Noiselets, Applied and Computational Harmonic Analysis 10, pp. 27-44, 2001. 16, 61

[25] D. Cores, R. Escalante, M. Gonzalez-Lima, and O. Jimienez, On the use of the Spectral Projected Gradient method for Support Vector Machines, Computational \& Applied Mathematics 28, pp. 327-364, 2009. 27

[26] P. Cupertino de Lima, Wavelets: uma introdução, Matemática Universitária 33, pp. 13-44, 2002. $13,14,15$

[27] S. David and W. Michael, JPEG 2000: Image Compression Fundamentals, Standards and Practice, Kluwer Academic Publishers, Norwell, MA, USA, 2001. 4

[28] T. T. Do, Lu Gan, N. H. Nguyen, and T. D. Tran, Fast and efficient compressive sensing using structurally random matrices, IEEE Transactions on Signal Processing 60, pp. 139-154, 2012. 16, 61

[29] D. L. Donoho and X. Huo, Uncertainty principles and ideal atomic decomposition, IEEE Transactions on Information Theory 47, pp. 2845-2862, 2001. 7 
[30] M. Duarte, M. Davenport, D. Takhar, J. Laska, T. Sun, K. Kelly, and R. Baraniuk, Singlepixel imaging via compressive sampling, IEEE Signal Processing Magazine 25, pp. 83-91, 2008. 1,2

[31] E. van den Berg and M. P. Friedlander, Probing the Pareto frontier for basis pursuit solutions, SIAM Journal on Scientific Computing 2, pp. 890-912, 2008. 10, 27, 62

[32] J. E. Dennis Jr and R. B. Schnabel, Numerical Methods for Unconstrained Optimization and Nonlinear Equations, SIAM, Philadelpia, 1996. 27

[33] M. Figueiredo, R. Niwal, and S. Wright, Gradient projection for sparse reconstruction: Application to compressed sensing and other inverse problems, IEEE Journal of Selected Topics in Signal Processing 1, pp. 586-597, 2007. 3, 10, 11, 18, 27, 40, 41, 61

[34] R. M. Hestenes and E. Stiefel, Methods of Conjugate Gradients for Solving Linear Systems. Journal of Research of the National Bureau of Standards 49, pp. 409-436, 1952. 42

[35] M. R. Garey and D. S. Johnson, Computers and Intractability; A Guide to the Theory of NP-Completeness, W. H. Freeman \& Co., New York, NY, USA, 1990. 8

[36] A. Haar, Zur theoric der orthonormalen funktionen-systeme, Mathematische Annalen 69, pp. 331-371, 1910. 11, 61

[37] F. J. Herrmann and G. Hennenfent, Non-parametric seismic data recovery with curvelet frames, Geophysical Journal International 173, pp. 233-248, 2008. 2

[38] A. Jerri, The Shannon sampling theorem - Its various extensions and applications: A tutorial review, Proceeding of the IEEE 65, pp. 1565-1596, 1977. 1

[39] F. Lampariello, L. Grippo, and S. Lucidi, A nonmonotone line search technique for Newton's method, SIAM Journal on Numerical Analysis 23, pp. 707-716, 1986. 27

[40] Chinh La and M. N. Do, Tree-based orthogonal matching pursuit algorithm for signal reconstruction, em anais do IEEE International Conference on Image Processing, 2006, pp. 1277-1280. 17

[41] M. Lustig, D. Donoho, and J. Pauly, Sparse MRI: The application of compressed sensing for rapid MR imaging, Magnetic Resonance in Medicine 58, pp. 1182-1195, 2007. 2

[42] J. Laska, M. Wakin, M. Duarte, D. Baron, S. Sarvotham, K. Kelly D. Takhar, and R. Baraniuk, An architecture for compressive imaging, em anais do IEEE International Conference on Image Processing, 2006, pp. 1273-1276. 1

[43] S. Mallat, A Wavelet Tour of Signal Processing - The Sparse Way, Academic Press, 3rd edition, 2008. 11

[44] K. M. Miettinen, Nonlinear Multiobjective Optimization, Kluwer Academic Publishers, 1999. 9

[45] J. Nocedal and S. Wright, Numerical Optimization, Springer-Verlag, New York, 2nd ed., 2006. 42

[46] J. A. de Lima, Otimização em Meteorologia: cálculo de perturbações condicionais nãolineares ótimas, Dissertação de Mestrado, Instituto de Matemática e Estatística, Universidade de São Paulo, Brasil, 2011. 27 
[47] P. R. Sampaio, Teoria, métodos e aplicações de otimização multiobjetivo, Dissertação de Mestrado, Instituto de Matemática e Estatística, Universidade de São Paulo, Brasil, 2012. 9

[48] A. Schulz, Compressive Sensing, Novos paradigmas para aquisição e compressão de imagens, Trabalho de Formatura, Escola Politécnica, UFRJ, 2008. 6

[49] T. Serafini, G. Zanghirati, and L. Zanni, Gradient projection methods for quadratic programs and applications in training support vector machines, Optimization Methods 83 Software 20, pp. 347-372, 2005. 27

[50] R. Tibshirani, Regression shrinkage and selection via the Lasso, Journal of the Royal Statistical Society - Series B (Methodological) 58, pp. 267-288, 1996. 10

[51] Y. Tsaig and D. L. Donoho, Compressed sensing, IEEE Transactions Information Theory 52, pp. 1289-1306, 2006. 1, 4, 16, 62

[52] T. Ogawa, Z. He, and M. Haseyama, The simplest measurement matrix for compressed sensing of natural images, em anais do 17th IEEE International Conference on Image Processing, pp. 4301-4304, 2010 3, 16, 17, 61

[53] L. A. Zadeh, Optimality and non-scalar-valued performance criteria, IEEE Transactions on Automatic Control 8, pp. 59-60, 1963.

[54] Yu. Nestreov, Efficiency of coordinate descent methods on huge-scale optimization problems, Optimization Online (http://www.optimization-online.org/DB_FILE/2010/01/ 2527.pdf). 62

[55] Discrete cosine transform, 2012, http://en.wikipedia.org/wiki/Discrete_cosine_transform. 61

[56] Discrete Fourier transform, 2012, http://en.wikipedia.org/wiki/Discrete_Fourier_ transform. 61

[57] Discrete wavelet transform, 2012, http://en.wikipedia.org/wiki/Discrete_wavelet_ transform. 61

[58] http://decsai.ugr.es/cvg/dbimagenes/index.php. 35, 46

[59] http://octave.sourceforge.net/image/function/imresize.html. 35

[60] http://www.gnu.org/software/octave/. 35

[61] http://octave.sourceforge.net/image/function/phantom.html. 35 